\title{
THE SUMMER MEETING IN UNIVERSITY PARK
}

The sixty-second Summer Meeting of the American Mathematical Society was held at Pennsylvania State University, University Park, Pennsylvania, on Tuesday through Friday, August 27-30, 1957, in conjunction with meetings of the Mathematical Association of America, the Society for Industrial and Applied Mathematics, and $\mathrm{Pi} \mathrm{Mu}$ Epsilon. The registration was 649 including 493 members of the Society.

Professor N. E. Steenrod of Princeton University delivered the Colloquium lectures, entitled Cohomology operations, and obstructions to extending continuous functions on Tuesday at 2:00 P.M. and Wednesday, Thursday, and Friday at 9:00 A.M. Presiding officers were Professors Salomon Bochner, Samuel Eilenberg, Leo Zippin, and G. W. Whitehead.

By invitation of the Committee to Select Hour Speakers for Annual and Summer Meetings, Professor A. P. Calderòn of the Massachusetts Institute of Technology addressed the Society on Singular integrals and differential equations on Wednesday at 11:00 A.M., and Professor M. A. Rosenlicht of Northwestern University delivered an hour address, entitled Rationality questions on algebraic groups, on Thursday at 10:30 A.M. Presiding officers were Professors Tibor Radó and A. M. Gleason, respectively.

There were fifteen sessions for contributed papers. The presiding officers were Professors R. D. Anderson, J. H. Barrett, H. P. Evans, Orrin Frink, Jr., M. R. Hestenes, G. W. Mackey, N. H. McCoy, H. T. Muhly, E. D. Nering, Dr. W. H. Pell, Professor J. H. Roberts, Dr. R. L. San Soucie, Professors E. V. Schenkman, I. M. Sheffer, and Dr. W. C. Taylor.

A reception in the Hetzel Union Building was held on Wednesday afternoon. There was a chicken barbecue on Thursday evening in Horticulture Woods.

A banquet was held in the Hetzel Union Building on Wednesday evening. Professor H. B. Curry acted as toastmaster. The speakers were Dean Ben Euwema, Professor Orrin Frink, and President Eric A. Walker of Pennsylvania State University, Professors E. J. McShane, G. B. Price, J. S. Frame, and Dr. T. H. Southard. A resolution of thanks to Pennsylvania State University and to the Arrangements Committee, of which Professor Evan Johnson, Jr., was the chairman, was presented by Professor W. L. Hart.

The Council met on Tuesday afternoon and evening, August 27, 1957. 
The Secretary announced the election of the following eighty-nine persons to ordinary membership in the Society:

Mrs. Muriel S. Alexander, Rumson, New Jersey;

Dr. J. S. Aronofsky, Magnolia Petroleum Co., Dallas, Texas;

Mr. Changmo Band, Choongang High School, Seoul, Korea;

Mr. Milton Bauman, International Business Machines Corporation, Philadelphia, Pennsylvania;

Mr. H. M. Beck, Naval Research Laboratory, Washington, D. C.;

Mr. L. A. Blount, Naval Air Development Center, Johnsville, Pennsylvania;

Mr. J. E. Bogar, Haller, Raymond and Brown, Inc., State College, Pennsylvania;

Dr. A. M. Bomberault, Institute of Mathematical Sciences, New York University;

Mr. J. L. Borden, University of California, Los Angeles;

Mr. G. L. Borman, University of Wisconsin, Milwaukee;

Mr. J. E. Boyd, Western Electric Company, Kearny, New Jersey;

Mr. T. S. Bright, The Girdler Company, Orlando, Florida;

1st Lt. T. A. Brown, U. S. Air Force, Wright-Patterson Air Force Base, Ohio;

Mr. R. T. Carty, Service Bureau Corporation, International Business Machines Corp., St. Louis, Missouri;

Miss Inge F. Christensen, Catholic University of America;

Dr. John Christopher, Burroughs Corporation, Pasadena, California;

Mr. J. E. Conboy, Providence, Rhode Island;

Mr. A. W. Coutris, Columbia University;

Professor William Craig, Pennsylvania State University;

Mr. J. W. Creely, Wallerstein Company, Staten Island, New York;

Mr. R. A. DeSantis, Marquardt Aircraft Co., Van Nuys, California;

Mr. Alfred Descloux, Bell Telephone Laboratories, New York, New York;

Mr. John W. Drane, University of Florida;

Mr. Ronald E. Drummond, Rutgers University;

Captain R. C. Eckerlin, U. S. Air Force, Kirtland Air Force Base, New Mexico;

Mr. S. E. Elliott, Phillips Petroleum Company, Bartlesville, Oklahoma;

Mr. F. D. Farley, University of Michigan;

Mr. L. A. Forrest, Vickers Incorporated, Detroit, Michigan;

Mrs. Amy E. A. Griffin, U. S. Naval Ordnance Test Station, China Lake, California;

Miss Kathryn B. Hanchon, General Electric Company, Cincinnati, Ohio;

Mr. Hershel I. Harrison, Wayne State University;

Mr. Paul Heit, Prudential Insurance Company of America, Newark, New Jersey;

Dr. Jack Heller, Institute of Mathematical Sciences, New York University;

Mr. Marvin H. Hewitt, Glenn L. Martin Company, Middle River, Maryland;

Mr. Ronald E. Hill, Berkeley, Ċalifornia;

Dr. R. R. Hunziker, Reed Research Inc., Washington, D. C.;

Mrs. Louise F. Hutchinson, Bryn Mawr College;

Mr. P. M. Kahn, University of Michigan;

Mr. Shmuel Kantorovitz, Ministry of Defense, Tel-Aviv, Israel;

Professor J. F. Keller, S.J., Loyola University;

Mr. Stanley Kertzner, Brown University;

Mr. G. F. Kottler, General Astronautics Corporation, New York, New York:

Mr. Andrew Kraus, National Bureau of Standards, Boulder, Colorado;

Mr. Ora K. Krienke, Jr., Seattle Pacific College;

Mr. R. H. Kupperman, New York University; 
Dr. Herbert Kurss, New York University;

Professor W. M. Laird, University of Pittsburgh;

Professor Lester H. Lange, Valparaiso University;

Mr. H. S. Lauson, University of California, Los Alamos;

Dr. W. S. McCulloch, Massachusetts Institute of Technology;

Mr. W. G. Madison, Avco Manufacturing Corporation, Boston, Massachusetts;

Professor Felina G. Mapa, University of the Philippines;

Miss Bertha W. Mather, Pennsylvania State University;

Mr. George J. Minty, University of Michigan;

Mr. R. A. Moore, University of Texas;

Miss Vivian Morgan, North American Aviation, Canoga Park, California;

Mr. H. W. Nace, Lawrence Institute of Technology;

Mr. Samuel Orlinick, Scientific Library Service, New York, New York;

Professor C. D. Oviatt, Tarkio College;

Professor C. K. Pahk, Kyungpook University, Taegu, Korea;

Mr. Samuel Park, University of Pittsburgh;

Mr. G. B. Parrish, Office of Ordnance Research, Durham, North Carolina;

Professor J. M. Perry, Clarkson College of Technology;

Miss Carol B. Peters, Hoover Microwave Company, College Park, Maryland;

Mr. Richard Possamai, Newark College of Engineering;

Mr. A. F. Quesada, Baird Atomic, Inc., Cambridge, Massachusetts;

Mr. W. C. Rheinbolt, National Bureau of Standards, Washington, D. C.;

Professor David Rosenblatt, American University;

Dr. Joan R. Rosenblatt, National Bureau of Standards, Washington, D. C.;

Mr. R. S. Rosenzweig, Allstate Insurance Company, Skokie, Illinois;

Professor J. E. Rowe, University of Michigan;

Mr. Dominick A. Russo, Sperry Gyroscope Co., Great Neck, New York;

Professor D. A. Rux, Wisconsin State College, Oshkosh;

Mr. Peter F. Stebe, Queens College;

Mr. E. C. Throndsen, General Electric Company, New York, New York;

Miss Eugenia I. Trapp, International Business Machines Corporation, Houston, Texas;

Mr. William F. Trench, Philco Corporation, Philadelphia, Pennsylvania;

Mr. T. E. Truan, Crucible Steel Company of America, Pittsburgh, Pennsylvania;

Mr. L. H. Turner, Purdue University;

Mr. W. E. Watts, Reynolds Metals Company, Listerhill, Alabama;

Mr. David M. Waxman, Nuclear Development Corporation of America, White Plains, New York;

Mr. M. S. Weinstein, Employees' Retirement System, Albany, New York;

Miss Miriam Weissner, AEC Computing Facility, New York University;

Mr. C. S. West, Jr., International Fidelity Insurance Company, Dallas, Texas;

Miss Edith S. Windsor, New York University;

Professor Izaak Wirszup, University of Chicago;

Miss Monica J. Wyzalek, University of Illinois;

Mr. Floyd E. Young, Western Life Insurance Company, Helene, Montana;

Mr. J. M. Zimmerman, Rocketdyne, North American Aviation Corporation, Canoga Park, California.

It was reported that the following eight persons had been elected to membership on nomination of institutional members as indicated: 
Montana State University: Mr. J. A. Peterson.

University of North Carolina: Mr. L. L. Bumgarner, Mr. J. H. Keller, Mr. J. P. McAllister, Mr. M. B. Smith, Jr., Mr. D. P. Stanford.

Purdue University: Mr. R. E. Dowds.

University of Rochester: Mr. G. C. Branche.

The Secretary reported that the following had been admitted to the Society in accordance with reciprocity agreements with various mathematical organizations: Deutsche Mathematiker-Vereinigung: Dr. Detlef Laugwitz, Dr. Hanfried Lenz; Société Mathématique de France: Professor André Lentin, Mr. Jean Riss, Mr. Aimé Sestier, Mr. Weishu Shih; London Mathematical Society: Professor Geoffrey Bosson, Dr. Ronald Harrop, Professor Patrick B. Kennedy; Schweizerische Mathematische Gesellschaft: Dr. Heinz T. Renggli.

The following appointments by the President were reported: as a Committee to Nominate Officers and Members of the Council for 1958: C. B. Allendoerfer, Chairman, R. P. Boas, G. W. Mackey, S. Ulam, A. D. Wallace; as an arrangements Committee for the November, 1958, meeting in Coral Gables, Florida: W. L. Strother, C. E. Capel, Georgia K. Del Franco, J. H. Roberts; as a Committee to Recommend Gibbs Lecturers for 1958 and 1959: S. Ulam, Chairman, Lipman Bers, Wilfred Kaplan; as a Committee to Award the Bôcher Prize in 1958: S. Bochner, Chairman, J. L. Doob, D. V. Widder; as a Program Committee for a Symposium on Combinatorial Designs and Analysis: Marshall Hall, Chairman, Richard Bellman, S. A. Schelkunoff, Claude Shannon, and C. B. Tompkins; as a Committee to Recommend Recipients of National Science Foundation Travel Grants to the International Congress of Mathematicians in Edinburgh, Scotland, 1958: R. L. Wilder, Chairman, E. Artin, G. W. Mackey, S. S. Chern, N. Levinson, K. O. Friedrichs, O. Zarisky; as a member of the Committee to Select Hour Speakers for Western Sectional Meetings: R. C. Buck (until December 31, 1958, to fill out the unexpired term of A. C. Schaeffer); as a delegate to the inauguration of Richard Palmer as the tenth President of Morningside College, Dr. Marjorie H. Beaty.

The Secretary reported that the following had accepted invitations to deliver hour addresses: A. H. Clifford at the University of Miami, November 1957; W. H. Gottschalk, Washington, D. C., October 1957; Leo Sario at Corvallis, Oregon, June 1958; Daniel Zelinsky at Columbia, Missouri, November 1957.

The following dates and places of meetings were set: April 25 and 26, 1958 at Columbia University; June 20,1958, at the Oregon State College; and August-September 1959 at the University of Utah. 
The Council voted that beginning with 1958 , abstracts shall be published in the Notices instead of in the Bulletin. The space saved in the Bulletin will be used for short announcements of the sort that have appeared in the Proceedings of the National Academy of Sciences. Further details concerning these new arrangements will appear shortly.

A business meeting of the Society was held at 10:00 A.M., August 28, with Vice President Bochner presiding. The Secretary reported briefly on the affairs of the Society.

The following amendments to the by-laws were adopted:

Article VII, Section 1. The last sentence was amended to read: All terms of office shall begin on January 1 and terminate of December 31 with the exception that the officials specified in Articles I, II, III, IV, and V (excepting the President Elect and Ex-President) shall continue to serve until their successors have been duly elected and qualified.

Article VII, Section 2. The article was amended to read: The President Elect, the Vice Presidents, the Secretary, the Associate Secretaries, the Treasurer, the Trustees, the members of the Publications Committees, and the members-at-large of the Council shall be elected by written ballot. An official ballot shall be sent to each member of the Society by the Secretary on or before October 10, and such ballots if returned to the Secretary in envelopes bearing the name of the voter and received within 30 days shall be counted. Each ballot shall contain one or more names proposed by the Council for each office to be filled, with blank spaces in which the voter may substitute other names. A plurality of all votes cast shall be necessary for election. In case of failure to secure a plurality for any office, the Council shall choose by written ballot among the members having the highest number of votes.

Article VII, Section 4. The first sentence was amended to read: On or before January 15, the Secretary shall send to all members of the Council for a mail vote a ballot containing two names for each place to be filled on the Executive Committee.

A new section was added to Article VII as follows:

Section 9. In case any officer dies or declines to serve between the time of election and the time at which he was to assume office, the vacancy shall be filled in the same manner as if he had served one day of his term.

Article IX, Section 5. This article was amended to read: The minimum annual dues of a corporate member shall be one thousand dollars. 
Article X, Section 1. The first sentence was amended to read: The Annual Meeting of the Society shall be held between the fifteenth of December and the tenth of February next following.

Article X, Section 4. The article was amended to read: The Board of Trustees shall hold at least one meeting in each calendar year. Meetings of the Board of Trustees may be called by the President, the Treasurer, or the Secretary of the Society upon three days' notice of such meetings mailed to the last known post office address of each Trustee. The Secretary of the Society shall call a meeting upon the receipt of a written request of two of the Trustees. Meetings may also be held by common consent of all the Trustees.

The abstracts of the papers follow. Those having the letter " $t$ " following the abstract number were read by title. Where a paper, presented in person, has more than one author, the symbol ( $p$ ) follows the name of the author who presented it. Mr. Appling was introduced by Professor H. S. Wall, Mr. Crowe by Dr. J. S. Griffin, Mr. Ito by Professor Hidehiko Yamabe, and Mr. Sublette by Professor Seymour Sherman.

\section{Algebra AND Theory of Numbers}

\section{8t. S. B. Akers, Jr.: On a theory of Boolean functions.}

Given a Boolean function, $F\left(x_{1}, x_{2}, \cdots, x_{m}\right)$, the partial derivative of $F$ with respect to a basic variable, $x_{i},(1 \leqq i \leqq m)$, is denoted by $F_{x_{i}}$ and defined as $F_{x_{i}}$ $=F\left(x_{1}, \cdots, x_{i}, \cdots, x_{m}\right) \oplus F\left(x_{1}, \cdots, x_{i}^{\prime}, \cdots, x_{m}\right)$ where $\oplus$ denotes exclusive disjunction. It follows that a necessary and sufficient condition that $F$ be a dependent function of $x_{i}$ is that $F_{x_{i}} \neq 0$ where 0 is the null element. This notion of a Boolean derivative is applied to such concepts as series expansions, integration, functional decomposability, and functional invariance. Many concepts of ordinary function theory such as Jacobians, constants of integration, Taylor and Maclaurin series, chain rule of partial differentiation, etc., are found to have counterparts in the resulting theorems. The application of the results to switching circuit analysis and synthesis is discussed. In particular, given $F\left(x_{1}, x_{2}, \cdots, x_{m} ; y_{1}, y_{2}, \cdots, y_{n}\right)$ and $G\left(y_{1}, y_{2}, \cdots, y_{n}\right)$ a necessary and sufficient condition that $F$ be a dependent function of $G$ is derived in terms of their partial derivatives. An existence theorem is also proved-i.e., given $F$, under what conditions does there exist a function $G$ such that $F$ is a dependent function of $G$ ? (Received May 23, 1957.)

\section{H. L. Alder: A generalization of the Euler $\phi$-function.}

The function $\phi(n, m)$ with $m \geqq n$ is defined as the number of ordered pairs $\langle x, y\rangle$ for which $x+y=m, 1 \leqq x \leqq n$, and $x$ and $y$ both relatively prime to $n$. Since, for $m=n$, this function obviously reduces to the Euler $\phi$-function, $\phi(n, m)$ is a generalization of the latter. A simple formula for the calculation of $\phi(n, m)$ is given. (Received July 10, 1956.) 


\section{D. R. Anderson: On the estimation of the Kloosterman sum.}

This paper deals with the estimation by analytic methods of the magnitude of the Kloosterman sum $K\left(m_{1}, m_{2} ; n\right)$ in the case where $n$ is a prime $p>2$. This is defined by the following relation: $K\left(m_{1}, m_{2} ; n\right)=\sum_{1 \leqq h \leqq n ;(h, n)-1} \exp \left[(2 \pi i / p)\left(m_{1} h+m_{2} \bar{h}\right)\right]$, where $m_{1}, m_{2}$ and $n$ are positive integers and $h \bar{h} \equiv 1(\bmod p)$. (For basic properties see Acta Math. vol. 49 (1926) pp. 407-464.) The best previous estimate was due to A. Weil. (See Proc. Amer. Acad. Sci. vol. 34 (1938) pp. 204-207.) This estimate is: $\left|K\left(m_{1}, m_{2} ; p\right)\right| \leqq 2 p^{1 / 2}$. The estimate of the present paper results as follows. By way of a method similar to that used by Mordell in obtaining the formula for the Gauss sum, $K\left(m_{1}, m_{2} ; p\right)$ is expressed in terms of several contour integrals. The latter integrals are re-expressed in the form from which the estimate is made through analytic continuation and Mellin transform theory. The result obtained is: $\left|K\left(m_{1}, m_{2} ; p\right)\right|$ $\leqq C(\log p)^{2}, C$ being a constant relative to all variables. (Received August 28, 1957.)

\section{Rafael Artzy: Cross-inverse loops.}

A cross-inverse (C.I.) loop is defined as a loop satisfying the identity $x y \cdot x^{\prime}=y$, where $x^{\prime}$ is the right inverse of $x$. Isotopic C.I. loops are isomorphic. A principal isotope of a C.I. loop $G$, determined by $x g \circ f y=x y$, is C.I. if and only if $f x \cdot g y=f g \cdot x y$ for all $x$ and $y$ in $G$. Every autotopism $(U, V, W)$ of a C.I. loop determines uniquely a C.I. principal isotope with $1 U=f, 1 V=g$. The $f$ 's and $g$ 's of all autotopisms of a C.I. loop form a normal subloop of $G$ which is commutative and Moufang. For each C.I. principal isotope of a C.I. loop, $\mathrm{fg}^{-1}$ is in the centre of $G$. (Received July 3, 1957.)

\section{2t. S. K. Berberian: $n \times n$ matrices over an $\mathrm{AW}^{*}$-algebra.}

Theorem: if $A$ is any $\mathrm{AW}^{*}$-algebra, the algebra $A_{n}$ of $n \times n$ matrices over $A$ is an $\mathrm{AW}^{*}$-algebra. (Received July 10, 1957.)

\section{H. E. Campbell: On Whitehead's second lemma.}

An alternative algebra is called nondegenerate if for right multiplications $R_{x}$ and $R_{y}$, trace $\left(R_{x} R_{y}\right)$ is a nondegenerate bilinear form. A direct proof of the second Whitehead lemma, which was discussed by Schafer (Proc. Amer. Math. Soc. vol. 4 (1953) pp. 444-451), for nondegenerate alternative algebras of arbitrary characteristic is obtained by using the Casimir operator defined in the author's On the Casimir operator (submitted to Pacific J. Math.). This implies the Wedderburn principal theorem (Schafer, Bull. Amer. Math. Soc. vol. 55 (1949) pp. 604-614) and simplifies its proof for the class of nondegenerate algebras. (Received July 12,1957.)

\section{4t. Eckford Cohen: Representations of even functions $(\bmod r)$.}

This paper is concerned with the theory of even arithmetical functions of $n(\bmod r)$, defined by $f(n, r)=f((n, r), r), r \geqq 1$ [Proc. Nat. Acad. Sci., vol. 41 (1955) pp. 939944]. The paper is divided into five parts, summarized as follows. (1) The fundamental theorem on even functions $(\bmod r)$ is reformulated in a manner useful for applications; (2) a new proof of the Anderson-Apostol theorem on functions satisfying a Hölder relation is given; (3) an inversion formula for even functions $(\bmod r)$ is proved; (4) completely even functions $(\bmod r)$, defined by $f(n, r)=g((n, r))$, are arithmetically characterized; (5) a special class of functions of the form $\sum_{d \mid r} h(d, r / d)$, $(d, n)=1$, is studied. The theory is illustrated with several arithmetical functions, and numerous identities between various functions are deduced as special cases. The paper 
provides, in fact, a general method for establishing certain types of arithmetical identities. (Received July 8, 1957.)

\section{5t. Eckford Cohen: Trigonometric sums in elementary number theory.}

Let $n, r, s$ be integers, $r>0, s \geqq 0$. Denote by $\sigma_{a}^{*}(n, r)$ the number of $x_{i}, y_{i}(\bmod r)$ such that $n \equiv x_{0} y_{0}+\cdots+x_{s} y_{s}(\bmod r)$. Also, let $\phi_{a}^{*}(n, r)$ denote the number of $x_{i}(\bmod r)$ such that $n \equiv x_{0}+\cdots+x_{s}(\bmod r)$, under the restriction $\left(\left(x_{0}, \cdots, x_{s}\right), r\right)$ $=1$. Finite trigonometric and arithmetical expansions of the functions $\sigma_{a}^{*}(n, r)$ and $\phi_{a}^{*}(n, r)$ are deduced. By applying a special limiting process to the results obtained for $\sigma_{0}^{*}(n, r) / r^{*}$ and $\phi_{0}^{*}(n, r) / r^{*}$, Ramanujan's well-known series expansions for $\sigma_{s}(n) / n^{s}$ and $\phi_{s}(n) / n^{*}(n>0, s>0)$ are shown to result, respectively. (Received August 15, 1957.)

\section{Haskell Cohen: Convexity of the kernel in admissible mobs.}

A mob $S$ is a Hausdorff topological semigroup. An admissible mob is in addition a compact convex subset of a real linear topological space satisfying $x \cdot[\alpha y+(1-\alpha) z]$ $=(x \cdot y)+(1-\alpha)(x \cdot z)$ and $[\alpha y+(1-\alpha) z] \cdot x=\alpha(y \cdot x)+(1-\alpha)(z \cdot x)$ for all $x, y, z \in S$ and all $\alpha$ such that $0 \leqq \alpha \leqq 1$ (where "." is the semigroup operation). The kernel $K$ of a mob is its minimal ideal. The following results are obtained. (i) If the dimension of $S \leqq 2, K$ is convex. (ii) if $\operatorname{dim} S \leqq 3$ and $S$ has a unit, $K$ is convex. In addition examples are given of a three dimensional mob with nonconvex kernel, and of a four dimensional mob with unit having a nonconvex kernel. (Received July 10,1957.)

\section{H. S. Collins: Admissible mobs. I. Preliminary Report.}

A semigroup $S$ is affine if $S$ is a convex subset of a real linear space $E$ and multiplication is, separately, an affine mapping of $S$ into $S$. $S$ is an admissible mob if, in addition, $E$ is a Hausdorff linear topological space, $S$ is compact, and multiplication is continuous. In this paper, all finite dimensional affine semigroups with unit and zero are characterized as matrix semigroups; more concrete characterizations are given for one-dimensional affine semigroups with unit, for two-dimensional admissible mobs with unit and no zero, and for any two-dimensional disc with unit and with zero at its center. (Received July 10, 1957.)

\section{8t. R. P. Dilworth: A representation theorem for universal alge-} bras.

Let $\mathfrak{A}$ denote a universal algebraic system. If $\left\{A_{\sigma} \mid \sigma \in \Sigma\right\}$ is a collection of realizations of this system, let $\pi A_{\sigma}$ denote the Cartesian product of algebras, that is, the set of functions $f$ on $\Sigma$ such that $f(\sigma) \in A_{\sigma}$. Operations and relations in $\pi A_{\sigma}$ are defined componentwise. An algebra $A$ is said to be the direct union of the algebras $A_{\sigma}$ if there exists a one-to-one mapping $\phi$ from $A$ into $\pi A_{\sigma}$ such that (1) the operations and relations in $A$ are preserved, and (2) if $\sigma_{1}, \cdots, \sigma_{n}$ is a finite subset of $\Sigma$ and $a_{1}, \cdots, a_{n}$ are elements of $A \sigma_{1}, \cdots, A \sigma_{n}$ respectively, then there exists $a \in A$ such that $\phi\left(a, \sigma_{i}\right)$ $=a_{i}$ for $i=1, \cdots, n$. $A$ is completely indecomposable if whenever $A$ is a direct union of algebras $A_{\sigma}$, the mapping $\phi(a, \sigma)$ is an isomorphism for some $\sigma \in \Sigma$. It is proved that every algebra is a direct union of completely indecomposable algebras. Applications to lattice theory and group theory are given. (Received July 11, 1957.)

629. Philip Dwinger: Completeness of quotient algebras of Boolean algebras.

Let $A$ be a Boolean algebra and $I$ an ideal in $A$. If $X \subset A$, let $\kappa(X)$ denote the 
power of $X$ and $\delta(X)$ the least cardinal number $\rho$, such that the power of every disjointed subset of $X$ is at most $\rho$. $A$ is $\alpha$-complete, $\alpha$ a cardinal number, if for every $X \subset A, \kappa(X) \leqq \alpha, \sum X$ exists. $I$ is $\alpha$-complete if for every $X \subset I, \kappa(X) \leqq \alpha, \sum X$ exists and $\in I$. Tarski and Smith (Trans. Amer. Math. Soc. vol. 84 (1957) pp. 230-257) have proved that if (i) $A$ is $\beta$-complete, $\beta$ transfinite, (ii) $I$ is $\alpha$-complete for every $\alpha<\beta$, (iii) $\delta(A / I) \leqq \beta$, then $A / I$ is complete. In the present paper it is proved, that if one replaces (iii) by (iii) ${ }^{\prime}: \delta(A / I)>\beta$ and adds the condition (iv) $I$ dense and $\sum X \in A-I$ if $X \subset I$ and $\kappa(X) \geqq \beta$, then $A / I$ is not $\beta$-complete. This result is a corollary of a more general theorem which is proved for lattices satisfying a weak infinite distributive law. Another result is the following: if $A$ is $\sigma$-complete ( $A$ not finite), $I$ dense and $\sum X \in A-I$ if $X \subset I, X$ not finite, then $A / I$ is not $\sigma$-complete and there does not exist a nontrivial finitely additive measure $\mu$ on $A$, such that $I=\{x \mid x \in$ $A, \mu(x)=0\}$. (Received June 17, 1957.)

\section{0t. Murray Gerstenhaber: Nilalgebras and linear varieties of nil- potent matrices I.}

Let $A$ be a commutative but not necessarily associative algebra of characteristic zero; $A$ may be of infinite dimension. If there exists an integer $t$ such that the $t$ th power of every $a$ in $A$ in every association is zero, then the linear transformation $R_{a}: x \rightarrow x a$ is nilpotent for all $a$ in $A$. If $A$ is a commutative and power associative algebra of characteristic zero but not necessarily finite dimension, then a nilpotent implies $R_{a}$ nilpotent. (Received May 13, 1957.)

\section{Murray Gerstenhaber ( $\mathrm{p}$ ) and S. A. Hoffman: Nilalgebras} and linear varieties of nilpotent matrices. II.

Let $V$ be a linear variety of nilpotent matrices of dimension $k$ over the complex numbers. Then $k \leqq n(n-1) / 2$, and if $k=n(n-1) / 2$ then there exists a nonsingular matrix $X$ such that $X V X^{-1}$ is in fact the set of all triangular matrices. Since for $n=2,3$ one has $n=n(n-1) / 2$ it is a corollary that if $A$ is an algebra (not necessarily associative) of dimension 2 or 3 over the complex numbers in which $R_{a}$ is nilpotent for all $a$ in $A$, then $A$ is nilpotent. (Received May 13, 1957.)

632t. Emil Grosswald, Anne Calloway and Jean Calloway: The proper representation of integers as sums of 3 squares.

The equation $\left(^{*}\right) n=u^{2}+v^{2}+w^{2}$ has integral solutions, unless $n=4^{a} \cdot n_{1}, 4 \nmid n_{1}$, $n_{1} \equiv 7(\bmod 8)$; for some $n$, however, all such solutions satisfy $u \cdot v \cdot w \cdot=0$. Any integer $n$, for which $\left(^{*}\right)$ has some integral solution with $u \cdot v \cdot w \neq 0$, is said to be properly represented by $(*)$. The problem arises to characterize the integers $n$ with proper representations $\left(^{*}\right)$ and to find the number $N(x)$ of such integers $n \leqq x$. Theorem: The integer $n=4^{a} \cdot n_{1}$ has a proper representation $\left({ }^{*}\right)$, unless either $n_{1} \equiv 7(\bmod 8)$, or else $n_{1} \in S$, where $S$ is a certain finite set of $m$ integers. Furthermore, $N(x)=5 x / 6$ $-(\log 4)^{-1} \sum \log \left(x / b_{i}\right)+R$, the sum being extended over all $b_{i} \in S$ and where $-(m-7 / 24) \leqq R \leqq R_{M}=(\log 4)^{-1} \log (x / 7)+7 / 24$. Conjecture: $S=\{1,2,5,10,13,25$, $37,58,85,130\}, m=10$; hence, $N(x)=5 x / 6-10(\log 4)^{-1} \log x+a+R, a=(\log 4)^{-1}$ $\cdot \sum \log b_{i}=19,7 \ldots$ and $-233 / 24 \leqq R \leqq R_{M}$. Besides classical results (Landau, Hurwitz), the proof is based mainly on Bateman's formula for the number of representations of an integer as a sum of three squares (Trans. Amer. Math. Soc. vol. 71 (1951) pp. 70-101), combined with the Siegel-Tatuzawa estimate of the class-number (Jap. J. Math. vol. 21 (1951) pp. 163-178). These permit to show that for sufficiently 
large $n_{1}$, the number of representations of $n$ by three squares exceeds the (properly counted) number of representations by two squares and the conclusion follows. (Received June 11, 1957.)

633t. D. K. Harrison: Partial cohomology of modules. I Abelian groups. Preliminary report.

Satellites and homology of a functor are taken, not with respect to all exact sequences, but only with respect to special sequences. If a sequence splits, it would be foolish to use only that it is exact. Similarly, if an exact sequence is "tight," this paper attempts to utilize that "tightness" with a theory aimed explicitly at that type of sequence. Several types of sequences are shown to be such that the main theorems of homological algebra apply with exact sequences replaced by sequences of their type. For abelian groups we consider in this sense the cohomology of pure subgroups. The pure projective groups are the direct sums of cyclic groups. The pure injective groups are the sums of a divisible group with a direct summand of a product of finite cyclic groups. These concepts can be described in terms of the degree of completeness of the uniformity of the group $G$ defined by the multiples $n G$. It is proved using these methods that: an abelian group can be made into a compact topological group if and only if it is algebraically isomorphic to a product of $p$-adic integers, finite cyclic groups, the reals, and groups of type $Z\left(p^{\infty}\right)$, where the number of copies of the reals exceeds or is equal to the number of $Z\left(p^{\infty}\right)$ for any $p$; if $A$ and $B$ are torsion groups, $A \otimes B$ is a direct sum of cyclic groups, and $\operatorname{Hom}(A, C)$ is a direct summand of a product of finite cyclic groups; and $H$ is a pure subgroup of $G$ if and only if $0 \rightarrow H \otimes A \rightarrow G \otimes A$ is exact for all $A$. (Received July 11, 1957).

\section{Juris Hartmanis: $A$ note on the lattice of topologies.}

The collection of all topologies on a fixed set $S$ forms a complete point lattice under its natural ordering, i.e. $R_{1} \leqq R_{2}$ if every set closed under $R_{1}$ is also closed under $R_{2}$. It is shown that the lattice of all topologies has only trivial homomorphisms if $S$ consists of more than two elements. On the other hand, this is not true for the lattice of all $T_{1}$ topologies on $S$ and the construction of all homomorphisms is given in this case. It is further shown that the group of automorphisms for the lattice of topologies is (a) isomorphic to a symmetric group if $S$ is infinite, or consists of one or two elements, (b) isomorphic to the direct product of a symmetric group with the two element group otherwise. Finally it is observed that the lattice of topologies is complemented if and only if it is finite. (Received July 8, 1957.)

635t. J. H. Hodges: Scalar polynomial equations for matrices over a finite field.

Let $G F(q)$ denote the finite field of order $q=p^{n}$. If $E=E(x)$ is a monic polynomial over $G F(q)$, let $N(E, m)$ denote the number of matrices $\theta$ of order $m$ over $G F(q)$ such that $E(\theta)=0$. In this paper, the classical theory of the scalar polynomial equation for matrices over a general field is combined with a theorem of L. E. Dickson (Linear Groups, Leipzig, 1901, p. 235) on commutativity of certain matrices over $G F(q)$ to give an explicit formula for $N(E, m)$. The formula is illustrated for the case $E(x)=x^{3}-1$. (Received July 11, 1957.)

\section{6t. Nathan Jacobson and L. J. Paige: On Jordan algebras with two generators.}

An associative algebra $\mathfrak{A}$ over a field of characteristic $\neq 2$ with multiplication 
composition $(X)$ defines a Jordan algebra $\mathfrak{P}^{+}$relative to the Jordan multiplication $a b=(a \times b+b \times a) / 2$. A Jordan algebra is called special if it is isomorphic to a subalgebra of an algebra $\mathfrak{A}^{+}, \mathfrak{A}$ associative. A. I. Shirshov (On special J-rings, Mat. Sbornik vol. 38 (1956)) proved that the free Jordan algebra with two generators is special. The authors give a comparatively simple alternative proof of this result and at the same time determine a simple basis for the algebra. Briefly, a basis for the free special Jordan algebra $\Im_{0}$ (the subalgebra relative to Jordan multiplication generated by two generators in a free associative algebra $F$ ) is defined inductively using Jordan multiplication. Imitating this procedure in the free Jordan algebra $\Im$ with two generators, a set $S$ in $1-1$ correspondence with the basis in $\Im_{0}$ is defined. To prove that $S$ is a basis for $\Im$, three variable identities of independent interest are used. The results on the basis of $\Im_{0}$ are applicable to Jordan algebras of characteristic 2 . Although it is known that any Jordan algebra (of characteristic $\neq 2$ ) with two generators is special, the corresponding result fails for characteristic two even for one generator. (Received June 24, 1957.)

\section{7t. Harry Kesten: Symmetric random walk on groups.}

Let $G$ be a group generated by elements $a_{1}, \cdots, a_{h}$. Consider a random walk on $G$ in which a step consists of multiplication on the right by one of the generators $a_{1}, \cdots, a_{h}$ or its inverse, each possibility having the same probability $(2 h)^{-1}$. This random walk defines a Markov chain, the transition probability from $g_{1}$ to $g_{2}\left(g_{1}, g_{2} \in G\right)$ being the probability that $g_{2}$ is reached in one step from $g_{1}$. Since $G$ is countable this Markov chain can be represented by its matrix $M$ of transition probabilities. The following result is proved. The group $G$ is a free group with independent generators $a_{1}, \cdots, a_{h}$ if and only if the largest eigenvalue of $M$ is $(2 h-1)^{1 / 2} h^{-1}$. In this case the spectrum of $M$ is the interval $\left[-(2 h-1)^{1 / 2} h^{-1},+(2 h-1)^{1 / 2} h^{-1}\right]$. (Received June 25, 1957.)

\section{Naoki Kimura: On some existence theorems in a multiplicative} system.

Let $A \in(\pi)$ denote the proposition that a multiplicative system $A$ satisfies the property $\pi$. Here we consider only properties which are satisfied by a multiplicative system consisting of only one element. Then the theorems are as follows: I. Let $\pi$ satisfy the following three conditions: (1) $A \in(\pi)$ implies every subsystem $\in(\pi)$, (2) If $A, B \in(\pi)$, then $A \times B \in(\pi)$ (or (2') If $A, B \in(\pi)$, then $A \times{ }^{c} B \in(\pi)$ for any nontrivial system $C$ ), (3) If $A_{\gamma} \in(\pi)$, then its inverse limit inv $\lim A_{\gamma} \in(\pi)$. Then for any given system there exists a greatest (or maximal) decomposition so that its quotient system satisfies the property $\pi$. II. Let $\pi$ satisfy the following three conditions: (1) $A \in(\pi)$ implies every quotient system $\in(\pi)$, (2) If $A, B \in(\pi)$, then $A * B \in(\pi)$ (or (2') If $A, B \in(\pi)$, then $A *_{C} B \in(\pi)$ for any system $C$ ), (3) If $A_{\gamma} \in(\pi)$, then its direct limit $\operatorname{dir} \lim A_{\gamma} \in(\pi)$. Then for any given system there exists a greatest (or a disjoint family of maximal) subsystem(s), (any of) which satisfies the property $\pi$. The notion of a direct product with amalgam, $A \times{ }^{c} B$, and a free product with amalgam, $A *{ }_{c} B$, are considered. A generalization of the above theorems are also stated. Further several applications will be given. (Received June 28, 1957.)

\section{9t. L. A. Kokoris: Some nodal noncommutative Jordan algebras.}

Let $A$ be a noncommutative Jordan algebra over a field $F$ with an identity $e$ and let $A=e F+N$ where every element of $N$ is nilpotent. If $N$ is not a subalgebra of $A, A$ is called a nodal algebra by $\mathrm{R}$. D. Schafer. Schafer has proved that nodal noncom- 
mutative Jordan algebras of characteristic zero do not exist (see Noncommutative Jordan algebras of characteristic 0, Proc. Amer. Math. Soc. vol. 6 (1955) pp. 472-475). In this paper simple nodal algebras are constructed for every characteristic different from zero. These examples have the property that the associated algebras $\mathrm{A}^{+}$are associative. (Received May 6, 1957.)

\section{0t. George Kolettis, Jr.: A generalization of Ulm's theorem.}

If $G$ is a reduced primary abelian group, then its Ulm invariants characterize the group in case $G$ is countable. (Ulm's theorem, cf. Kaplansky, Infinite Abelian groups). It is shown that the Ulm invariants are still sufficient to characterize the group in the uncountable case, provided $G$ can be expressed as a direct sum of countable groups. This is accomplished by a reduction to the case where $G$ has cardinality $\boldsymbol{N}_{1}$. Necessary and sufficient conditions are obtained for a set of cardinals to be the Ulm invariants of a direct sum of countable groups. (Received July 12,1957.)

\section{1t. R. C. Lyndon: On special Horn sentences.}

For terminology see two abstracts presented at the April 1957 meeting in Berkeley. A special Horn sentence is a sentence obtained by universal quantification and conjunction from formulas of the form $P \rightarrow a$ where $P$ is a positive formula and $a$ an atomic formula. Let $S$ and $T$ be two sentences, and form $S(z)$ from $S$ by replacing each part $\phi_{i}\left(t_{1}, \cdots, t_{r_{i}}\right)$ of $S$ by $\pi_{i}\left(z, t_{1}, \cdots, t_{r_{i}}\right)$, where $z$ is a new variable and the $\pi_{i}$ are distinct new predicates. Form $T^{\prime}$ from $T$ by replacing each part $\phi_{i}\left(t_{1}, \cdots, t_{r_{i}}\right)$ by $\forall z \cdot \pi_{i}\left(z, t_{1}, \cdots, t_{r_{i}}\right)$. Then $S$ strongly implies $T$ if $\forall z \cdot S(z)$ implies $T^{\prime}$. Theorem 1 . If $M$ is a special Horn sentence, then $M$ strongly implies $M$. Theorem 2. If $S$ strongly implies $T$, then there exists a special Horn sentence $M$ such that $S$ implies $M$ and $M$ implies $T$. The main tool of the proof is a detailed application of the Gentzen calculus. (Received July 10, 1957.)

\section{2t. R. C. Lyndon: On subdirect products of relational systems.}

For terminology see preceding abstract. A subsystem $Q^{\prime}$ of the direct product $Q$ of systems $Q_{i}$ is a subdirect product of the $Q_{i}$ if each natural homomorphism $Q_{\text {onto }}$ $Q_{i}$ maps $Q^{\prime}$ onto $Q_{i}$. For a class $K$ of systems, let $P K$ be the class of all systems isomorphic to a subdirect product of systems from $K$. Lemma. $Q=\langle A, R, \cdots\rangle$ is isomorphic to a subdirect product of systems $Q_{2}$ if and only if there exist systems $B$ and $B_{i}$ with congruences $C, C_{i}$ such that $Q \cong B / C$, each $Q_{i} \cong B_{i} / C_{i}$, where each $B_{i}=B$ and $\cap C_{i}=C, \cap R_{i}=R, \cdots$. (Operations add no complication.) Theorems. 1. If a special Horn sentence holds in $K$, then it holds in $P K$. 2. If, for all $K$ such that $S$ holds, $T$ holds in $P K$, then there exists a special Horn sentence $M$ such that $S$ implies $M$ and $M$ implies $T$. 3. If $K$ is an arithmetical class, then $A P K$ is characterized by those special Horn sentences that hold in $K$. 4. If $K$ is arithmetical, then $K=P K$ if and only if $K$ is characterized by special Horn sentences. 5 . There exists arithmetical $K$ such that $P K$ is not arithmetical. (Received July 10, 1957.)

\section{H. B. Mann: On integral bases.}

Let $J$ be a Dedekind domain, $F$ its quotient field. Let $F^{\prime}$ be a quadratic extension of $F$ and let $J^{\prime}$ be the domain of elements of $F$ which satisfy monic equations with coefficients in $J$. The domain $J^{\prime}$ will be called an algebraic extension of $J$. If $F$ has characteristic different from 2 then $J^{\prime}$ has an integral basis over $J$ if and only if $F^{\prime}=F\left(D^{1 / 2}\right)$ where $D$ is the discriminant of $F^{\prime}$ over $F$. If $F$ has characteristic 2 then 
the different of $F^{\prime}$ over $F$ is an ideal of $J$ and $J^{\prime}$ has an integral basis over $J$ if and only if this ideal is principal. If $J$ contains an ideal which is not principal then it is possible to construct a quadratic extension $J^{\prime}$ over $J$ which has no integral basis. Hence every algebraic extension of $J$ has an integral basis if and only if every ideal of $J$ is principal. (Received July 2, 1957.)

644t. M. D. Marcus, B. N. Moyls and R. Westwick: Some extreme value results for indefinite Hermitian matrices. II.

Let $H$ be an $n$-square Hermitian matrix with eigenvalues $\lambda_{1} \geqq \lambda_{2} \geqq \cdots \geqq \lambda_{n}$; and let $E_{r}\left[a_{1}, \cdots, a_{k}\right]$ be the $r$ th elementary symmetric function of the numbers $a_{1}, \cdots, a_{k}$. It is shown that the maximum and minimum values of $E_{r}\left[\left(H x_{1}, x_{1}\right), \cdots\right.$, $\left.\left(H x_{k}, x_{k}\right)\right]$, as $x_{1}, \cdots, x_{k}$ range over all sets of $k$ orthonormal vectors $(1 \leqq r \leqq k \leqq n)$, have the form $E_{r}\left[b_{1}, \cdots, b_{k_{1}}, \cdots, b_{k q}\right], 0=k_{0}<k_{1}<\cdots<k_{q}=k$, where $b_{k_{j}+1}$ $=\cdots=b_{k_{j+1}}=\left(\mu_{k_{j+1}}+\cdots+\mu_{k_{j+1}}\right) /\left(k_{j+1}-k_{j}\right)$ for $0 \leqq j \leqq q-1$, and $\mu_{1}, \cdots, \mu_{k}$ are the first $s$ and last $k-s$ of the $\lambda$ 's $(0 \leqq s \leqq k)$. (Received July 1, 1957.)

645t. Anthony Mardellis: The monodromic group and the PicardVessiot theory. Preliminary report.

The author's purpose is to show the place occupied by the monodromic group of a differential equation in the Picard-Vessiot theory as presented by E. R. Kolchin (Ann. of Math. (2) vol. 49 (1948) pp. 1-42). Consider the differential equation of order $n: \sum_{i=0}^{n} Q_{i}(z) d^{n-i} w(z) / d z^{n-i}=0, Q_{0}(z)=1$, with coefficients in the field of rational functions $\mathcal{F}$. Let $w^{1}, w^{2}, \cdots, w^{n}$ be a fundamental system of solutions. Furthermore, let $\mathcal{G}=\mathcal{F}\left\langle w^{1}, w^{2}, \cdots, w^{n}\right\rangle$, and let $g$ denote the group of all automorphisms of $\mathcal{G}$ over $\mathcal{F} ; \mathcal{F}_{1}$ the subfield of all one-valued functions contained in $\mathcal{G}$; $g\left(\mathfrak{F}_{1}\right)$ the subgroup of $g$ consisting of all automorphisms which leave invariant every element of $\mathcal{F}_{1}$. The monodromic group $M$ is a group of automorphisms of $\mathcal{G}$ over $\mathcal{F}_{1}$, and $g\left(F_{1}\right)$ is the smallest algebraic subgroup of $g$ containing $M . g=M \leftrightarrow$ all solutions $w^{1}, w^{2}, \cdots, w^{n}$ in a fundamental system are algebraic. If $\mathcal{F}=\mathcal{F}_{1}$, then $M$ can replace $g$ in the following two theorems: "The equation is reducible over $\mathcal{F} \leftrightarrow g$ is reducible"; "All integrals of the equation are algebraic $\leftrightarrow g$ is finite." The class of equations for which $\mathcal{F}=\mathscr{F}_{1}$ includes all equations which are everywhere of the Fuchs type. If two equations belong to this class, and have the same monodromic group $M=M^{\prime}$, then $g=g^{\prime}$. (Received July 3, 1957.)

646. E. D. Nering: An application of results on the reduction of an algebraic function field to extensions of the field of constants of an algebraic function field.

This paper develops the application of a previous paper, Reduction of an algebraic function field modulo a prime in the constant field (unpublished), to the extensions of the field of constants of an algebraic function field. In the earlier paper a formula of the form $g-1=\sum_{i=1}^{s} r_{i} e\left(H_{i}\right)\left(g_{i}-1\right)+\rho$ was obtained in which $g$ is the genus of a given algebraic function field $K$ and the $g_{i}$ are the genera of fields obtained by reducing $K$ modulo extensions of a prime in the constant field. In the present paper an algebraic extension of the constant field is obtained by first extending the field by adjoining a transcendental element $\Lambda$, and then reducing this field modulo a prime corresponding to the irreducible polynomial $h(\Lambda)$ for the algebraic element $\lambda$. For such a prime $s=1$ and $e\left(H_{1}\right)=1$. If either $\lambda$ or $K$ is separable over the constant field, then $r_{1}=1$. If $\lambda$ is separable, $\rho=0.2 \rho$ is the degree of the divisor of a conductor. Tate 
(Proc. Amer. Math. Soc. vol. 3 (1952) pp. 400-406) has discussed the inseparable case, and in his results the term corresponding to the $2 \rho$ is the degree of the divisor of a different. The two methods are entirely analogous; the conductor is determined in a way which is, in a sense, "dual" to the way in which the different is determined. The conductor is defined in an invariant manner. (Received July 10,1957.)

\section{7t. Anil Nerode: Composita, equations, and freely generated alge- bras.}

Define a $V$-compositum $T$ to consist of a set $T$, a nonempty subset $V$ of $T$, and a set $S$ of maps on $T$ to $T$ such that: $S$ is closed under composition and contains the identity; every map on $V$ to $T$ has exactly one extension in $S$. Relative to suitable definitions every compositum has a regular representation as a subcompositum of a concrete compositum. In logic this theory yields a generalization to composita of the semantic completeness of the calculus of identities of Birkhoff. In algebra this theory yields: rigorous existence and uniqueness proofs for freely generated abstract algebras implying the equivalence of characterizations either in terms of endomorphism structure or mapping properties or true identities; and a subdirect sum representation for freely generated algebras a nalogous to Birkhoff's for general abstract algebras. Further, these imply what appears to be a solution to problem 68 of Birkhoff. If $G$ has $\alpha$ elements, the free distributive lattice on $\alpha$ generators is the sublattice of $P(P(G)$ ) generated by $W_{\sigma}$ for $g$ in $G$, where $W_{\theta}$ consists of all subsets of $G$ containing $g$. (Received July 8,1957 .)

\section{8t. H. A. Osborn: Lie algebras of alternating forms.}

Let $E$ be a finite-dimensional vector space over a field of characteristic zero, $E^{\prime}$ the dual of $E$, and $S$ a nondegenerate symmetric bilinear form on $E^{\prime} \times E^{\prime}$. If $A$ and $B$ are any two alternating bilinear forms on $E \times E$ then one can define a new alternating bilinear form $[A, B]_{S}$ on $E \times E$ such that, in terms of an arbitrary basis, $\left([A, B]_{S}\right)$ $=(A)(S)(B)-(B)(S)(A)$, where parentheses indicate the appropriate matrix representations. Using the correspondence $(A) \leftrightarrow(S)(A)$, which can also be defined independently of the basis, any such Lie algebra of alternating forms is isomorphic to a Lie algebra of endomorphisms of $E$. There exist many Lie algebras of endomorphisms of $E$ which cannot be obtained in this way, however, so that the following result is a considerably sharpened form of Ado's theorem. THEOREM: Any Lie algebra over a field of characteristic zero possesses a faithful representation by an algebra of alternating bilinear forms. COROLLARY: Any Lie algebra over an algebraically closed field of characteristic zero possesses a faithful matrix representation (in the usual sense) by a Lie algebra of skew-symmetric matrices. (Received June 5, 1957.)

\section{Martin Pearl: On normal matrices.}

Let $F$ be an arbitrary field and let $a \leftrightarrow \bar{a}$ be an involutory automorphism of $F$. The conjugate transpose $A^{*}=\left[b_{i j}\right]$ of the matrix $A=\left[a_{i j}\right]$ is defined by $b_{i j}=\bar{a}_{i i} . A$ is said to be normal if $A A^{*}=A^{*} A$ and is said to be an $E P r$ matrix if $A$ has rank $r$ and if $A \xi=0$ implies $A^{*} \xi=0$ (H. Schwerdtfeger, Introduction to linear algebra and the theory of matrices). By means of $E P r$ matrices, some of the classical results concerning real and complex normal matrices are generalized to $F$. It is shown that a necessary and sufficient condition that a matrix $A$ which has the same rank as $A A^{*}$ be normal is that there exist a unitary matrix (i.e. $U U^{*}=U^{*} U=I$ ) $U$ such that $A^{*}=U A=A U$. Let $K$ be a field containing $F$ and all of the characteristic roots of $A$. Furthermore, let $A-\lambda I$ and $(A-\lambda I)(A-\lambda I)^{*}$ have the same rank for all $\lambda \in K$ and let $A-\lambda I$ be an 
$E P n_{\lambda}$ matrix, where $r_{\lambda}$ is the rank of $A-\lambda I$, for all $\lambda \in K$. Then $A$ is normal and there is a polynomial $f$ such that $A^{*}=f(A)$. (Received July 11, 1957.)

650. E. C. Posner: Differentiably simple rings.

Let $R$ be a commutative ring and $D$ a family of derivations of $R$ such that $R$ has no ideals invariant under every derivation in $D$. Then $R$ is an algebra with unit such that every zero divisor is nilpotent; if $R$ is of characteristic zero, then $R$ has no divisions of zero. The ring of differential polynomials in $D$ over $R$ made faithful on $R$ is simple; if the elements of $D$ commute and are linearly independent over $R$, the ring of commutative differential polynomials is this faithful ring. (Received July 10 , 1957.)

\section{G. B. Preston: Representations of semigroups.}

In a recent paper M. P. Schützenberger (C. R. Acad. Sci. (Paris) vol. 244 (1957) pp. 1994-6) showed that every elementary (loc. cit.) D-class $D$ in a semigroup with identity $S$ determines a pair of left-right dual semigroups of matrices $M(D)$ and $N(D)$, say, and a pair of homomorphisms $f_{D}: S \rightarrow M(D)$ and $g_{D}: S \rightarrow N(D)$ of $S$ onto these semigroups. We show that the restriction to elementary D-classes is unnecessary. Let $D_{0}$ be the set of all $D$-classes in $S$ and denote by $M(N)$ the direct product of all the $M(D)(N(D))$ for $D \in D_{0}$. Then $M$ and $N$ can also be regarded as semigroups of matrices. Define $f: S \rightarrow M$ by $(s f)_{D}=s f_{D}$, for $s \in S$, where for $m \in M, m_{D}$ denotes the component of $m$ in $M(D)$. Similarly define $g: S \rightarrow N$ by $(s g)_{D}=s g_{D}$, and $f \times g: S$ $\rightarrow M \times N$ by $s(f \times g)=(s f, s g)$. We find necessary and sufficient conditions for $f, g$ and $f \times g$ to be faithful, i.e. to be one-to-one. For regular semigroups $f \times g$ is faithful. For inverse semigroups $f$ and $g$ are each faithful. (Received July 11,1957.)

\section{2t. J. B. Roberts: A new proof of a theorem of Lehmer.}

The following theorem is proved. Let $\alpha_{0}, \cdots, \alpha_{k}$ be an arbitrary set of $k+1$ complex numbers (distinct or not) and let $b$ be an integer $\geqq 2$. Let $C$ be the collection of all numbers of the form $j_{0} \alpha_{0}+\cdots+i_{k} \alpha_{k}$ where the $j_{i}$ are integers satisfying $0 \leqq j_{i} \leqq b-1$. Further let $C_{j}, 0 \leqq j \leqq b-1$, be the collection of elements of $C$ for which $j_{0}+\cdots+j_{k} \equiv j(\bmod b)$. Then if $P(x)$ is a polynomial of degree smaller than or equal to $k, \sum_{n \in C i} P(x+n)$ is equal to a constant independent of $i, 0 \leqq i \leqq b-1$. This theorem is equivalent to a theory of Lehmer (Scripta Mathematica vol. 13 (1947) pp. 37-41). The proof given here, while in some respects similar to that of Lehmer, makes use of difference operators while his proof makes use of differentiation. We also give a quick method for the calculation of the classes $C_{j}$ in the special case where $\alpha_{i}=b^{i}$. (Received June 24, 1957.)

\section{3t. J. B. Roberts: Some classes of orthogonal functions.}

The author has proved two identities of the form $\sum_{n=0}^{k+1, b-1} c_{b}(n) P(x+n)=0$ for all polynomials of sufficiently small degree. One is proved in the paper discussed in the preceding abstract and the other in the Amer. Math. Monthly vol. 64 (1957) pp. 317-322. We shall call these identities I and II respectively. For each $n \geqq 0$ define the function $w_{n}(x)$ to be of period 1 , constant over intervals of length $1 / b^{n+1}$, and taking the value of the $i$ th coefficient in I on the $i$ th interval of length $1 / b^{n+1}$ in $[0,1)$. Define $t_{n}(x), n \geqq 0$, in the same way using the coefficients in II. Then it is shown that $w_{n}(x)=\prod_{j=0}^{n} r_{j}(x)$ where $r_{j}(x)$ is the $j+1$ st Rademacher function of order $b$. Hence $\left\{w_{n}(x)\right\}$ is a lacunary subsequence of the Walsh functions of order $b$ and constitutes an orthonormal set. Further there is a class of functions $s_{j}(x), j \geqq 0$, such that: (a) if 
$Q(x)$ is a power product of the $s_{j}(x)$ with at least one exponent 1 then $\int_{0}^{1} Q(x) d x=0$; $t_{n}(x)=\prod_{i=0}^{n} s_{j}(x)$. Hence $\left\{t_{n}(x)\right\}$ is an orthogonal set. However while the Walsh functions of order $b$ are the completion of the Rademacher functions of order $b$ one cannot complete the set of $s_{j}(x)$ in the same way. Their completion would be of interest. (Received June 24, 1957.)

\section{4t. Alex Rosenberg and Daniel Zelinsky: Tensor products of semi- primary algebras.}

Theorem: Let $A$ and $B$ be algebras over a field $F$. If $A \otimes B$ is semiprimary then either $A$ is semiprimary or $B$ is radical. Here "semiprimary" means that $A$ modulo a radical ideal is semisimple with minimum condition; "radical" may be taken to mean either quasi-regular, nil or nilpotent. Under the same hypotheses, if $B$ is the opposite of $A$ and "radical" means "nilpotent," then $A$ modulo its maximal nilpotent ideal is finite over $F$. (Received May 20,1957.)

\section{N. J. Rothman: Characters of mobs. Preliminary report.}

If $S$ is an abelian mob (=topological semigroup), $S^{*}$, the set of all bounded continuous homomorphisms to the multiplicative complex plane, is called the characters of $S . S^{*}$ with the compact open topology and multiplication defined by $f g(x)$ $=f(x) g(x)$ is a mob with uniformly continuous multiplication. For $A \subset S,\left(S^{*}: A\right)$ $=\left[f \in S^{*}: f \mid A=0\right]$ is a closed ideal in $S^{*}$. If $I$ is an ideal in $S$ then $\left(S^{*}: I\right)$ is topologically isomorphic to $(S / I)^{*} \backslash\{1\}\left(S / I\right.$ is the Rees quotient); also $I^{*}$ is algebraically isomorphic to $S^{*} /\left(S^{*}: I\right)$. If $\left\{S_{i}\right\}_{i=1}^{n}$ is a finite collection of mobs with the identities $e_{i}$ then the character mob of their product is topologically isomorphic to a Rees quotient of the product of the character mobs $S_{i}^{*}$. An abelian mob $S$ with 0 possesses nontrivial characters if and only if $S$ contains an ideal $I$ and there is a closed $R S T$ submob $R$ of $I \times I$ with $I / R$ isomorphic to a nondegenerate submob of the usual unit interval (Cf. Wallace: Tulane, Notes on mobs). (Received July 11, 1957.)

\section{6t. R. D. Schafer: On noncommutative Jordan algebras.}

A power-associative algebra $A$ with 1 over a field $F$ is called a nodal algebra in case every element of $A$ is of the form $\alpha 1+z$ where $\alpha \in F$ and $z$ is nilpotent, and $A$ is not of the form $A=F 1+N$ for $N$ a nil subalgebra of $A$. Let $K$ be the algebraic closure of $F$. If $A$ is a noncommutative Jordan algebra (Proc. Amer. Math. Soc. vol. 6 (1955) pp. 472-475) over $F$ of characteristic $\neq 2$ such that $A_{K}$ is without nodal subalgebras, then $A$ is trace-admissible. A structure theory for such algebras is obtained. The paper concludes with some theorems about nodal noncommutative Jordan algebras (which are necessarily of characteristic $p$ ). That nodal noncommutative Jordan algebras exist has recently been shown by L. A. Kokoris (see abstract 639t). (Received May 27, 1957.)

\section{R. D. Schafer: Restricted noncommutative Jordan algebras of characteristic $p$.}

Commutative Jordan algebras of characteristic $p>2$ satisfy the identity $R_{x}^{p}=R_{x} p$ where $R_{x}$ denotes a right multiplication. Noncommutative Jordan algebras of characteristic $p$ do not in general satisfy this identity. Those which do are called restricted noncommutative Jordan algebras of characteristic $p$ (by analogy with the terminology for Lie algebras). It is proved that this class does not contain nodal algebras, so the results of the preceding abstract may be applied to obtain a structure theory. (Received May 27, 1957.) 


\section{8t. A. Sharma: q-Bernoulli and Euler numbers of higher order.}

The object of this note is to extend the recent results of L. Carlitz [Duke Math. J. vol. 15 (1948) pp. 987-1000 and Trans. Amer. Math. Soc. vol. 76 (1954), pp. 332350 ] on $q$-Bernoulli and Eulerian numbers to such numbers of higher order. For want of a natural generating function for the numbers introduced by Carlitz such an extension is not immediately obvious. We define numbers $\beta_{m}^{(\alpha, k)}$ for $h, k$ integers $\geqq 1$, $m \geqq 0$ such that $\beta_{m}^{(h, k)}=\beta_{m}^{(h-1, k)}+(q-1) \beta_{m+1}^{(h-1, k)}$ and $\beta_{m}^{(0, k+1)}=(m-k) /[m-k] \beta_{m}^{(0, k)}$ where $[x]=\left(q^{x}-1\right) /(q-1)$. The numbers $\beta_{m}^{\left(h_{k}\right)}$ and the corresponding polynomials in $q^{x}$ reduce for $q=1$ and $h=k$ to the $\beta_{m}^{(k)}$ - the Bernoulli numbers, etc. of higher order. Euler numbers and polynomials of higher order and their interrelations are also studied. A sort of von Staudt Clausen theorem also holds when $h>k=1$. (Received July 8,1957 .)

\section{9t. Abraham Spitzbart: A generalization of Hermite's interpola- tion formula.}

Hermite's interpolation formula (cf. Z. Kopal, Numerical analysis, New York, 1955) provides an explicit representation of a polynomial of minimum degree which assumes given functional and first derivative values at specified points. The present paper generalizes this result to include the values of derivatives up to specified, arbitrary orders at the given points. The desired polynomial can be written in the form $\sum_{j=0}^{n} \sum_{k=0}^{r_{j}} A_{j k}(x) f_{j}^{(k)}$ where $x_{j}, r_{j}, f_{j}^{(k)}, j=0,1, \cdots, n ; k=0,1, \cdots, r_{j}$ are given. If $P_{j}(x) \equiv \prod_{i=0}^{\prime n}\left(x-x_{i}\right)^{r_{i}+1}$, where the prime indicates the omission of $\left(x-x_{j}\right)^{r_{j}+1}$, and $g_{j}(x) \equiv[p(x)]^{-1}$, the result is $A_{j k}(x)=p_{j}(x)\left(x-x_{j}\right)^{k}(k !)^{-1} \sum_{i=0}^{r_{i}^{-k}}(t !)^{-1} g_{j}^{(t)}\left(x_{j}\right)\left(x-x_{j}\right)^{t}$. This result then enables one to give an explicit formula for the general divided difference with repeated arguments, of any order. (Received July 5, 1957.)

\section{Seth Warner: Ulam's Axiom and Cartesian products of fields.}

Ulam's Axiom is the assertion that there exists no (countably additive) nonzero measure $m$ defined on the set of all subsets of some set, taking on only the values 0 and 1 , such that $m(F)=0$ for all finite subsets $F$. If $K$ is a field and $A$ a set, let $K(A)$ be the Cartesian product of $\left(K_{\alpha}\right), \alpha \in A$, where $K_{\alpha}=K$ for all $\alpha$. We call a homomorphism from the $K$-algebra $K(A)$ into $K$ a multiplicative linear form on $K(A)$. 1. If the cardinality of $A$ does not exceed that of $K$, every nonzero multiplicative linear form on $K(A)$ is a projection. 2. Ulam's Axiom implies that if $K$ is an infinite field, every nonzero multiplicative linear form on $K(A)$ is a projection. 3. Let $K$ be a countably infinite field. Then Ulam's Axiom is equivalent to the assertion that for any set $A$, every nonzero multiplicative linear form on $K(A)$ is a projection. 4. If $K$ is a finite field, there exists a canonical one-to-one correspondence between the nonzero multiplicative linear forms on $K(A)$ and the ultrafilters on $A$; in particular, if $A$ is infinite, there exist nonzero multiplicative linear forms on $K(A)$ which are not projections. (Received July 5, 1957.)

\section{1t. F. B. Wright: Some remarks on Boolean duality.}

The duality of homomorphisms between Boolean algebras with continuous functions and Boolean spaces was established by M. H. Stone (Trans. Amer. Math. Soc. vol. 40 (1936) pp. 37-111). This has been extended recently to a duality of hemimorphisms with Boolean relations, by P. R. Halmos (Comp. Math. vol. 12 (1955) pp. 217-249). The purpose of this note is to show that Halmos' extension has essentially reached the limits of this sort of duality. Let $f$ be any mapping of one 
Boolean algebra into another, and let $\phi$ be any relation from one Boolean space to another. Following Halmos, the duals $f^{*}$ and $\phi^{*}$ can be defined; $f^{*}$ is a relation and $\phi^{*}$ is a mapping. (1) A necessary and sufficient condition that $f=f^{* *}$ is that $f$ be a hemimorphism. (2) A necessary and sufficient condition that $\phi=\phi^{* *}$ is that $\phi$ be a Boolean relation. In both cases, the sufficiency of these conditions is due to Halmos, and it requires only slightly more delicate considerations to establish the necessity of these conditions. (For example, the ranges of $f^{*}$ and $\phi^{*}$ must be handled with care.) This analysis serves to extend the Jonsson-Tarski duality theorem (Amer. J. Math. vol. 73 (1951) pp. 217-249), and yields an alternative proof of the McKinsey-Tarski representation of closure algebras (Ann. of Math. vol. 45 (1944) pp. 891-939). (Received July 8,1957 .)

\section{AnAlysis}

\section{2t. H. A. Antosiewicz: Reducible linear differential systems, I.}

The main result proved is the following theorem for complex $n$-vector equations $\dot{x}=[C+B(t)] x$ where $C=\operatorname{diag}\left(\lambda_{1}, \cdots, \lambda_{n}\right)$ and $B(t)=\left(b_{i j}(t)\right), 1 \leqq i, j \leqq n$, is defined and continuous on the (real) half-line $L=(0, \infty)$ : If for some index $q, 1 \leqq q \leqq n$, (1) $\operatorname{Re}\left(\lambda_{i}-\lambda_{q}\right) \neq 0$ for all $i \neq q$, (2) sup $\left|\int^{t} b_{q q}(s) d s\right|<\infty$ on $L$, and (3) $\int^{\infty}\left|b_{i j}(s)\right|{ }^{\gamma} d s<\infty$ for some $\gamma, 1 \leqq \gamma \leqq 2$, and all $(i, j) \neq(q, q)$, there is a solution $x_{q}(t)=\left(x_{1 q}(t), \cdots, x_{n q}(t)\right)$ which, as $t \rightarrow \infty$, satisfies $x_{i q}(t) \exp \left(-\lambda_{q} t-\delta_{i q} \int_{0}^{t} b_{q q}(s) d s\right) \rightarrow \delta_{i q}, 1 \leqq i \leqq n$. This solution is unique provided the set $P_{g}$ of indices $p$ such that $\operatorname{Re}\left(\lambda_{q}-\lambda_{p}\right)>0$ is empty. If $P_{q} \neq 0, x_{q}(t)$ may be determined so as to satisfy, at any $t_{0}>0$ sufficiently large, $x_{p q}\left(t_{0}\right)=c_{p}, p \in P_{q}$, with arbitrary constants $c_{p}$. It follows that if the hypotheses hold for all $q, 1 \leqq q \leqq n$, the equation $\dot{x}=[C+B(t)] x$ is reducible in Lyapunov's sense [Annals of Mathematics Studies, no. 17, Princeton University Press, 1947, p. 242], i.e., a base of solutions, $X(t)$, has the form $X(t)=Z(t) e^{C t}$ where $Z(t)$ is, on $L$, nonsingular, continuously differentiable and bounded together with $Z^{-1}(t)$. This generalizes a theorem due to Erugin [Trudy Mat. Inst. V. A. Steklov vol. 13, 1946, $93 \mathrm{pp}$.$] and complements results of Yakubovic [Dok. Akad. Nauk SSSR vol. 66$ (1949) pp. 577-580] and of Wintner [Amer. J. Math. vol. 77 (1955) pp. 45-86]. (Received July $3,1957$.

\section{3t. W. D. L. Appling: Concerning integrals. Preliminary report.}

Suppose each of $f$ and $g$ is a real function on $[a, b]$, each of $k$ and $J$ a number and, if $h>k$, there is a chain, $C$ from $a$ to $b$ such that, for each refinement $\left(x_{i}\right)_{i=0}^{n}$ of $C$, $\left|J-\sum_{i=1}^{n}(1 / 2)\left(f\left(x_{i}\right)+f\left(x_{i-1}\right)\right)\left(g\left(x_{i}\right)-g\left(x_{i-1}\right)\right)\right|<h$. There is a least such $k$ and, for this $k$, only one $J$, denoted by $\int_{a}^{b} f d g\left(=\left.f g\right|_{a} ^{b}-\int_{a}^{b} g d f\right)$. Properties of this integral are developed. Classes of functions $f$ are characterized by means of the integral, e.g., (1) every bounded $f$ is integrable with respect to any $g$ of bounded variation, and if every continuous $f$ is $g$-integrable, then $g$ is of bounded variation and (2) $g$ is constant on no subinterval and bounded if and only if every $g$-integrable $f$ is bounded. (Received June 24, 1957.)

664. R. W. Bass: A global structure theorem for arbitrary dynamical systems in $E^{n}$.

Notations: Bull. Amer. Math. Soc. Abstracts 63-3-358 through 63-3-361 and 63-3-385, 63-3-386. Def. The phase-space $G$ of an elementary system is the product of $E^{1}$ with a connected region of an (n-1)-dimensional manifold; $E^{1}$ acts on $G$ as a translation group. The flow in $G$ is P-irrotational, wandering (G. D. Birkhoff), has 
no improper saddle-point (Nemickii), hence is dispersive (Montgomery-Zippin). Defs. (Birkhoff) The center $C$ of $S$ is the largest closed set possessing recurrence of relative domains, i.e. the closure of all Poisson-stable orbits. The hull $H \equiv S-C$. Def. An (asymptotic) orbit of the hull is a separatrix if not "orbitally asymptotically stable." Def. $W$ (for walls) is the (closed) set of all separatrices. For compact conservative $S$ 's, $H=\phi$; but analysis of $H$ for dissipative $S$ 's is important. Th. $S=C \cup H, C \cap H=\phi$; $H=W \cup G, W \cap G=\phi ; G=\bigcup_{\alpha} G_{\alpha}, G_{i} \cap G_{j}=\delta_{i j}$, where each $G_{\alpha}$ is an elementary system. Cor. (a) NASC's for global asymptotic stability of equilibrium $\left(C=x_{0}\right)$; (b) inversion of Lyapunov's Direct Theorems; (c) planar decomposition theory of Poincaré, Bendixson, and Markus (Trans. Amer. Math. Soc. vol. 76 (1954) pp. 127148.) (Received July 12, 1957.)

\section{Errett Bishop: A generalization of a theorem of Mergelyan. Preliminary report.}

Let $C$ be a compact set of the complex plane, whose complement is connected. We investigate the structure of a Borel measure $\mu$, on the boundary of $C$, having the property that $\int f(z) d \mu(z)=0$ for all polynomials $f$. The measure $\mu$ can be written as an infinite sum, $\mu=\sum_{i=1}^{\infty} \mu_{i}$, with convergence in the norm topology for measures, where each of the Borel measures $\mu_{i}$ has the following structure. There exists a component $U_{i}$ of the interior of $C$, such that $\mu_{i}$ is a measure on the boundary of $U_{i}$ and such that the analytic function $\phi_{i}$ on $U_{i}$ defined by $\phi_{i}(z)=(1 / 2 \pi i) \int d \mu_{i}(\zeta) /(\zeta-z)$ has the following property: there exists a sequence $\left\{\gamma_{j}\right\}$ of simple closed rectifiable curves lying in $U_{i}$ such that $\int_{\gamma_{j}} g(z) \phi_{i}(z) d z \rightarrow \int g(z) d \mu_{i}(z)$ as $j \rightarrow \infty$, for each continuous function $g$ on $C$. We say that $\mu_{i}$ is the boundary measure of the differential $\phi_{\imath}(z) d z$. As a corollary of this theorem, one obtains the theorem of Mergelyan, that any continuous function on $C$ which is analytic at interior points can be uniformly approximated by polynomials. (Received July 22, 1957.)

\section{R. C. Buck: Converse forms of the Hadamard product theorem.}

If $F(z)$ and $G(z)$ are regular everywhere, except for isolated singularities at $\alpha$ and $\beta$, respectively, then their Hadamard product $H(z)$ is regular everywhere except at $\alpha \beta$. The following partial converse is obtained: Let $H(z)$ be regular everywhere except at $\gamma$, and be the Hadamard product of functions $F(z)$ and $G(z)$; if $F$ and $G$ are required to have their singularities confined to some bounded set in the right half plane, and $\gamma$ is either a pole or an essential singularity of finite exponential order, then $F$ and $G$ must be of the same sort. This does not hold if $\gamma$ is unrestricted. (June 24, 1957.)

\section{7t. R. C. Buck: Derivations in operator algebras.}

Let $E$ be a locally convex linear space, and $\mathfrak{R}(E)$ be the algebra of continuous linear transformations, $E \rightarrow E$. A derivation of $\mathfrak{Q}(E)$ is a linear mapping $\partial$ of $\mathfrak{R}(E)$ into itself which obeys $\partial(A B)=\partial(A) B+A \partial(B)$. Any transformation $D \in R(E)$ gives rise to an inner derivation of $\mathcal{R}(E)$ defined by $\partial(A)=[D, A]=D A-A D$. Theorem 1 . The inner derivation $\partial$ obtained from $D$ is continuous in the bounded-open topology on $\mathfrak{R}(E)$. Theorem 2. Every continuous derivation of $\mathfrak{R}(E)$ is inner. The result extends also to transitive subalgebras of $\mathfrak{R}(E)$ which contain minimal left ideals. The proof depends upon an explicit construction of $D$ from $\partial$ which has been obtained jointly by the author and C. W. Curtis for the algebraic case, without topology. (Received June $24,1957$. 


\section{8t. R. C. Buck: Automorphisms of operator algebras.}

With notation as in the preceding abstract, it is shown that every automorphism $h$ of $\mathfrak{l}(E)$ which is continuous in the bounded-open topology has the form $h(T)$ $=D^{-1} T D$ for some nonsingular continuous $D \in \mathbb{R}(E)$. If $\mathfrak{A}$ is a transitive subalgebra of $\mathfrak{R}(E)$ with a minimal left ideal, then every continuous automorphism of $\mathfrak{A}$ can be extended to a continuous (inner) automorphism of $\mathfrak{R}(E)$. (Received June 24,1957 .)

\section{9t. R. C. Buck: A Stone-Weierstrass theorem for $C^{*}[X]$.}

Let $X$ be a space that is locally compact, but not compact, and let $C^{*}[X]$ be the set of all bounded continuous real valued functions on $X$. Introduce into $C^{*}[X]$ the strict topology $\beta$. (See Proc. Amer. Math. Soc. vol. 3 (1952) pp. 681-687 and Bull. Amer. Math. Soc. Abstract 62-3-310.) It is now known that $\beta$ is locally convex, nonmetrizable, and topologically complete. Theorem: If $X$ is $\sigma$-compact, then any subalgebra of $C^{*}[X]$ which separates points of $X$, is strictly dense. When $X$ is not required to be $\sigma$-compact, the same is true for subalgebras of $C^{*}[X]$ whose closures (strict) contain a nonvanishing function. This condition is not necessary, for the subalgebra $C_{\infty}[X]$ of functions with compact carrier is strictly dense in $C^{*}[X]$. (Received July $3,1957$. )

670t. C. C. Camp: An integral equation whose kernel involves finite discontinuities along both diagonals.

The former theory of the equation $u(x)=\lambda \int_{0}^{1} K(x, s) u(s) d s$ with a discontinuity of 1 in $\partial K / \partial x$ on the line $s=x$ and of -1 along the line $s=-x$ has been extended so as to remove the restriction requiring symmetry to the line $x=1 / 2$. The present paper considers $u(x)=\lambda \int_{-1}^{1} K(x, s) u(s) d s$ with a unit jump along the main diagonal $s=x$ but a discontinuity of $-h(-x)<0$ along the other diagonal $s=-x$. With the usual restrictions of continuity of $h(x), K(x, s)$ and its derivatives, if $\left[h(-x) K_{x x}(-x, s)\right.$ $\left.-K_{x x}(x, s)\right] / p(x)$ where $p(x)=h(x) h(-x)-1 \neq 0$ possesses a Fredholm reciprocal kernel the problem may be reduced to an integro-differential functional system and then to a regular integro-differential system. The Green's function for the latter may be used to establish an expansion for an arbitrary function $f(x)$ with piecewise continuous derivative. If $p(x)=0$ the problem can still be solved in a variety of cases. (Received July 15, 1957.)

\section{1t. J. W. Carr, III : Generalized functional round-off error analysis.}

Generalized error bounds for (a) implicit iteration or functional solutions and (b) explicit iterations are obtained for the round-off error generated during the course of a computation. (a) An extension is made of the work of Weissinger and Collatz in applying classical functional analysis to determine truncation error bounds for functional iteration, to include round-off error at each stage. This is done for a general system of nonlinear functions of an arbitrary number of variables, using arbitrary norms; conditions are found to guarantee stability of the solution under round-off, and upper limits for the total error obtained. (b) For explicit iteration, a similar round-off error analysis provides error bounds for a general system of nonlinear equations, for arbitrary norms. In both cases if the norm of the iteration operator is bounded less than one, the total round-off error is bounded independent of the number of iterations. Particular applications of the theory include the Jacobi diagonalization procedure, previously analyzed in an unpublished work of Goldstine, the 
Gauss Elimination solution methods, and various techniques of numerical integration of ordinary and partial differential equations. (Received June 18, 1957.)

672. Lamberto Cesari and W. R. Fuller (p): Existence theorems for periodic solutions of weakly nonlinear Lipschitzian autonomous differential systems.

Consider the system $\left({ }^{*}\right) \ddot{x}_{j}+\sigma_{j}^{2} x_{j}=\epsilon q_{j}(x, \dot{x}, \epsilon), j=1, \cdots, n$, where $x=\left(x_{1}, \cdots, x_{n}\right)$, $('=d / d t), \sigma_{j}>0, m \sigma_{j}+\sigma_{k} \neq 0, j \neq k, j, k=1, \cdots, n ; m=0, \pm 1, \cdots$, and each $q_{j}(x, \dot{x}, \epsilon)$ defined in $Q=\left[\left|x_{j}\right| \leqq R,\left|\dot{x}_{j}\right| \leqq R, 0 \leqq \epsilon \leqq \epsilon_{0}\right]$ is bounded in $Q$, is Lipschitzian with respect to $x, \dot{x}$ and continuous with respect to $\epsilon$ in $Q$, and $q_{j}(-x, \dot{x}, \epsilon)=-q_{j}(x, \dot{x}, \epsilon)$. Then for every complex number $a, 0<|a|<R$, and for all $\epsilon, 0 \leqq \epsilon \leqq \epsilon_{1}\left(0<\epsilon_{1} \leqq \epsilon_{0}\right)$ and every $l=1, \cdots, n$, system $\left(^{*}\right)$ has a solution of the form $x_{l}=|a| \sigma_{l}{ }^{-1} \sin (\tau t+\phi)$ $+\epsilon W_{l}(\tau t+\phi, \epsilon), x_{k}=\epsilon W_{k}(\tau t+\phi, \epsilon), k \neq l, k=1, \cdots, n, \phi$ arbitrary, where all $W_{0}$ are continuous functions of $\tau t+\phi, \epsilon$, periodic of period $2 \pi$ in $\tau t+\phi$, where $\tau=\tau_{j}(a, \epsilon)$ is continuous in $a, \epsilon$, and $\tau_{j}(a, 0)=\sigma_{j}$. This statement and others give existence theorems for periodic solutions under the sole requirements of a Lipschitz condition and conditions of symmetry. These statements have been obtained by a new proof of the convergence of the casting out method of successive approximations first used by L. Cesari [Atti Accad. Italia (6) vol. 11 (1940) pp. 633-692], and then developed by L. Cesari, J. K. Hale, R. A. Gambill, H. R. Bailey [see, e.g., Bull. Amer. Math. Soc. Abstracts 60-1-118, 60-1-119, 60-4-487, 62-6-668, 62-6-669] for analytic linear and nonlinear, autonomous and periodic, differential systems, and used by W. R. Fuller for differential-difference systems [Bull. Amer. Math. Soc. Abstract 63-4-520]. (Received July 10, 1957.)

\section{V. F. Cowling: On series of Legendre and Laguerre Polynomials.}

In this paper we are concerned with the problem of determining a region of regularity for functions defined by series of Laguerre and Legendre polynomials. Such regions are determined in terms of the rate of growth, in certain directions, of analytic functions which generate the coefficients at the positive integers. The complicated nature of the results prohibits their presentation here. (Received July 15, 1957.)

\section{S. P. Diliberto: A new proof of C. L. Siegel's center theorem.}

In his Vorlesungen Uber Himmelsmechanik (Springer-Gottingen 1956) C. L. Siegel states and proves this remarkable theorem: Let $d x / d t=f(x) \cdots\left(^{*}\right)$ be analytic in $x=\left(x_{1}, \cdots, x_{2 n}\right)$ about $x=0, f(0)=0$, and Hamiltonian. If $f(x)=A x+$ higher order terms, let $\lambda_{1}, \cdots, \lambda_{n}$ be the characteristic roots of $A$. If the $\lambda_{i}$ are pairwise different, $\lambda_{1}$ purely imaginary, and $\lambda_{i} / \lambda_{1} \neq$ integer $i=2, \cdots, n$ then the system $\left({ }^{*}\right)$ has a one parameter family (analytic) of periodic solutions centering $x=0$. The author gives a simpler proof and drops the requirement that $\lambda_{i}$ be pairwise distinct. The possibility of a zero root (i.e. $\lambda_{i}=0$ for some $i>1$ ) is included with additional hypothesis. (Received July 15, 1957.)

\section{5t. W. F. Eberlein: An integral over function space.}

Real functions $x(t)=\sum_{0}^{\infty} x_{n} t^{n}(-1 \leqq t \leqq 1)$ with $\|x\|_{1}=\sum_{0}^{\infty}\left|x_{n}\right|<\infty$ may be identified with elements $x=\left(x_{0}, x_{1}, x_{2}, \cdots\right)$ of the sequence space $l_{1}$. Since the unit sphere $S$ of $l_{1}$ is compact under the weak ${ }^{*}$ topology $=$ topology of coordinatewise convergence, a countably additive measure on $S$ is induced by a positive linear functional $E$ (integral) on $C(S)$, the weak* continuous functions on $S$. There exists a "natural" 
integral over $S$ reducing to $E(f)=2^{-1} \int_{-1}^{1} f\left(x_{0}\right) d x_{0}$ when $f$ is a function of $x_{0}$ alone. This integral has numerous applications to harmonic analysis and the theory of numerical integration, in particular. (Received July 11, 1957.)

676. Albert Edrei (p) and W. H. J. Fuchs: On the lower order of meromorphic functions with deficient values.

Let $f(z)$ be meromorphic and $\sigma>1$. Using the standard notations of the theory of R. Nevanlinna: $T(r) \leqq\{4 /(\sigma-1)\} T(\sigma r)+\max \left\{N(\sigma r, 0)+N\left(\sigma r_{1} \infty\right)\right\}+O(\log r)$ for $r>2$. It follows that if $f(z)$ has two deficient values $\alpha$ and $\beta$ and if $\kappa=\max (1-\delta(\alpha)$, $1-\delta(\beta))$ then the lower order $\mu$ of $f(z)$ satisfies $\mu \geqq-\log \{\kappa(2-\kappa)\} / \log (1+4 / \kappa(1-\kappa))$. In particular, the lower order of a function which has at least two deficient values must be positive. (Received July 10,1957.)

677. J. M. G. Fell: Weak containment of group representations. Preliminary report.

Let $G$ be a locally compact group. For each $x$ in the group algebra $L_{1}(G)$, let $\|x\|_{c}=\sup \left\|T_{x}\right\|$, where $T$ runs over all unitary representations of $G$. The completion of $L_{1}(G)$ under \|\|$_{c}$ is a $C^{*}$-algebra, called $C^{*}(G)$. A function $\phi$ of the form $\phi(g)=\left(\xi, T_{0} \epsilon \xi\right)$, where $\xi$ is in the space of the representation $T$, is associated with $T$. Now let $W$ be a family of unitary representations of $G$, and $T^{0}$ a unitary representation of $G$. Then $W$ weakly contains $T^{0}$ if each function of positive type associated with $T^{0}$ is a uniform-on-compacta limit of sums of functions of positive type associated with representations in $W$. It is shown that $W$ weakly contains $T^{0}$ if and only if the kernel of $T^{0}$ contains the intersection of the kernels of the $T$ in $W, T^{0}$ and $W$ being considered as acting on $C^{*}(G)$. In particular, the regular representation weakly contains all representations if and only if $C^{*}(G)$ is the completion of $L_{1}(G)$ as an algebra of operators acting on $L_{2}(G)$. This fails for the $2 \times 2$ complex unimodular group; here the regular representation does not weakly contain the supplementary series. (Received July 2, 1957.)

678. Tomlinson Fort: Linear difference and differential equations satisfying conditions at more than one point.

The author establishes sufficient conditions in terms of the nonvanishing of certain determinants of the coefficients that a system of ordinary linear differential equations or a system of ordinary linear difference equations have one and only one solution with prescribed values, some at one point and some at other points. The method of proof is to establish the theorems for difference equations and to pass to the limit for differential equations. (Received June 24, 1957.)

\section{W. C. Fox: Existence of harmonic functions with prescribed singularities.}

Let $R$ be an annulus and let $A$ be a closed disk (in the Riemann surface $X$ ) whose boundary circle is also a boundary curve of $R$. If $\phi$ is harmonic on $R$, then a function $u$ is said to have the singularity $\phi$ on the disk $B=A \cup R$ provided $u$ is harmonic on $X-A$ and $u-\phi$ has a harmonic extension to $B$. If $\left(u_{n}\right)$ is a sequence of functions with the singularity $\phi$ on $B$, and if each $u_{n}$ is bounded on $X-B$ by the maximum of $\left|u_{n}\right|$ on the boundary of $B$, then the sequence is uniformly bounded provided that at some point $x$ of $A$ the values $u_{n}(x)$ all coincide. This fact is instrumental in showing 
that for open $X$ and arbitrary $\phi, R$, and $A$, a function $u$ always exists which has the singularity $\phi$. When $X$ is compact such a $u$ is known to exist iff $\phi$ is the real part of a single-valued function analytic on $R$. For any $X$ such a $u$ exists iff one of the various equivalent extremal problems involving the Dirichlet integral has a solution. (Received July 9, 1957.)

680t. Evelyn Frank: On the equivalence of certain algorithms for the generation of continued fraction expansions.

The equivalence is shown between the author's algorithms [Bull. Amer. Math. Soc. vol. 52 (1946) pp. 144-157, 890-898] and the quotient-difference algorithm of H. Rutishauser [Mitteilungen aus dem Institut für angewandte Mathematik no. 7, Birkhäuser Verlag, Basel, Stuttgart, 1957] for the construction of $J$-fractions and $S$-fractions corresponding to rational functions and to power series. The author's stability criterion is also interpreted in terms of Rutishauser's parameters. (Received July 12, 1957.)

\section{1t. K. O. Friedrichs: Symmetric positive linear differential equa- tions.}

Let $u=\left\{u_{1}, \cdots, u_{k}\right\}$ be a system of functions of the points $x$ of an $m$-dimensional manifold $R$ with boundary $B$. With respect to local coordinate systems $x=\left\{x_{1}, \cdots, x_{m}\right\}$, let $\nabla_{\rho}=\partial / \partial x_{\rho}$, and introduce $k$ by $\kappa$ matrices $\alpha^{\rho}$ and $\kappa$ with the properties I: $\alpha^{\rho}$ is symmetric, $\rho=1, \cdots, m$; II $: \kappa+\kappa^{\prime}$ is positive definite. The differential operator $K=\alpha^{\rho} \nabla_{\rho}+\nabla_{\rho} \alpha^{\rho}+\kappa$ is called "symmetric positive." On $B$ with interior normal $\left\{n_{1}, \cdots, m\right\}$ one finds matrices $\mu$ with III: $\mu+\mu^{\prime}$ non-negative and IV: $u=u_{+}+u_{-}$with $(\mu-\beta) u_{+}=(\mu+\beta) u_{-}=0$. Set $M=\mu+\beta$. Then the boundary value problem $K u=f, M u=0$ with $f \in \Omega_{2}$ has a unique strong solution.- Uniqueness follows from $c\|u\| \leqq\|K u\|$, where $\|u\|^{2}=(u, u)$ is an appropriate integral over $R$. A system of operators $D_{\sigma}=\eta_{\sigma} \nabla_{\rho}+\eta_{\sigma}, \sigma=0, \cdots, s$ is so chosen that $\eta_{\sigma} \eta_{\sigma}^{\rho}=0$ on $B$, and $\left[D_{\sigma}, D_{\tau}\right]$ $=r_{0 \tau}^{\rho} D_{\rho},\left[D_{\sigma}, K\right]=p_{\sigma}^{\tau} D_{\tau}-t_{\sigma} K,\left[D_{\sigma}, M\right]=q_{\sigma} D_{\tau}-t_{\sigma} M$ on $B$. Then $D u=\left\{D_{\sigma} u\right\}$ satisfies an equation $K_{1} D u=D f, M_{1} D u=0$. Assuming $K_{1}$ satisfies $\mathrm{II}_{1}, \mathrm{III}_{1}$ we have a priori $c_{+}\|u\|_{+} \leqq\|K u\|_{+}$, where $\|u\|_{+}^{2}=\sum_{\sigma}\left\|D u_{\sigma}\right\|^{2}$. With the associated negative norm \|\|$-$ the dual inequality $c_{-}\|u\|_{-} \leqq\|K u\|$ holds. Adaptation of the method of P. Lax [Comm. Pure Appl. Math. vol. 8 (1955) pp. 615-633] yields the existence of a solution with $\|u\|_{+}<\infty$. Assumptions $\mathrm{II}_{1}, \mathrm{III}_{1}$ are removed by a continuation argument. Problems of elliptic, hyperbolic, and mixed equations, such as Tricomi's, are included as special cases. (Received May 27, 1957.)

682. R. E. Fullerton: An intersection property for cones in a linear space.

Let $C$ be a cone in a linear space $X$. If $x, y \in X$, let $C^{\prime}=(x+C) \cap(y+C)$. It is shown that if $C^{\prime}$ is itself a cone, there exists an element $z \in X$ such that $C^{\prime}=z+C$. Various examples of cones having the property that there exist points $x, y \in X$ with $(x+C) \cap(y+C)$ a cone are given and discussed. (Received July 11, 1957.)

\section{3t. I. S. Gál: On uniformizable spaces with a unique structure.}

Let $X$ be a uniformizable Hausdorff space. The purpose of this note is to give a n.a.s.c. that $X$ has a unique structure. Let $C(X)$ denote the space of all real valued continuous functions on $X$ topologized by the topology of uniform convergence and let $A(X)$ be the set of real continuous functions on $X$ having compact support. The 
closed sets $C_{1}$ and $C_{2}$ are called normally separable if there exists an $f \in C(X)$ vanishing on $C_{1}$ and identically 1 on $C_{2}$. Theorem: Any two of the following statements are equivalent: (i) $X$ has a unique structure; (ii) if $C_{1}$ and $C_{2}$ are normally separable closed sets in $X$ then at least one of them is compact; (iii) the set $A(X)$ is dense in the space $C(X)$. Condition (ii) shows that compactness is sufficient and local compactness is necessary for uniqueness. Condition (ii) is due to R. Doss [R. Doss, On uniform spaces with a unique structure, Amer. J. Math. vol. 71 (1949) pp. 19-23]. Condition (iii) can be proved without using Doss' result by considering certain ideals in $\left[f^{2}: f \in C(X)\right]$. These are generalizations of filters on $X$. (Received June 28, 1957.)

684t. F. W. Gehring: Asymptotic values for analytic functions of bounded characteristic.

Let $f(z)$ denote a complex-valued function defined in the unit disc $D$ and let $P$ denote a point on the unit circle. We say that $a \in \Gamma(P, f)$ if there exists a Jordan arc $\alpha$ contained, except for $P$, in $D$ such that $f(z) \rightarrow a$ as $z \rightarrow P$ along $\alpha ; a \in \Gamma_{\Delta}(P, f)$ if, in addition, $\alpha$ can be chosen so that it lies in some Stolz angle with vertex at $P$. THEOREM 1. Suppose that $f(z)$ is analytic and has bounded characteristic in $D$. Then $\Gamma(P, f)$ contains at most two finite values and $\Gamma_{\Delta}(P, f)$ contains at most one finite value. THEOREM 2. Suppose that $f(z)$ is analytic and in $H_{p}$ for some $p>0$. Then $\Gamma(P, f)$ contains at most one finite value. THEOREM 3. Suppose that $f(z)$ is analytic and has bounded characteristic in $D$ and that $f(z)$ omits one finite value, say 0 . Then $\Gamma(P, f)$ contains at most one finite value. Moreover, if $\Gamma(P, f)$ contains $a \neq 0, \infty$, it contains only that value. Simple examples show that all these results are best possible. (Received July 12, 1957.)

685t. F. W. Gehring and A. J. Lohwater: On the Lindelof theorem.

Let $D$ denote the unit disc and let $P$ denote a point on the unit circle. The theory of cluster sets is used to derive results, related to a well-known theorem due to Lindelöf [Acta Soc. Sci. fenn. vol. 35, Nr. 4 (1915)], of which the following is typical. THEOREM. Suppose that $f(z)=u(z)+i v(z)$ is analytic and bounded in $D$ and suppose there exist two Jordan arcs $\alpha$ and $\beta$ contained, except for $P$, in $D$ such that $u(z) \rightarrow a$ and $v(z) \rightarrow b$ as $z \rightarrow P$ along $\alpha$ and $\beta$ respectively. Then $f(z) \rightarrow a+i b$ uniformly as $z \rightarrow P$ in each Stolz angle with vertex at $P$. (Received July 12,1957.)

\section{A. W. Goodman: On the critical points of a multivalent function.}

If the critical points of $f(z)$, analytic in $E:(|z|<1)$, are too close to the origin, the function cannot be $p$-valent in $E$. Precisely it is conjectured that if $C_{1}, C_{2}, \cdots, C_{k}(k>p)$ are critical points of $f(z)$, with $\left|C_{j}\right| \leqq\left(2 p-1-2\left(p^{2}-p\right)^{1 / 2}\right)^{1 / k}$ for $j=1,2, \cdots, k$ and if the strict inequality holds for at least one index then $f(z)$ cannot be analytic and $p$-valent in $E$. The bound if true would be sharp as the function $F(z)=z\left(1-z^{k}\right)^{(2 p-2) / k} /\left(1+z^{k}\right)^{2 p / k}$ shows. If $f(z) \neq 0$ in $0<|z|<1$, then the condition $k>p$ can be dropped. Using variational methods properties of the extremal functions for this problem are obtained. A relation between this problem and a coefficient problem for $p$-valent functions is obtained and a new conjecture on the coefficients of $p$-valent functions is suggested. (Received June 28, 1957.)

\section{7t. Hugh Gordon: Integrals defined by abstract $L_{p}$ spaces.}

Let $E$ be a set; let $V$ be the set of all real-valued functions on $E$. Let $V_{0}$ be a linear subspace of $V$ such that: (a) if $f \in V, g \in V_{0}$ and $|f| \leqq|g|$, then $f \in V_{0}$; and (b) if $f_{1}, f_{2}, \ldots \in V_{0}$ and $f_{n} \rightarrow f \in V$ pointwise, then $f \in V_{0}$. Let $L$ be a subspace of the 
Riesz space $V / V_{0}$ and $p \geqq 1$ be a fixed real number such that: (a) $L$ is a Banach lattice; (b) the class of the constant function 1 is in $L$; (c) $\||\bar{f}|\|=\|\bar{f}\|$ for all $\bar{f} \in L$; (d) $\|\bar{f}+\bar{g}\| p \geqq\|\bar{f}\|^{p}+\|\bar{g}\| p$ if $\bar{f}, \bar{g} \in L$ are positive; and (e) $\|\bar{f}+\bar{g}\|^{p} \leqq\|\bar{f}\|^{p}+\|\bar{g}\|^{p}$ if $\bar{f}$, $\bar{g} \in L$ are positive and $\bar{f} \wedge \bar{g}=0$. Then there are a $\sigma$-ring $\mathscr{N}$ of subsets of $E$, a Riesz space $V_{1} \subset V$ and a positive linear functional $F$ on $V_{1}$ with the following properties: (1) $F$ on $V_{1}$ satisfies the theorem of Lebesgue. (If $g, f_{1}, f_{2}, \ldots \in V_{1}, f \in V, M \in \mathbb{T}$, $f_{n}(x) \rightarrow f(x)$ for each $x \in E-M$ and $\left|f_{n}(x)\right|<g(x)$ for each $x \in E-M$ and each $n$; then $f \in V_{1}$ and $F f_{n} \rightarrow F f$.) (Thus $F$ is an integral determined by a $\sigma$-additive measure.) (2) Each element of $L$ is contained in an element of the space $L_{p}$ with respect to $F$ and $V_{1}$, and $L$ and $L_{p}$ are isomorphic, as Banach lattices, in the natural way. Acknowledgement is made to the Nat. Sci. Found. under NSF G 1981. (Received July 12, 1957.)

\section{8t. Hugh Gordon: The spaces $L_{p}$ for generalized integrals.}

Let $\mathfrak{A}$ be a collection of filter bases on a set $E$. We suppose that for each $x \in E$ there is a filter base $Q \in \mathfrak{A}$ such that $x \in A$ for each $A \in \mathbb{Q}$. Let $V$ be the set of all real-valued functions on $E$. Let $f, f_{1}, f_{2}, \cdots \in V$; we write $f_{n} \rightarrow f$ if each filter base $a \in \mathfrak{A}$ contains an $A$ on which $\left\{f_{n}\right\}$ converges to $f$ uniformly. Let $V_{1}$ be a subspace of $V$ and $F$ be a positive linear functional on $V_{1}$ such that: (1) $V_{1}$ is a Riesz space; (2) if $g, f_{1}, f_{2}, \cdots \in V_{1}, f_{n} \rightarrow f$, and $\left|f_{n}\right|<g$ for all $n$, then $f \in V_{1}$ and $F f_{n} \rightarrow F f$; (3) for each $f \in V_{1}$ there are bounded $f_{1}, f_{2}, \cdots \in V_{1}$ such that $f_{n} \rightarrow f$; and (4) $1 \in V_{1}$. (For example, $F$ may be an $Q$-integral; see abstract of $E$. R. Lorch below.) For each real number $p>1$, let $V_{p}$ be the set of $f \in V$ such that $\left(f^{+}\right)^{p},\left(f^{-}\right)^{p} \in V_{1}$. Let $V_{0}$ be the set of $f \in V_{1}$ such that $F|f|=0$. Then $V_{0} \subset V_{p}$ for all $p \geqq 1$; let $L_{p}=V_{p} / V_{0}$. Each $L_{p}$ is a Riesz space and a normed linear space with the norm $\|\bar{f}\|_{p}=\left(F|f|^{p}\right)^{1 / p}$ where $f$ is any representative of the class $\bar{f} \in L_{p}$. If $p, q$ are positive real numbers such that $p^{-1}$ $+q^{-1}=1$ and $\bar{g} \in L_{q}$ is positive, then the mapping $\bar{f} \rightarrow F(f g)$ (where $f, g$ represent $\bar{f}, \bar{g}$ ) is defined and is a bounded positive linear functional on $L_{p}$. Acknowledgement is made to the Nat. Sci. Found. under NSF G 1981. (Received July 12, 1957.)

\section{Felix Haas: $A$ formula relating the index and the sectors of $a$ singular point in $N$-space.}

The index of a nonvanishing vector field, $V$, in $E_{N}$ with respect to a surface, $S$, is defined to be the degree of the map $f: S \rightarrow$ unit sphere which the vector field, $V$, induces. The index of an isolated singular point, $p$, of $V$ is the index of $V$ with respect to sufficiently small spheres enclosing $p$. The index of a smooth closed curve, $C$, is the index of $V$ with respect to sufficiently small tori enclosing $C$. All these definitions reduce to the usual definitions for the plane. If $V$ defines a differential equation with analytic coefficients, the following theorems hold: (1) The index of a singular point $=\left(2+2^{N-2}\left(N_{1}-N_{2}\right)\right) / 2$ where $N$ is the dimension of the space, $N_{1}$ is the number of elliptic sectors, and $N_{2}$ is the number of hyperbolic sectors. (This generalizes a wellknown theorem of Bendixson for the plane.) (2) The index of a closed curve solution is 0 . (3) The index of a closed surface equals the sum of the indices of the singular points inside the surface. (Received July 11, 1957.)

690. J. K. Hale: Sufficient conditions for the existence of periodic solutions of systems of weakly nonlinear first and second order differential equations.

Suppose that a vector function $f(x, t), f=\left(f_{1}, \cdots, f_{n}\right)$, of the real vector 
$x=\left(x_{1}, \cdots, x_{n}\right)$, and the real variable $t$ belongs to the class $A[\omega], \omega>0$, if for every $t,-\infty<t<+\infty$, each component $f_{j}$ of $f$ is analytic in a neighborhood $U$ of $x=(0, \cdots, 0)$ independent of $t$, and the power series expansion of $f_{i}$ in $x_{1}, \cdots, x_{n}$ is convergent in $U$, and its coefficients are periodic functions of $t$ of period $T=2 \pi / \omega$. Consider the system of differential equations (1) $y^{\prime \prime}+D y=\epsilon f\left(y, w, y^{\prime}, \epsilon, t\right)$, $w^{\prime}=\epsilon g\left(y, w, y^{\prime}, \epsilon, t\right)$, where $y=\left(y_{1}, \cdots, y_{\mu}\right), w=\left(w_{1}, \cdots, w_{\nu}\right), D=\operatorname{diag}\left(\sigma_{1}^{2}, \cdots, \sigma_{\mu}^{2}\right)$, $\epsilon, \sigma_{1}, \cdots, \sigma_{\mu}$ are real positive numbers, and $f, g \in A[\omega]$. Let $a=\left(a_{1}, \cdots, a_{\mu}\right)$, $b=\left(b_{1}, \cdots, b_{\mu}\right)$ where each $a_{j}, b_{j}$, is a positive integer, $\sigma=\left(\sigma_{1}, \cdots, \sigma_{\mu}\right)$, $c=\left(c_{1}, \cdots, c_{\mu+\nu}\right)$. By employing a method of successive approximations which has been successively developed by L. Cesari, R. A. Gambill and J. K. Hale it is shown there exist real functions $H_{j}(a, b, c, \sigma, \omega, \epsilon), j=1,2, \cdots, \mu$, analytic for $|\epsilon|<\epsilon_{0}, \epsilon_{0}>0$, such that the following holds: Theorem. If $f\left(y, w,-y^{\prime}, \epsilon,-t\right)=f\left(y, w, y^{\prime}, \epsilon, t\right)$, $g\left(y, w,-t^{\prime}, \epsilon,-t\right)=-g\left(y, w, y^{\prime}, \epsilon, t\right)$ and if, for $|\epsilon|$ sufficiently small, the system of equations (2) $a_{j} \omega / b_{j}+\epsilon H_{j}=\sigma_{j}, j=1,2, \cdots, \mu$, has a solution for some real, nonzero $c, \sigma$ and $\omega$, then (1) has a periodic solution $y(\epsilon, t), \omega(\epsilon, t) \in A\left[\omega / b_{1} \cdots b_{\mu}\right]$ which is even in $t$. Under mild restrictions, there is thus a $\nu$ parameter family of periodic solutions. (Received May 29, 1957.)

691t. J. K. Hale: Linear systems of first and second order differential systems with periodic coefficients.

Consider the vector system of differential equations (1) $u^{\prime \prime}+A u=\lambda f\left(u, v, w, u^{\prime}, v^{\prime}, t\right)$, $v^{\prime \prime}+B v=\lambda g\left(u, v, w, u^{\prime}, v^{\prime}, t\right), w^{\prime}=\lambda h\left(u, v, w, u^{\prime}, v^{\prime}, t\right)$, where $\lambda$ is a real parameter, $u, v, w$ are vectors $A=\operatorname{diag}\left(\sigma_{1}^{2}, \cdots, \sigma_{\mu}^{2}\right), B=\operatorname{diag}\left(\sigma_{\mu+1}^{2}, \cdots, \sigma_{n}^{2}\right)$ and the vector functions $f, g, h$ are linear functions of $u, v, w, u^{\prime}, v^{\prime}$, with coefficients periodic in $t$ of period $2 \pi / \omega, L$-integrable in $[0, T]$ with mean value zero. Further, suppose $\sigma_{j} \neq \sigma_{h}$ $(\bmod \omega i), j \neq h, j, h=1,2, \cdots, n, \sigma_{h} \neq 0(\bmod \omega i) h=1,2, \cdots, n$. By employing a method of successive approximations which has been successively developed by $L$. Cesari, R. A. Gambill and J. K. Hale, the following Theorem is proved: If $f\left(u,-v, w,-u^{\prime}, v^{\prime},-t\right)=f\left(u, v, w, u^{\prime}, v^{\prime}, t\right), g\left(u,-v, w,-u^{\prime}, v^{\prime},-t\right)=-g\left(u, v, w, u^{\prime}, v^{\prime}, t\right)$, $h\left(u,-v, w,-u^{\prime}, v^{\prime},-t\right)=-h\left(u, v, w, u^{\prime}, v^{\prime}, t\right)$, then for $|\lambda|$ sufficiently small all the absolutely continuous solutions of (1) are bounded. (Received May 29, 1957.)

692. P. C. Hammer: Subadditive functions in general. Preliminary report.

The inadequate generality of previous work on subadditive functions is perhaps a result of the subject being considered primarily for limited kinds of applications in analysis. We define subadditivity and reverse subadditivity of functions mapping a set $S$ with a binary operation onto a partially ordered set $G$ with a binary operation. Theorems are proved under as general assumption as possible or expedient. In particular, for example, if $S$ is a semigroup and $G$ a partially ordered group the theory of generating subadditive functions from others is stated in better detail than now given for real functions of a real variable. (Received July 1,1957.)

\section{John Harton, Jr.: Distortion theorems for real star mapping.}

The maximum and minimum values of $\left|f^{\prime}\left(z_{0}\right)\right|$ and $\left|\phi^{\prime}\left(\zeta_{0}\right)\right|$ are studied, where $f(z)=z+a_{2} z^{2}+a_{3} z^{3}+\cdots$ is a simple star mapping of $|z|<1$ for which $a_{2}, a_{3}, \cdots$ are real and $\phi(\zeta)=\zeta+b_{0}+b_{1} / \phi+\cdots$ is a simple star mapping of $|\zeta|>1$ for which $b_{0}, b_{1}, \cdots$ are real. In the interior case the extremal functions map $|z|<1$ onto the $\omega$-plane with one or two radial slits. Explicit formulas are given. In the exterior case, every extremal function must be a function which maps $|\zeta|>1$ onto the $\omega$-plane with 
one-, two-, or three-line segments. In a certain region of $|\zeta|>1$ every extremal function leaves out exactly three line segments and in other regions one or two line segments. These regions and also a region of nonuniqueness where any function of a certain form is an extremal function are given. The methods used are similar to those used by Robinson (Proc. Amer. Math. Soc. vol. 6 (1955) pp. 364-377), and Goodman (Proc. Amer. Math. Soc. vol. 4 (1953) pp. 278-286). (Received July 8, 1957.)

694. L. J. Heider: Measures on Boolean algebras and abstract $(L)$ spaces.

The measures on an arbitrary abstract Boolean algebra are in 1-1 onto, linear, normed-lattice, isomorphic correspondence with the Baire measures on the Stone representation space of the algebra, with countably additive measures corresponding to Baire measures vanishing on Baire sets of the first category while purely finitely additive measures correspond to Baire measures vanishing outside a Baire set of the first category. In consequence, the abstract $(L)$-space of all measures on the algebra is the cross product of the $(L)$-space of countably additive measures with the $(L)$-space of purely finitely additive measures. In particular, the second adjoint space of an abstract $(L)$-space with weak unit is the cross product of the given $(L)$-space with a second newly introduced $(L)$-space. (Received July 8,1957 .)

\section{Henry Helson: Continuous functions as Fourier transforms.}

Given a closed set of points $E$ on the line and a positive weight-function $p(x)$, it is a general problem to determine which continuous functions $F(t)$ defined on $E$ are the restrictions of Fourier transforms of summable functions $f(x)$ satisfying $\int_{-\infty}^{\infty}|f(x)|^{2}$ $\cdot p(x) d x<\infty$. If $E$ is a discrete set $\left\{\lambda_{1}, \lambda_{2}, \cdots\right\}$, the problem requires the determination of the largest and smallest eigenvalues of the infinite matrix $\left(A\left(\lambda_{j}-\lambda_{k}\right)\right)$, where $A(t)$ is the Fourier transform of $1 / p(x)$. Some results have been obtained for particular $p(x)$ and $E$. (Received July 8,1957 .)

\section{C. S. Herz: A theorem on Fourier-Stieltjes transforms.}

Let $\Gamma$ be a locally compact abelian group and $\widehat{\Gamma}$ its character group, - the character of $\Gamma$ corresponding to $t \in \widehat{\Gamma}$ at the point $x \in \Gamma$ is written as $e(t x)$. Let $G$ be a dense subset of $\widehat{\Gamma}$ which is closed under the group operations in $\widehat{\Gamma}$. Further let $\Omega$ be an open subset of $\widehat{\Gamma}, \bar{\Omega}$ its closure. THEOREM 1 . A necessary and sufficient condition that a bounded continuous function, $\lambda$, defined on $\Omega$, be representable in the form $\lambda(t)$ $=\int e(t x) \mu(d x), t \in \Omega$, where $\mu$ is a Borel measure on $\Gamma$ with variation $\leqq M$ is that $\left|\sum c_{i} \lambda\left(t_{i}\right)\right| \leqq M \sup _{x}\left|\sum c_{i} e\left(t_{i} x\right)\right|$ for each finite collection of pairs $\left\{\left(c_{i}, t_{i}\right)\right\}$ where $c_{i}$ is a complex number and $t_{i} \in G \cap \Omega$. THEOREM 2. Let $\phi$ be a continuous function on $\Gamma$ with $\sup _{x}|\phi(x)| \leqq N$. Suppose the spectrum, $\Lambda(\phi)$ is contained in $\Omega$, or more generally that $\phi$ is synthesizable from $\bar{\Omega}$. Then $\phi$ is the limit, uniformly on compact sets, of finite sums $\sum c_{i} e\left(t_{i} x\right)$ where $\sup _{x}\left|\sum c_{i} e\left(t_{i} x\right)\right| \leqq N$ and $t_{i} \in G \cap \Omega$. Theorem 1 generalizes a result of Bochner (Bull. Amer. Math. Soc. vol. 40 (1934) pp. 271-276) where $\Gamma$ is the real line, $\Omega=\widehat{\Gamma}$, and $G$ is the rationals or the reals. (Received July 2, 1957.)

\section{Seizô Itô: $A$ boundary value problem of diffusion equations.}

$D$ denotes a (generally unbounded) domain in $R^{m}$ with its boundary $B$ of $C^{3}$ hypersurface. A diffusion equation $L u \equiv\left(1 / a^{1 / 2}\right)\left(a^{i j} a^{1 / 2} \bar{u}_{j}\right)_{i}+c \bar{u}-\bar{u}_{t}=0$ with mixed-type boundary condition $\left(B_{\phi}\right): \alpha u+\beta u_{n}=\phi$ on $B$, where $u_{i}=\partial u / \partial x^{i}, a=\operatorname{det}\left(a^{i j}\right)^{-1}, a^{i j}$ 's are 
$C^{2}$ and $c$ is nonpositive and Hölder-continuous on $\bar{D}, u_{n}$ denotes normal derivative, $\alpha, \beta \geqq 0$, and $\alpha, \beta$ and $\phi$ are $C^{2}$ on $B$. Results: (1) There exists a function $U(t, x, y)$ satisfying $L U=0$ and $\left(B_{0}\right): \alpha U+\beta U_{n}=0$, in $(t, x)$ for fixed $y$, and also in $(t, y)$ for fixed $x$. (2) Given $f, h$ and $\phi, u(t, x)=\int_{D} U(t, x, y) f(y) d y+\int_{0}^{t} d s \int_{D} U(t-s, x, y) h(y) d y$ $+\int_{0}^{t} d s \int_{D}\left[U(t-s, x, y)-\partial U(t-s, x, y) / \partial n_{y}\right] \phi(y) d y$ satisfies $L u+h=0, u(0, x)=f(x)$ and the boundary condition $\left(B_{\phi}\right)$. (3) If $c$ or $\phi \neq 0$, then $G(x, y)=\int_{0} U(t, x, y) d t$ exists and is locally summable in $x$ and in $y$ separately, and $G(x, y)$ is Green's function of $\left(1 / a^{1 / 2}\right)\left(a^{i j} a^{1 / 2} u_{j}\right)_{i}+c u+h=0$ with the boundary condition $\left(B_{\phi}\right)$. Here $a^{i j}$ is assumed to be positive definite throughout this paper. (Received June 13,1957.)

698t. N. D. Kazarinoff: Complete asymptotic solutions for ordinary second order linear differential equations with two simple turning points. I.

The differential equation $d^{2} u / d z^{2}-\lambda^{2} Q(z, \lambda) u=0, Q(z, \lambda)=\sum_{0}^{\infty} q_{j}(z) \lambda^{-i},|\lambda|>N$, is considered for $z$ in a domain $D$ in which $q_{0}$ has exactly two simple zeros and all the $q_{i}$ 's are analytic. The matter at issue is the behavior of the solutions of this equation for all $z \in D$ as $|\lambda| \rightarrow \infty$. The natural first approximating equation is the modified Weber equation $d^{2} y / d x^{2}-\lambda^{2}\left(x^{2}-1\right) y=0$. The asymptotic behavior of its solutions has been examined by Erdélyi, Kennedy, and McGregor [J. of Rat. Mech. and Anal. vol. 3 (1954) pp. 459-485]. A related equation is constructed by means of a variant of a method due to McKelvey [Trans. Amer. Math. Soc. vol. 79 (1955) pp. 103-123], the construction being based upon the first approximating equation. The asymptotic behavior of solutions of the related equation is studied, and in doing this the results of Erdélyi, Kennedy, and McGregor are refined. The related equation is of the form $d^{2} y / d z^{2}-\left[\lambda^{2} Q(z, \lambda)+\lambda^{-n} R(z, \lambda)\right] y=0$, where $n$ is an arbitrary positive integer and $R(z, \lambda)$ is analytic for $z \in D$ and $|\lambda|>N$. (Received May 31, 1957.)

\section{Bruce Kellogg: Growth of solutions of a certain hyperbolic equa- tion.}

The equation $u_{t t}-\Delta u+u=0$ is considered, where $\Delta$ is the Laplacian in $n$ space variables. An energy integral calculation shows that if the initial data and a number of its derivatives are bounded, then the square integral of the solution and a number of its derivatives over spheres of radius $r$ in the $t=$ constant hyperplanes is $O\left((t+r)^{n}\right)$, and from this it follows that the square of the solution is $O\left(t^{n}\right)$ as $t \rightarrow \infty$. Solutions having this growth are constructed. (Received July 12,1957.)

700t. W. M. Kincaid: Two-point procedures for the numerical solution of systems of equations.

A family of methods has been developed for the numerical solution of a system of equations of the form $f_{i}\left(x_{1}, x_{2}, \cdots, x_{n}\right)=0, i=1,2, \cdots, n$. The methods are iterative, but terminate after a finite number $\left[O\left(n^{2}\right)\right]$ of steps when the equations are linear. They may be regarded as generalizations of the classical method of false position. At each step, a linear function $g$ agreeing with one of the functions $f_{i}$ (or with a linear combination of them) at two points $P$ and $Q$ is constructed, and the point $R$ on the line $P Q$ at which $g=0$ is determined. Another point $S$ not on $P Q$ is then paired with $R$, and the process is repeated with another $f_{i}$. Convergence for nonlinear systems appears to be of the second order in general. The methods are well adapted for use with high-speed digital computers. This research was supported by Department of the Army Contract DA-36-039-SC52634 with the Engineering Research Institute of 
The University of Michigan, operating under a Tri-Service Charter, administered by the U. S. Signal Corps. (Received July 12, 1957.)

\section{1t. Joseph Kist: Locally o-convex spaces. I.}

A locally o-convex space is a partially ordered vector space $E$ together with a locally convex topology for which there exists a fundamental system of neighborhoods of the origin consisting of $o$-convex sets. (A subset $S$ of $E$ is called $o$-convex if it is convex, and if $x \in S$ whenever there exist elements $s, s^{\prime} \in S$ such that $s \geqq x \geqq s^{\prime}$.) The class of locally $o$-convex spaces includes the class of partially ordered normed spaces whose cone of positive elements is a normal cone [cf. M. Krein C.R. (Doklady) Acad. Sci. U.R.S.S. (N.S.) vol. 28 (1940) pp. 13-17]. Several of Krein's results have analogues in the more general setting. As an illustration, let $E$ be a partially ordered locally convex space with closed positive cone $P$. Then, of the following statements, (2) and (3) are equivalent and are implied by (1); if $E$ is a normed space, then all statements are equivalent: (1) $E$ is a locally $o$-convex space. (2) $E^{\prime}=P^{\prime}-P^{\prime}$, where $P^{\prime}$ is the cone of all continuous positive linear functionals (p.l.f.) on $E$. (3) With the weak topology, $E$ is a locally $o$-convex space. The equivalence of (1) and (2) for normed spaces was proved by Krein. (Received July 10, 1957.)

\section{2t. Joseph Kist: Locally o-convex spaces. II.}

It is known [cf. e.g., Proc. Nat. Acad. Sci. U.S.A. vol. 42 (1956) pp. 536-538] that a compatible Banach space topology on a vector lattice is the finest compatible topology. The following result has been obtained (terminology as in the preceding abstract): If a vector lattice $E$ is endowed with a sequentially complete compatible topology $T$, then the following statements are equivalent: (a) $T$ is the finest compatible topology. (b) Any homomorphism (i.e., order preserving linear transformation) of $E$ with range in a locally o-convex space is continuous. (c) Any bounded homomorphism of $E$ with range in a locally o-convex space is continuous. (d) $T=\tau_{\eta}\left(E, E^{\prime}\right)$, and every p.l.f. on $E$ is continuous. $\left(\tau_{\eta}\left(E, E^{\prime}\right)\right.$ is the l.u.b. of all locally $o$-convex topologies which are coarser than $\tau\left(E, E^{\prime}\right)$, the Mackey topology.) (e) $T=\tau_{\eta}\left(E, E^{\prime}\right)$, and every bounded p.l.f. on $E$ is continuous. (f) Every $o$-convex, symmetric, and absorbing subset of $E$ is a neighborhood of the origin. (g) Every $o$-convex and symmetric subset which absorbs all bounded subsets of $E$ is a neighborhood of the origin. (h) $E$ is the $o$-inductive limit of Banach lattices $\left\{E_{\alpha}\right\}$ with respect to homomorphisms $\left\{f_{\alpha}\right\}$ such that $E=\bigcup_{\alpha} f_{\alpha}\left(E_{\alpha}\right)$. (Received July 10,1957.)

703. M. S. Klamkin (p) and D. J. Newman: On the reducibility of some linear differential operators.

The differential equation, $\left[x^{n} D^{2 n}-1\right] y=0$, has been solved previously by Lommel but not in a direct way. By first showing that the operator $x^{n} D^{2 n}$ is reducible to $\left[x D^{2}-(n-1) D\right]^{n}$, the problem is reduced to solving the Bessel equation $\left[x D^{2}-(n-1) D\right] y=a_{r} y$ where $a_{r}^{n}=1$. Whence, $y=x^{n / 2} \sum_{r=0}^{n-1}\left[A_{r} J_{n}\left(2\left(-x a_{r}\right)^{1 / 2}\right)\right.$ $\left.+B_{r} Y_{n}\left(2\left(-x a_{r}\right)^{1 / 2}\right)\right]$. We also effect the reducibility of the following operators: $x^{r n} D^{(r+1) n}, x^{(r+1) n} D^{r n}, D^{(r+1) n} x^{r n}, D^{r n} x^{(r+1) n},\left(x D^{1-r}\right)^{p} D^{2 p r}$, and $x^{2 r p}\left(x^{1-r} D\right)^{p}$. (Received July 9, 1957.)

704. V. L. Klee, Jr.: Extremal structure of convex sets. I.

The Krein-Milman theorem and its converse are extended to certain noncompact convex sets with the aid of the notion of extremal ray, an extremal ray of a convex set $C$ being an open half-line $\rho \subset C$ such that $C \sim \rho$ is convex and the endpoint of $\rho$ is 
an extreme point of $C$. The principal results are as follows: (A) If $C$ is a finitedimensional closed convex set and $C$ contains no line, then $C$ is the convex hull of (ext $C) \cup$ (rext $C$ ), where ext $C$ is the set of all extreme points of $C$ and rext $C$ is the union of all extremal rays of $C$. (B) If $C$ is a locally compact closed convex subset of a locally convex Hausdorff linear space and $C$ contains noline, then $C$ is the closed convex hull of (ext $C$ ) $\cup$ (rext $C$ ). More generally, if $X \subset C$, then cl conv $X=C$ if and only if $\operatorname{cl} X$ ext $C$ and $X$ is asymptotically codirectional with each extremal ray of $C$. (Received April 15, 1957.)

\section{5t. Jacob Korevaar: Local characterization of Fourier transforms.}

Let $(a, b)$ be an interval of length $<2 \pi$. Wiener (The Fourier integral, Cambridge, 1933$, p. 80$)$ proved that a function $f$ defined on $(a, b)$ is the restriction to $(a, b)$ of the Fourier transform of a function $F$ of $L_{1}(-\infty, \infty)$ if and only if $f$ may be given on $(a, b)$ by an absolutely convergent Fourier series $\sum_{-\infty}^{\infty} a_{k} e^{i k t}$. The following generalization is proved. Let $c$ be real and let $1 \leqq \alpha \leqq \infty$. A distribution $\phi$ on $(a, b)$ is the restriction to $(a, b)$ of the Fourier transform of a function $F$ such that $(|t|+1)^{c} F(t) \in L_{\alpha}(-\infty, \infty)$ if and only if $\phi$ may be given on $(a, b)$ by a trigonometric series $\sum a_{k} e^{i k t}$ with the property that the sequence $\left\{(|k|+1)^{c} a_{k}\right\}$ is of class $l_{\alpha}$. Now let $(|t|+1)^{c} F(t) \in L_{\alpha}$, $(|t|+1)^{d} G(t) \in L_{\beta}$ where $c+d \geqq 0, \alpha \geqq 1, \beta \geqq 1, \alpha+\beta \geqq \alpha \beta$. Define tempered distributions $\Phi$ and $\Psi$ by $\Phi=[F]^{(p)}, \Psi=[G]^{(q)}$ where $p$ and $q$ are integers $\geqq 0$. Then the above characterization enables one to prove that $T(\Phi * \Psi)=T \Phi \cdot T \Psi$. Here $\Phi * \Psi=[F * G]^{(p+q)}$ while the product $T \Phi \cdot T \Psi$ is defined locally with the aid of representing trigonometric series. (Received June 17, 1957.)

\section{6t. Jacob Korevaar: Completeness theorems for sets of translates as cancellation theorems.}

Wiener (The Fourier integral, Cambridge, 1933, p. 97) proved that the set of translates $F(t+\lambda),-\infty<\lambda<\infty$, of a function $F \in L_{1}$ is fundamental in $L_{1}$ or complete with respect to $L_{\infty}$ if and only if the Fourier transform $f=T F$ has no (real) zeros. The following proof is based on the preceding note. The set $\{F(t+\lambda)\}$ is complete if and only if $F * G=0, G$ bounded and locally integrable, implies $G=0$. Setting $T G=\psi$ the equation $F * G=0$ is equivalent to $f \cdot \psi=0$. Here $f$ is given locally by an absolutely convergent trigonometric series, $\psi$ by one with bounded coefficients. If $f(t) \neq 0$ everywhere then $f \cdot \psi=0$ implies $\psi=0$ and hence $G=0$. A similar proof may be given for the following theorem of Boas and Bochner (Ann. of Math. vol. 39 (1938) pp. 287-300). Let $C$ denote the Banach space of all continuous functions $F$ on $[-\infty, \infty]$ with the norm $\max |F(t)|, C_{0}$ the subspace of all functions vanishing at $\pm \infty$. The set of translates $\{F(t+\lambda)\}$ of $F \in C$ is fundamental in $C_{0}$ if and only if there is no interval on which the Fourier transform $\phi=T F$ is equal to zero. (Received June 17, 1957.)

\section{7t. Jacob Korevaar: Fourier transforms and the linear harmonic} oscillator.

Let the set $u_{0}, u_{1}, \cdots$ be orthonormal on $(-\infty, \infty)$ and complete with respect to a (large) vector space of functions $C$. Every $f \in C$ is uniquely determined by its expansion $\sum d_{k} u_{k}$ where $d_{k}=\left(f, u_{k}\right)$. One wishes to define a generalized Fourier transformation $[T]$ by the formula $[T] \sum c_{k} u_{k}=\sum c_{k} T u_{k}$. When will the $T u_{k}$ be simple linear combinations of the $u$ 's, for example $T u_{k}=a_{k} u_{k}$ ? Answer: if the $u_{k}$ are eigenfunctions of a boundary value problem $H y=l y$, (B) such that $H$ commutes with Fourier transformation while $T f$ satisfies the boundary condition (B) whenever 
$f$ does. For if $\lambda, u$ is a characteristic pair of such a b.v. problem then so is $\lambda, T u$, and if every eigenvalue $\lambda$ has multiplicity one then $T u=a u$. One is thus led to the b.v. problem of the linear harmonic oscillator $H y \equiv\left(x^{2}-D^{2}\right) y=l y, 0<\int_{-\infty}^{\infty}|y(x)|{ }^{2} d x<\infty$. The paper employs a rigorous version of the factorization method devised by Schrödinger (Proc. Roy. Irish Acad. A vol. 46 (1940) pp. 9-16) to obtain the characteristic pairs in the form $2 k+1, v_{k}(x)=l_{k} N^{k} \exp \left(-x^{2} / 2\right)$. Here $N=x-D$; the $v_{k}$ are the normalized Hermite functions. One has $T v_{k}=(-i)^{k} v_{v_{k}}$. (Received June 17, 1957.)

\section{8t. Jacob Korevaar: Hermite expansions.}

It is shown that the normalized Hermite functions $v_{k}, k=0,1, \cdots$ are complete with respect to the class $A$ of functions $f$ for which there exists a number $a<1 / 2$ such that $f(x) \exp \left(-a x^{2}\right) \in L_{1}$. If $f \in A$ then $\{x\} f \in A$. If $f$ is absolutely continuous and its derivative $D f \in A$ then $f \in A$. If $f$ and $D f$ are absolutely continuous and $D^{2} f \in A$ then $H f=\left(x^{2}-D^{2}\right) f \in A$. The Hermite expansions of $\{x\} f, D f$ and $H f$ are expressed in terms of the expansion coefficients $d_{k}$ of $f$, similarly the expansion coefficients of the Fourier transform $T f$ when $f \in L_{1}$. It is noted that every expansion coefficient of each of these four transforms of $f$ involves only a finite number of $d_{k}$ 's. As an application a simple proof is given of the Fourier inversion theorem in the case where $f$ and $T f$ are in $L_{1}$. The order of magnitude of the expansion coefficients is studied for several classes of functions. For $f$ of at most polynomial growth $d_{k}=O\left(k^{c}\right)$, for $f$ of at most exponential growth $d_{k}=O\left\{\exp \left(c k^{1 / 2}\right)\right\}$, while for $f \in A$ one has $d_{k}=O\left(c^{k}\right)$. (Received June 17, 1957.)

\section{9t. Jacob Korevaar: Pansions.}

A pansion $\phi$ is a formal series $\sum_{k=0}^{\infty} c_{k} v_{k}$ of normalized Hermite functions. If $\phi$ is the expansion of a function $f$ of class $A$ (preceding abstract) or a suitable larger class one identifies $\phi$ with $f$. The global derivative $[D]_{\phi}$, the global product $[x]_{\phi}$ and $[H]_{\phi}$ are defined for all pansions $\phi$ in accordance with the rules valid for the Hermite expansions of the corresponding transforms of functions $f$ of $A$. It is shown that $[D]_{\phi}=0$ if and only if $\phi=\{c\}$, a constant function. Similarly $[x]_{\phi}=0$ if and only if $\phi=c \delta$ where $\delta$ is the Dirac "function" (global derivative of the unit step function). One also defines $[E(D)]_{\phi},[E(x)]_{\phi}$ and $[E(H)]_{\phi}$ where $E$ is an element of a class of entire functions appropriate to $\phi$. The operator $\left[e^{a D}\right]$ plays the role of a translation operator: for every function $f \in A$ and real $a$ one has $\left[e^{a D}\right] f=\{f(x+a)\}$ ("Taylor's formula"). Global multiplication of two pansions is considered, as well as convolution. One has $(2 \pi)^{1 / 2} \delta * \phi=\phi,(2 \pi)^{1 / 2}[D] \delta * \phi=[D] \phi$ for every pansion $\phi$. (Received June 17, 1957.)

\section{0t. Jacob Korevaar: Fourier transforms of pansions.}

Let $\phi$ be the pansion $\sum c_{k} v_{k}$ (preceding abstract). By definition the Fourier transform $[T]_{\phi}$ is the pansion $\sum c_{k} T v_{k}=\sum(-i)^{k} c_{k} v_{k}$. Similarly the conjugate Fourier transform $[\bar{T}]_{\phi}=\sum i^{k} c_{k} v_{k}$. If $\phi$ is a function $f$ of $L_{1}$ then [T] $]_{\phi}$ equals the ordinary Fourier transform $T f$. For pansions the Fourier inversion formula holds without restriction: if $\psi=[T]_{\phi}$ then $[\bar{T}] \psi=\phi$. As an application one has a very simple derivation of Plancherel's theory of Fourier transforms for $L_{2}$. This derivation shows some similarity with Wiener's treatment (The Fourier integral, Cambridge, 1933 , p. 46) based on Hermite expansions, but it is considerably shorter. The following rules are valid without restriction: $[T][D]_{\phi}=i[x][T]_{\phi},[T][x]_{\phi}=i[D][T]_{\phi}$, $[T][H]_{\phi}=[H][T]_{\phi}$. The rules $[T][E(D)]_{\phi}=[E(i x)][T]_{\phi},[T][E(x)]_{\phi}=[E(i D)]$ $\cdot[T]_{\phi}$ are valid for a large class of pansions $\phi$ and entire functions $E$. Finally 
$[T](\phi * \psi)=[T]_{\phi} \cdot[T] \psi$ whenever one of the two sides exists. One has $[T]\{1\}=(2 \pi)^{1 / 2} \delta$. (Received June 17, 1957.)

711t. Jacob Korevaar: Fourier transform theory for pansions of polynomial growth.

Using global properties of the solutions of the differential equation $H y \equiv\left(x^{2}-D^{2}\right) y$ $=f$ it is shown that every function of at most polynomial growth on $(-\infty, \infty)$ can be represented in the form $H^{n} f_{0}$ where $f_{0}$ is a sufficiently differentiable function of $L_{2}$. Pansions of polynomial growth are defined as pansions of the form $\phi=[H]{ }^{n} f_{1}$ where $n \geqq 0, f_{1} \in L_{2}$. The class of these pansions contains all functions of at most polynomial growth and it is closed under Fourier transformation: $[T][H]^{n} f_{1}=[H]^{n} g_{1}$ where $g_{1}=[T] f_{1}$ is also in $L_{2}$. The class is also closed under the operations of global differentiation $[D]$, global multiplication by $[x]$ and translation $\left[e^{a D}\right], a$ real. Every pansion of polynomial growth is a finite order global derivative of a function of at most polynomial growth. Hence these pansions correspond to Laurent Schwartz's tempered distributions. It is finally noted that for a function of polynomial growth $[T] f$ is essentially equal to the global $k t h$ derivative of Bochner's " $k$-Transformierte" of $f$. (Received June 17, 1957.)

712. Jacob Korevaar: Fourier transform theory for pansions of exponential growth.

Using global properties of the solutions of the differential equation $(H+p) y$ $\equiv\left(x^{2}+p-D^{2}\right) y=f(p \geqq 0)$ it is shown that every function of at most exponential growth on $(-\infty, \infty)$ can be represented in the form $E_{0}(H) f_{0}$. Here $E_{0}\left(z^{2}\right)$ $=z^{2} \prod\left(1+z^{2} / p_{j}\right)$ is a special even entire function of exponential type and $f_{0}$ an infinitely differentiable function of $L_{2}$. Pansions of exponential growth are defined as pansions of the form $\phi=[E(H)] f_{1}$ where $E\left(z^{2}\right)$ is any even entire function of exponential type and $f_{1} \in L_{2}$. The class of these pansions contains all functions of at most exponential growth and is closed under Fourier transformation as well as the operations $[G(D)]$ and $[G(x)]$ for every entire function $G$ of exponential type. Every pansion of exponential growth is an infinite order global derivative of exponential type of a function of at most exponential growth. The proof depends on explicit solution of the equation $\phi=[\cos a D] f$. For sufficiently large $a>0$ (depending on the type of $\phi$ ) the equation possesses a function solution $f$ of at most exponential growth. (Received June 17, 1957.)

\section{Stephen Kulik: $A$ method for approximating the imaginary} roots of analytic equations.

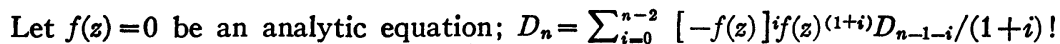
$+[-f(z)]^{n-1} f(z)^{(n)} /(n-1) ! ; Q_{n}=\sum_{i=0}^{n-1}(i)^{n}(u-z)^{n-i} f^{i}(z) D_{n-i}$. Then, if $u$ and $z$ are given arbitrary values such that $\left|\left(u-a_{1}\right) /\left(z-a_{1}\right)\right|>\left|\left(u-a_{i}\right) /\left(z-a_{i}\right)\right|$, where $a_{1}$ and $a_{i}, i=2,3, \cdots$, are the roots of the equation, $\left(u-a_{1}\right) /\left(z-a_{1}\right)=\lim Q_{n} / f(z) Q_{n-1}$, as $n \rightarrow \infty$. If $z=x$ is real, the two real roots adjacent to $x$ can be approximated by using $Q_{n}$ with two different appropriately chosen real values of $u$. If $z=x$ and $u=x+i t$, where $x$ and $t>0$ are real, and the second of two roots, $a+b i$ and $a-b i$, satisfies the above condition, then $a$ and $b$ are found by separating the real and imaginary parts in $Q_{n}=A_{n}+i B_{n}$, namely, $x-a=t f(x) \lim \left\{B_{n-1}\left[f(x) A_{n-1}-A_{n}\right]-A_{n-1}\left[f(x) B_{n-1}-B_{n}\right]\right\}$ - $\left\{\left[f(x) A_{n-1}-A_{n}\right]^{2}+\left[f(x) B_{n-1}-B_{n}\right]^{2}\right\}^{-1}$ and $b=-t f(x) \lim \left\{A_{n-1}\left[f(x) A_{n-1}-A_{n}\right]\right.$ $\left.+B_{n-1}\left[f(x) B_{n-1}-B_{n}\right]\right\} /\left\{\left[f(x) A_{n-1}-A_{n}\right]^{2}+\left[f(x) B_{n-1}-B_{n}\right]^{2}\right\}$, as $n \rightarrow \infty$. The rule for the selection of $u$ and $t$ values in these two cases is simple. (Received June 19,1957.) 
714. R. A. Kunze: An operator theoretic approach to generalized Fourier transforms.

An intrinsic generalization of the Fourier transform is given, based upon the Plancherel theorem and the spectral theorem for normal operators, involving no special kernels or methods of summation, that reduces to the classical transform in the case of $L_{p}(1 \leqq p \leqq 2)$ and at the same time considerably extends the class of functions having transforms. The Fourier transform of a function is, in this approach, again a measurable function, not a distribution or generalized function, and is characterized by its properties as an unbounded operator in $L_{2}$. Specifically, it is shown that corresponding to any measurable function $f$ such that a suitable restriction $L_{f}^{\prime}$ of the operation of convolution by $f$ is essentially normal, there exists a unique measurable function $F$ (the generalized Fourier transform of $f$ ) with the property that the closure $L_{f}$ of $L_{f}^{\prime}$ is unitarily equivalent via the Plancherel transform to multiplication by $F$. A sufficient condition that $L_{f}^{\prime}$ be essentially normal is the existence of a spanning set of functions with compact supports in the domain of $L_{f}^{\prime}$. Many of the functions such as the Heaviside unit function and others commonly used in electrical engineering are shown to have Fourier transforms in this sense. (Received July 11, 1957.)

\section{5t. Joseph Lehner and G. M. Wing: On Weyl's inequality for Eigenvalues.}

It is proved that if $K$ and $Q$ are compact symmetric operators on a Hilbert space to itself, and $Q$ is positive definite, then $\lambda_{n}(K+Q)>\lambda_{n}(K), n=1,2, \cdots$. On the assumption that $Q$ is positive semi-definite, Weyl proved the same statement with ">" replaced by " $\geqq$ " (Göttinger Nachrichten (1911) pp. 110-117). The proof uses the Fischer form of the minimax principle (Monatshefte für Mathematik und Physik vol. 16 (1905) pp. 234-249). (Received July 8, 1957.)

716. H. G. Loomis (p) and I. M. Sheffer: Solution of some linear partial differential equations.

Let $a_{j}(x) \equiv a_{j}\left(x_{1}, \cdots, x_{n}\right)(j=1, \cdots, n)$ be of class $C^{1}$ in some region $R$, with $a_{1}, \cdots, a_{n}$ never simultaneously zero there; and define the operator (1) $\theta f \equiv \sum_{1}^{n} a_{j}(x) \partial f / \partial x_{j}$. Let $\phi(x)$ be a fixed solution of $\theta \phi=1$. To each $f(x) \in C^{1}$ in $R$, associate the function $F(x, t) \equiv F\left(x_{1}, \cdots, x_{n}, t\right)$ satisfying the Cauchy problem (2) $\theta F=0, F\left(x_{1}, \cdots, x_{n}, \phi\right)=f\left(x_{1}, \cdots, x_{n}\right)$. Denote this correspondence by $F \leftrightarrow f$. If $a_{j}, f$ belong to class $C^{(s)}$ then so does $F$. Let $d(x), b_{j}(x) \in C^{1}(j=0,1, \cdots, s-1)$. Every solution of (3) $\theta^{8} f+\sum_{0}^{:-1} b_{j}(x) \theta^{j} f=d(x)$ is the correspondent of some $F$ satisfying the ordinary differential equation (4) $d^{s} F(x, t) / d t^{s}+\sum_{0}^{:-1} B_{j}(x, t) d^{j} F(x, t) / d t^{j}$ $=D(x, t)$ where $D \leftrightarrow d, B_{j} \leftrightarrow b_{j}$; and the Cauchy problem for equation (3) with auxiliary conditions is solved by first solving (4) with suitable auxiliary conditions. The method is also applied to systems of equations in the operator $\theta$. (Received July 11, 1957.)

\section{7t. E. R. Lorch: On integration theory.}

The Daniell program is extended, giving a theory of $Q$-integrals (def. below) in structures that may be devoid of measure. Applications include rotation invariant integrals in infinite dimensional spaces. A projection theorem of the space of continuous functionals onto the manifold of $Q$-integrals is given. Nec. and suff. conditions are given that a multiplicative functional $F$ (extreme functional) be an $Q$-integral. 
These involve the relations of the topological structure of the neighborhoods of $F$ in the space of maximal ideals and the set $Q$. Definition of an $Q$-integral: $E$ is a space of points $x, y, \cdots, \mathfrak{B}$ is (for convenience) a Banach algebra of bounded functions $f(x)$ with uniform norm and containing a unit. $Q$ is a family of sets $A \subset E$. A monotone sequence $\left\{f_{n}\right\}$ of positive functions converges adequately to zero, $f_{n} \downarrow 0$, if $f_{n}(x) \downarrow 0$ uniformly on each $A \in Q$. If $F$ is a continuous positive functional on $\mathscr{B}$, then $F$ is an $Q$-integral if for every $\left\{f_{n}\right\}$ converging adequately to $0, F f_{n} \rightarrow 0$. The two extreme cases are: $\mathcal{Q}$ consists of all finite subsets of $E$ (Lebesgue theory); $Q$ consists of all subsets of $E$ (Riemann theory). Intermediate cases give a spectrum of integrals. The $Q$-integral $F$ can be extended to the class of $Q$-Baire functions and the standard Lebesgue convergence theorem can be established. Parts of this program have been extended by H. Gordon to topological Riesz spaces. (Received July 11, 1957.)

718t. E. H. Luchins: On the concept of the strict radical. Preliminary report.

Call the intersection of those maximal regular right ring (algebra) ideals of a ring (algebra) $A$ which are also left ideals the strict radical of $A$. Call $A$ strictly semisimple (sss) if the strict radical, which contains the Brown-McCoy and Jacobson radicals, is the zero ideal. Examples: A strongly regular ring $R$ (for every $a$ in $R$, there exists $x$ in $R$ such that $a^{2} x=a$ ) is sss; Arens' $B Q^{*}$-algebras (Amer. J. Math. vol. 71 (1949) pp. 763-790) are sss Banach algebras. Let $B$ be a real Banach algebra. Theorems: 1 . The strict radical of $B$ contains the set of topologically nilpotent elements of $B$ and the Jacobson radical of any subalgebra of $B$. 2. $B$ is sss if and only if it is algebraically isomorphic into some $C(X, Q)$, the Banach algebra of all continuous quaternion-valued functions vanishing at infinity on a locally compact Hausdorff space $X$. Corollary: Every subalgebra of a sss real Banach algebra is itself sss. (Received July 12, 1957.)

719t. E. H. Luchins: On continuity of homomorphisms into Banach algebras. Preliminary report.

Let $B$ be a real Banach algebra. Call $B$ absolute if an algebraic homomorphism into $B$ of any real Banach algebra is necessarily continuous. An absolute Banach algebra has a unique Banach norm. Theorem 1: For $B$ to be absolute it is necessary that zero be its only nilpotent element; it is sufficient that $B$ be algebraically isomorphic into some $C(X, Q)$, the Banach algebra of all continuous quaternion-valued functions vanishing at infinity on a locally compact Hausdorff space $X$. An equivalent sufficient condition is that $B$ be strictly semi-simple (see the preceding Abstract). Theorem 2: If $B$ satisfies the descending chain condition on its right ring ideals, then $B$ is absolute if and only if zero is its only nilpotent element. Rickart (Ann. of Math. vol. 51 (1950) pp. 615-628) has shown that if a Banach algebra is semi-simple and has a unique Banach norm, then any algebraic homomorphism of a Banach algebra onto $B$ is necessarily continuous; the present report shows that the resulting proposition is false if the word into is substituted for onto. (Received July 12,1957.)

\section{0t. J. S. MacNerney: Concerning quasi-harmonic operators.}

In a linear normed complete space $S$, let $Q H(S)$ denote the class of quasi-harmonic operators over $S$ (J. Elisha Mitchell Sci. Soc. vol. 71 (1955); Bull. Amer. Math. Soc. Abstract 61-1-99) and $Q \Phi(S)$ the corresponding class of generators, and let the established one-to-one correspondence between $Q H(S)$ and $Q \Phi(S)$ be indicated by the functional notation $M=\mathcal{E}(F)$ for $M$ in the former and $F$ in the latter. THEOREM: 
If each of $F, G$, and $F-G$ is in $Q \Phi(S)$ and $M=\mathcal{E}(F)$ and $N=\mathcal{E}(F-G)$, then the formula $K(u)=\int_{0}^{u} N(0, v) \cdot d G(v) \cdot N(v, 0)$ defines a member $K$ of $Q \Phi(S)$ such that if $P=\mathcal{E}(K)$ then $M(s, t)=N(s, 0) P(s, t) N(0, t)$. Application is made to the problem of reducing systems of Stieltjes mean-integral equations to a canonical "second-order" form. Results will appear in J. Elisha Mitchell Sci. Soc. (Received June 3, 1957.)

\section{Nathaniel Macon (p) and Abraham Spitzbart: Inverses of Vandermonde matrices.}

Recently, the authors obtained explicit formulas for the derivatives of a polynomial $y=f(x)$ of degree $n$ as a linear combination of its values $y_{i}=f\left(x_{i}\right)$ at $n+1$ points defined by $x_{i}=x_{0}+i h,(i=0,1, \cdots, n)$, where the coefficients are expressed in terms of Stirling numbers (to appear in Amer. Math. Monthly). The results are applied here to obtain the elements of the inverse of the matrix whose element in the $i$ th row and $j$ th column is $\left(x_{j-1}\right)^{i-1},(i, j=1,2, \cdots, n+1)$. The differentiation formulas mentioned above are then extended so as to apply to the case in which the $x_{i},(i=0,1, \cdots, n)$, are distinct but otherwise arbitrary, the coefficients of the $y_{i}$ being expressed in terms of the elementary symmetric functions of certain subsets of the $x_{i}$. Finally, this result is used to obtain explicitly the inverse of the general Vandermonde matrix. This last derivation is an alternative to that one would obtain from the classical formula for the values of Vandermonde determinants with missing powers (cf. Polya and Szego, vol. 2, p. 99). (Received July 5, 1957.)

722. J. W. Neuberger: Continuous products and nonlinear integral equations.

This paper deals with the solution of integral equations in an abelian, normed, and complete space $L$, the points of which constitute $S$. Integral equations considered are of the form $Y(t)=A^{+} \int_{c}^{t} d H \cdot Y$, where $A$ is in $S$ and $H$ is a function from the numbers into the collection of all transformations from $S$ into $S$. The continuous product from $c$ to $t$ of $A$ with respect to $H$ is defined as the limit of $\left\{\pi_{R}\left[I^{+} \Delta H\right]\right\} A$ as the mesh of $R \rightarrow 0$. Under certain conditions on $H$, the continuous product from $c$ to $t$ exists for each $t$ in some interval and defines the solution to the above integral equation. If $L$ is Euclidean $n$-space, $H$ can be chosen so that this integral equation is equivalent to a system of $n$ first order nonlinear integral equations. A method of computation for such a system is inherent in the argument. (Received March 6, 1957.)

723t. J. C. C. Nitsche: On the isolated singularities of solutions of $\Delta u=e^{u}$.

Suppose that $u(x, y)$ is solution of an elliptic differential equation, defined and single valued in the neighbourhood of a point $\left(x_{0}, y_{0}\right)$. Without further information one cannot, in general, predict the singular behaviour of $u(x, y)$ when approaching the point $\left(x_{0}, y_{0}\right)$. There are, however, remarkable examples of nonlinear elliptic equations for which this is possible. In case of the minimal surface equation, for example, the singularity must be removable (see L. Bers, Ann. Math. vol. 53). Here the following theorem is proved: Let the function $u(x, y)$ be of class $C^{2}$, and single valued, and satisfy the equation $\Delta u=e^{u}$ in $0<x^{2}+y^{2} \leqq R^{2}$. Then either $u(x, y)$ is regular in $x^{2}+y^{2} \leqq R^{2}$ or it has one of the two forms (i) $u(x, y)=(k-1) \log \left(x^{2}+y^{2}\right)+\phi(x, y)$, $k>0$, (ii) $u(x, y)=-\log \left(x^{2}+y^{2}\right)-2 \log \left|\log \left(x^{2}+y^{2}\right)\right|+\theta(x, y)$. The functions $\phi(x, y)$ and $\theta(x, y)$ are continuous in $x^{2}+y^{2} \leqq R^{2}$ and real analytic in $0<x^{2}+y^{2} \leqq R^{2}$ 
and $D_{\phi}=O\left(r^{2 k-1}\right), D^{2} \phi=O\left(r^{2 k-2}\right), D \theta=O\left(r^{-1}\left|\log ^{-2} r\right|\right), D^{2} \theta=O\left(r^{-2}\left|\log ^{-2} r\right|\right)$. Here $r=\left(x^{2}+y^{2}\right)^{1 / 2}$ and the symbols $D$ and $D^{2}$ mean any first or second order derivatives, resp. (Received June 5, 1957.)

724t. J. C. C. Nitsche: Discontinuities in the solutions of degenerate parabolic equations.

Let $z(x, y)$ be solution of a second order hyperbolic equation. It is possible that $z(x, y)$ belongs to class $C^{k-1}(k \geqq 2)$ but that its derivatives of order $\geqq k$ are only piecewise continuous (cf. Courant-Hilbert, Method. Math. Phys. II, p. 356ff). A curve across which jumps can occur must be a characteristic $C$. The magnitude $\theta$ of the discontinuities satisfies, along $C$, an ordinary differential equation of first order. This is, in general, linear. It may become nonlinear for $k=2$ if the hyperbolic equation itself is nonlinear (cf. Nitsche, J. Rat. Mech. Anal. vol. 2). In the latter case discontinuities, in general, cannot remain bounded and the continuation of a solution across a characteristic, if discontinuities are admitted, may only be possible in a restricted domain. Author shows that this is a situation, also peculiar to certain degenerate parabolic equations (heat equation is not of this type). The equation $z_{x x}=F(x, y, z, p, q)$ is investigated under the assumption that the conic $\alpha^{2}-\alpha \cdot F_{p}^{0}$ $-\beta \cdot F_{q}^{0}=$ const, taken for elements of the solution in points of the $x$-axis, degenerates into a couple of lines. The transport equation for the discontinuities has the form $\theta^{\prime \prime}+a \theta^{\prime}+b \theta+c \theta^{2}=0$. Here $c=0$, however $c \neq 0$ for $k=2$ if $F_{q q}^{0} \neq 0$. (Received June 5, 1957.)

\section{5t. R. R. Phelps: Convex sets and closest points.}

If $A$ is a subset of a normed linear space $E$ and $x, y \in E$ we say that $y$ is pointwise closer to $A$ than is $x$ provided $\|y-a\|<\|x-a\|$ for each $a \in A$. If $x$ is such that no point of $E$ is point-wise closer to $A$ than is $x$, we call $x$ a closest point to $A$. Fejerr [Math. Ann. vol. 85 (1922) pp. 41-48] has noted that in the euclidean plane the set $C(A)$ of all closest points to $A$ is precisely $K(A)$, the closed convex hull of $A$. This result is extended to complete inner product spaces. We say that a normed linear space $E$ has property $(\mathrm{F})$ if for each $A \subset E, C(A) \subset K(A)$. A normed linear space of dimension at least three has property $(F)$ if and only if it is a complete inner product space. In two-dimensional normed linear spaces property $(F)$ is equivalent to strict convexity. If $A$ is a bounded subset of a strictly convex two-dimensional space then $C(A)=K(A)$. The chief tool is a slightly extended version of a theorem of $\mathrm{R}$. C. James [Bull. Amer. Math. Soc. vol. 53 (1947) pp. 559-566, Theorem 4]. (Received July $8,1957$.

726. F. J. Polansky: Completeness and approximation theorems for dilatations of entire functions. Preliminary report.

(1) Let $a_{n} \neq 0, n=0,1,2, \cdots$, and let $f(z)=\sum_{0}^{\infty} a_{k} z^{k}$ be any entire function. The problem investigated here is the completeness of the family $\left\{f\left(\lambda_{n} z\right)\right\}$. Here $\left\{\lambda_{n}\right\}$ is a discrete sequence of complex numbers, the completeness is with respect to the closed linear extension of the set $\left\{z^{n}\right\}_{0}^{\infty}$, the domain of completeness being circles in the $z$ plane. It is shown that any sequence $\left\{\lambda_{n}\right\}$ with a single finite limit point is complete on every circle for any entire function. For the case $\lambda_{n} \rightarrow \infty$, sufficient conditions for completeness are obtained which can be characterized in terms of the MacLaurin series remainder of the function together with products involving the $\lambda_{k}$. (2) The method of (1) depends essentially on approximation of $z^{n}$ by linear combinations of 
the $\left\{f\left(\lambda_{k} z\right)\right\}$. Approximation theorems for functions which are polynomial approximable on arbitrary point sets can be deduced from the method of (1). (Received August 9, 1957.)

\section{Pasquale Porcelli: Uniform completeness and closure of powers} of reciprocals of linear functions.

Let $\left\{k_{p}\right\}_{p-1}^{\infty}$ be set of complex numbers such that $k_{p} \neq 0, k_{p} \notin[-\infty,-1], k_{p} \neq k_{q}$, $p \neq q, p, q=1,2, \cdots, n$ a positive integer, $K_{n}$ the set of functions $\left\{\left(1+k_{p} t\right)^{-n}\right\}_{p=1}^{\infty}$, $0 \leqq t \leqq 1, M\left(K_{n}\right)$ the closed linear manifold generated by $K_{n}$ in $F[0,1](F[0,1]$ denotes the collection of all continuous functions on $[0,1]$ to the complex numbers), and $C_{q}\left(K_{n}\right)$ the closed linear manifold generated by $K_{n}$ in the Lebesgue space $L^{q}[0,1]$. Theorem: If each of $m$ and $n$ is a positive integer and $q \geqq 1$, then each two of the following statements are equivalent: (1) $M\left(K_{n}\right)=F[0,1]$, (2) $M\left(K_{m}\right)=F[0,1]$, (3) $C_{q}\left(K_{n}\right)=L^{q}[0,1]$, (4) $\sum_{p-1}^{\infty}\left(1-\left|\left[\left(1+k_{p}\right)^{1 / 2}-1\right] /\left[\left(1+k_{p}\right)^{1 / 2}+1\right]\right|\right)=\infty$, (5) $1 \in M\left(K_{n}\right),(6) 1 \in C_{q}\left(K_{n}\right),(7)$ there exists a function $h$ in $F[0,1]$ such that $M\left(K_{n}\right)$ is dense in some neighborhood of $h$, and (8) there exists a function $f$ in $L^{q}[0,1]$ such that $C_{Q}\left(K_{n}\right)$ is dense in some neighborhood of $f$. (Received July 12,1957.)

\section{R. T. Prosser: On the general Schrodinger equation.}

This paper considers the General Schrodinger Equation $d U(t) / d t=i \lambda H(t) U(t)$, where $\{H(t)\}$ is a prescribed strongly continuous one-parameter family of operators defined on a suitable Hilbert space, and $\lambda$ a positive constant. We assume (1) For each $t, H(t)$ is defined on a common dense domain $D$, (2) For each $t, H(t)$ admits a self-adjoint extension, (3) For each $\phi \in D$ there is a constant $K$ such that $\left\|H\left(t_{1}\right) H\left(t_{2}\right) \cdots H\left(t_{n}\right) \phi\right\| \leqq K^{n}\|\phi\|, t_{1} \geqq t_{2} \geqq \cdots \geqq t_{n}$. Under these conditions we show that there exists an essentially unique solution $U$ which is unitary and satisfies the usual composition relations. $U$ is given by a power series in $\lambda$ which strongly converges for all values of $\lambda$. Conditions (1) and (2) are necessary; condition (3) is automatic if the $H(t)$ are uniformly bounded, or mutually commute, but some form of (3) is necessary in general. (Received July $12,1957$. )

\section{9t. R. T. Prosser: On the Schrodinger equation of quantum electro- dynamics.}

This paper applies the results of the preceding abstract to the Schrodinger equation which arises in quantum electrodynamics, where $H(t)$ is the total interaction Hamiltonian in the interaction representation and $\lambda$ the charge. Using the canonical particle representations for the electron and photon field operators, we show that this $H(t)$ fails to satisfy condition (2) of the preceding abstract, and hence that the Schrodinger equation admits no proper solution in this case. All of the nontrivial divergences of quantum electrodynamics may be traced to this fact. We show further that if a suitable form factor is introduced into $H(t)$, describing a finite space extension for all electrons, then the conditions of the preceding abstract are all satisfied, and the Schrodinger equation admits a proper solution. We conclude that the divergence difficulties of the present theories are due not to the mathematical methods employed but rather to the physical assumptions made involving the notion of a point particle. (Received July 12, 1957.)

\section{Walter Rudin: On lacunary trigonometric series.}

Define $c_{n}(f)=(1 / 2 \pi) \int_{0}^{2 \pi} f(\theta) e^{-i n \theta} d \theta$. If $1 \leqq r<s<\infty$, let $(r, s)$ denote the class of all sets $E$ whose elements are integers and which have the following property: if $f \in L^{r}$ 
and if $c_{n}(f)=0$ for all integers $n$ not in $E$, then $f \in L^{s}$. The following results are obtained: I. $(r, s)=(1, s)$. II. Suppose $1<p<\infty, 1 / p+1 / q=1$. Then $E \in(1, p)$ if and only if there corresponds to every $f \in L^{q}$ a continuous function $g$ such that $c_{n}(g)=c_{n}(f)$ for all $n \in E$. III. If $E \in(1, p)$ for some $p>1$, then $E$ does not contain arbitrarily long arithmetic progressions. This disproves a conjecture of Sidon (Acta Szeged. vol. 10 (1943) p. 230) to the effect that $E \in(1,2)$ if every term of $E$ is positive and if no term of $E$ is the sum of two terms of $E$. IV. If $E \in(1, q)$ for some $q>2$, then there is a constant $A$ such that no arithmetic progression of $n$ terms contains more than $A \cdot n^{2 / q}$ terms of $E$. V. From IV it follows that there is an $E \in(1,4)$ which is not in $(1, q)$ for any $q>4$. It is conjectured that $(1, r)$ is a proper subset of $(1, q)$ whenever $2<q<r$. V. If $2<q<r$ and if $(1, r)$ is a proper subset of $(1, q)$, then the automorphism group $\Gamma\left(L^{r}\right)$ of the convolution algebra $L^{r}$ is a proper subgroup of $\Gamma\left(L^{q}\right)$. (Received July $3,1957$.

\section{1t. Walter Rudin: Factorization in group algebras.}

The group algebra $L^{1}(G)$ of the locally compact abelian group $G$ is the algebra of all complex-valued functions on $G$ which are Haar integrable, with convolution as multiplication. Theorem: If $G$ is abelian and locally euclidean, then every $f \in L^{1}(G)$ is the convolution of two members of $L^{1}(G)$. That is to say, unrestricted factorization is possible in $L^{1}(G)$; there are no primes. The following lemma is used to established the theorem if $G=R^{n}$ ( $n$-dimensional euclidean space). Lemma: Choose an integer $p>(n+1) / 2$. Suppose $g$ is continuous on $0 \leqq t<\infty, g(t) \rightarrow 0$ as $t \rightarrow \infty$, and $(-1)^{k} \Delta^{k} g(t)>0(k=0, \cdots, p ; 0<t<\infty)$, where $\Delta g=t^{-1}(d g / d t), \Delta^{k} g=\Delta\left(\Delta^{k-1} g\right)$. Define $h$ on $R^{n}$ by: $h(x)=g(|x|)$, where $|x|$ is the distance from $x$ to the origin. Then $h$ is the Fourier transform of some $f \in L^{1}\left(R^{n}\right)$. Once the theorem is proved for $G=R^{n}$, the general case follows from known facts about the structure of locally euclidean abelian groups. (Received July 3, 1957.)

\section{2t. Walter Rudin: Discrete flows on compact spaces.}

Let $C(X)$ be the set of all continuous real functions on $X$. The following is a known consequence of the individual ergodic theorem: If $X$ is a compact metric space and if $h$ is a homeomorphism of $X$ onto $X$, then there exists a point $p \in X$ at which the sequence $(1 / n) \sum_{1}^{n} f\left(h^{i}(p)\right)$ converges for every $f \in C(X)$, as $n \rightarrow \infty$. Jerison (Canadian J. Math. vol. 9 (1957) p. 83) conjectures that the same is true if $X$ is the (nonmetric) compact space $\beta N-N$ (where $N$ is the set of all positive integers, with the discrete topology, and $\beta N$ is the Čech compactification of $N$ ) and if $h$ is the homeomorphism of $X$ induced by the mapping $k \rightarrow k+1$ of $N$ into $N$. Since there are no finite orbits under this homeomorphism, the conjecture is disproved by the following theorem, which is the main result of the present paper: If $\left\{p_{i}\right\}$ is a simple countable sequence of points in $\beta N$, such that $p_{i} \neq p_{j}$ if $i \neq j$, then the sequence $(1 / n) \sum_{1}^{n} f\left(p_{i}\right)$ fails to converge, as $n \rightarrow \infty$, for some $f \in C(\beta N)$. (Received July 3, 1957.)

\section{3t. Diran Sarafyan: Determination of singular points of first order} ordinary differential equations through the use of integrating factors.

In a previous work (Bull. Amer. Math. Soc. Abstract 63-4-529) (534th meeting of AMS) the author established results which can be crystallized in the following Theorem I: The exact differential equation $M d x+N d y=0$ has no singular points in a region $R$ where $M, N$ and $f(x, y)=-M / N$ are continuous. Outside of $R$ they are determined by $1 / M=0$ and/or $1 / N=0$ on condition that at such points $M$ and $N$ 
are continuous. Although this paper creates a new method for the determination of the singular points of $y^{\prime}=f(x, y)$ through the use of its integrating factors, it may be considered as the extension and enlargement of the above mentioned work, and concludes with a generalized theorem which covers exact as well as non exact differential equations. Let $\lambda=\lambda^{\prime}(x, y)$ be an integrating factor for $M d x+N d y=0$. Since $\lambda M d x+\lambda N d y=0$ is an exact differential equation, the application of the above Theorem I will give the following generalized Theorem II: The differential equation $M d x+N d y=0$ has no singular points in a region $R$ where $\lambda M, \lambda N$ and $f(x, y)=-M / N$ are continuous, $\lambda$ being an integrating factor. Outside of the region $R$ the singular points are determined by $1 /(\lambda M)=0$ and/or $1 /(\lambda N)=0$, on condition that at such points $\lambda M$ and $\lambda N$ are continuous. (Received July 15, 1957.)

\section{4t. J. C. Scanlon: A note to Poincarê's perturbation method.}

Following K. O. Friedrichs' treatment [Advanced ordinary differential equations (mimeographed notes) ], a study is made of the periodic solutions of a nonautonomous system of differential equations $d x / d t=F(x, t, \epsilon)$, where $x$ and $F$ are $n$-vectors and $F$ satisfies appropriate differentiability and periodicity conditions, by using Poincaré's perturbation method. The results are then extended by using topological degree. The technique yields new existence theorems if the variational equation has at least two solutions, i.e., if the degree of degeneracy exceeds one. This is usually the case in problems of mechanical or electrical oscillations. (Received May 13, 1957.)

\section{J. A. Schatz and Albert Wilansky (p): Depressing subspaces of a conjugate Banach space.}

For a Banach space $X$, let $L$ be a norm-closed proper linear subspace of $X^{*}$ which is total over $X$ i.e. $f(x)=0$ for $f \in L$ implies $x=0$. (Then $X$ cannot be reflexive). Let $\|x\|_{L}$ be the norm of $x \in X^{* *}$ as a function on $L$. (a) It can happen that $\|x\|_{L}<\|x\|$ for some $x \in X$. Then although $L$ is weak ${ }^{*}$ dense in $X^{*}, L \cap B$ is not weak ${ }^{*}$ dense in $B$ ( $B=$ unit ball). (b) For some $y \in X^{* *} \sim X, y^{\perp}$ has property (a); for others it does not. Call $L$ depressing if $\|x\|_{L}$ is not equivalent to the norm of $X$ i.e. given $\epsilon>0$, $\|x\|_{L}<\epsilon\|x\|$ for some $x$. For $y \in X^{* *}, y^{\perp}$ is never depressing. (Compare (b)). THEOREM. $L$ is depressing iff $L^{\perp}+X$ is not norm-closed in $X^{* *}$. Since $L^{\perp}$ and $X$ are disjoint this means $L^{\perp}$ and $X$ are "far from orthogonal" (Kober, Lorch). Examples of nonclosed $A+B$ are known. We give one in which $A=L^{\perp}, B=X$. Hence THEOREM. There exists a depressing subspace. (Received July 10,1957.)

\section{V. L. Shapiro: Upper and lower divergence of vector fields.}

Using the notation $X=(x, u)$ and $C(X, t)=$ circumference of the circle with radius $t$ and center $X_{0}$, and letting $V(X)=[A(X), B(X)]$ be a continuous vector field in the neighborhood of the point $X_{0}$, define $\operatorname{div}^{*} V\left(X_{0}\right)=\lim \sup _{t \rightarrow 0}\left(\pi t^{2}\right)^{-1}$ - $\int_{C\left(x_{0}, t\right)} A d y-B d x$. Define $\operatorname{div} * V\left(X_{0}\right)$ similarly using lim inf. If $\operatorname{div} V_{*}\left(X_{0}\right)=\operatorname{div}^{*} V\left(X_{0}\right)$ is finite, call this common value $\operatorname{div} V\left(X_{0}\right)$. In this paper, the following theorem is proved: Theorem. Let $D$ be a bounded domain in the plane and let $E$ be a closed set of capacity zero contained in $D$. Suppose (i) $V(X)=[A(X), B(X)]$ is a continuous vector field defined in $D$. (ii) $\operatorname{div}^{*} V(X)$ and $\operatorname{div}_{*} V(X)$ are finite in $D-E$. (iii) $\operatorname{div}_{*} V(X)$ $\geqq F(X)$ for $X$ in $D$ where $F(X)$ is Lebesgue integrable on $D$. Then div $V(X)$ is defined almost everywhere in $D$ and is Lebesgue integrable on every closed subdomain of $D$. Furthermore if $R$ is closed subdomain of $D$ bounded by a simple closed rectifiable curve $C$, then $\int_{C} A d y-B d x=\int_{R} \operatorname{div} V(X) d X$. This theorem can be extended to $n$ - 
dimensional Euclidean space, $n \geqq 2$, if due consideration is paid to the boundary surface of $R$. (Received April 17, 1957.)

\section{7t. V. L. Shapiro: Negligible sets for the divergence theorem.}

Let $D$ be a bounded domain in the plane with a simple closed rectifiable curve $C$ as its boundary and let $Z$ be a closed set of Lebesgue measure zero contained in $D$. With respect to this set $Z$, define a class of vector fields $C_{Z}$ as follows: $V(X)$ is in $C_{Z}$ if (1) $V(X)$ is continuous in $D+C-Z$. (2) $V(X)$ is in $L^{2}$ on $D$. (3) $\operatorname{div} * V(X)$ and $\operatorname{div}^{*} V(X)$ are finite in $D-Z$. (4) div $V(X)$ exists almost everywhere in $D$ and is in $L^{1}$ on $D$. (The notation of the preceding abstract Upper and lower divergence of vector fields is being used.) $Z$ is then said to be a negligible set for the divergence theorem if $V$ is in $C_{Z}$ implies that the divergence theorem holds for $V$ with respect to $D$, i.e. $\int_{C} A(X) d y-B(X) d x=\int_{D} \operatorname{div} V(X) d X$. The following theorem is then proved: A necessary and sufficient condition that $Z$ be a negligible set for the divergence theorem is that $Z$ be of logarithmic capacity zero. Condition (2) in the definition of class $C_{Z}$ is necessary if one wants $Z$ to be different from the empty set as the vector field $V(X)=$ grad $\log |X|$ illustrates. The above theorem can be extended to $n$-dimensional Euclidean space, $n \geqq 2$, if due consideration is paid to the boundary of the domain $D$. (Received July 5, 1957.)

\section{Harold Shniad: Bieberbach's conjecture for schlicht functions.}

For functions which are analytic and schlicht in the unit circle and which have the usual normalization, Bieberbach has conjectured that the $n$th coefficient is at most $n$. In the present paper this conjecture is verified up to and including $n=6$. The basic tools used are a combination of various types of variations on Loewner's function $k$ and a representation theorem which characterizes functions whose real part is non-positive in the unit circle. The method can be used for higher values of $n$, but the calculations required would be of considerable length. (Received July 10, 1957.)

\section{W. F. Stinespring: An element of the enveloping algebra of a} Lie group.

Let $G$ be a Lie group which is unimodular. Let $X_{1}, \cdots, X_{n}$ be any basis for the right invariant Lie algebra of $G$. Let $p=[n / 2]+1$. Then $\left(1-X_{1}^{2}-\cdots-X_{n}^{2}\right)^{p}$ acting on $C_{0}^{\infty}(G)$, the infinitely differentiable functions with compact support on $G$, has an extension $D$ to a self-adjoint operator on $L_{2}(G)$ that commutes with right translations such that $D^{-1}$ is left convolution by a positive, positive-definite function in $L_{1}(G)$. The operator $D^{-1}$ is integrable with respect to the dual gage space of $G$. Let $P$ be any subrepresentation of the regular representation of $G$, and let $t$ be the gage on the algebra of $P$ induced by the dual gage of $G$. If $f$ is in $C_{0}^{\infty}(G)$, then $P_{f}=\int f(x) P(x) d x$ is such $\left|t\left(P_{f}\right)\right| \leqq\left\|D^{-1}\right\|_{1}\|D f\|_{1}$. Therefore, $\chi_{p}(f)=t\left(P_{f}\right)=\int(D f)(x) k(x) d x$ for some $k$ in $L_{\infty}(G)$. In other words, the "character" $\chi_{P}$ of $P$ is a $2 p$-fold distribution derivative of a bounded measurable function. (Received July 12, 1957.)

740. I. H. Sublette: On a question raised by J. Schur. Preliminary report.

J. Schur studied the properties of power series $f_{n}(z)$ which are analytic and have a modulus not greater than unity in $|z|<1$ [Journal für Reine und Angewandte Mathematik, vol. 147 (1917) pp. 205-232; also vol. 148 (1918) pp. 122-145]. By means of a 
special algorithm he generated an infinite sequence of bounded functions $\left\{f_{n}\right\}$ $(n=0,1,2, \cdots)$. He showed that if the series $\sum f_{n}(0)$ converges absolutely, $f_{0}$ is continuous in the set $|z| \leqq 1$ and its upper bound there is less than unity. He raised, but did not answer, the following question: do the above conditions on $f_{0}$ imply the absolute convergence of the series $\sum f_{n}(0)$ ? A recent investigation into electrical network theory by the present author has produced the following results for the special case of rational $f_{0}$ : (a) if $\left|f_{0}\right|$ is not equal to unity everywhere on $|z|=1$, $\lim _{n \rightarrow \infty} f_{n}(0)=0$; (b) if $\left|f_{0}\right|<1$ everywhere on $|z|=1$, the series $\sum f_{n}(0)$ converges absolutely. (Received June 20, 1957.)

741. A. H. Van Tuyl: A property of some nonlinear sequence transformations.

Let $A_{n}$ be a convergent complex sequence such that $\Delta A_{n+1} / \Delta A_{n}=\sum_{i=0}^{\infty} a_{i} n^{-i}$, where $\Delta A_{n}=A_{n+1}-A_{n},\left|a_{0}\right|<1$, and the $a_{i}$ are independent of $n$ and not all zero for $i \geqq 1$. Let $e_{k}\left(A_{n}\right)$ be the $k$ th order transformation of $A_{n}$ defined by D. Shanks (Journ. Math. Phys. vol. 34 (1955) pp. 1-42), let $e_{k}^{m}\left(A_{n}\right)=e_{k}\left(e_{k}^{m-1}\left(A_{n}\right)\right), m=1,2, \cdots$, with $e_{k}^{0}\left(A_{n}\right)=A_{n}$, and let $e_{k_{1}}^{m_{1}} e_{k_{2}}^{m_{2}}\left(A_{n}\right)=e_{k_{1}}^{m_{1}}\left(e_{k_{2}}^{m_{2}}\left(A_{n}\right)\right)$. Then if $A_{n}$ and $B_{n}$ have the same limit, and $\Delta B_{n} / \Delta A_{n}=n^{-z l} \sum_{i=0}^{\infty} c_{i} n^{-i}$, where $l$ is an integer $\geqq 1$ and $c_{0} \neq 0$, it follows that $B_{n}=e_{k_{1}}^{m_{1}} \cdots e_{k_{r}}^{m_{r}}\left(A_{n}\right)+\alpha_{n}\left(k_{1}, \cdots, k_{r} ; m_{1}, \cdots, m_{r}\right) \Delta e_{k_{1}}^{m_{1}} \cdots e_{k_{r}}^{m_{r}}\left(A_{n}\right)$, where the $k_{i}$ and $m_{i}$ are any positive integers such that $m_{1} k_{1}+\cdots+m_{r} k_{r}=l$, and $\alpha_{n}\left(k_{1}, \cdots, k_{r} ; m_{1}, \cdots, m_{r}\right)$ tends to a finite limit as $n \rightarrow \infty$. In general, two transformations of the form $e_{k_{1}}^{m_{1}} \cdots e_{k_{r}}^{m_{r}}\left(A_{n}\right)$, where $m_{1} k_{1}+\cdots+m_{r} k_{r}=l$, converge with the same order of rapidity when $A_{n}$ is of the class considered. However, for special values of $a_{0}, a_{1}$, and $k$, the rates of convergence are different. Further transformations $e_{j, k}\left(A_{n}\right)$ are defined for $j=1,2, \cdots$ which accelerate the convergence of certain sequences $A_{n}$ for which $\Delta A_{n+1} / \Delta A_{n}=1-1 / n-1 /(n \log n)-\cdots$ $-s /\left(n \log n \log _{2} n \cdots \log _{j-1} n\right)+\sigma_{n} / n^{2}$, where Res $>1, \log _{i} n$ is the $i$-times iterated logarithm of $n$, and $\sigma_{n}$ tends to a finite limit as $n \rightarrow \infty$. Results similar to the preceding hold for a given $j \geqq 1$. (Received July 15,1957 .)

\section{2t. John Wermer: Rings of analytic functions.}

Let $D$ be a finite region on a Riemann surface whose boundary is a simple closed analytic curve $\Gamma$. Denote by $R(D)$ the ring of all functions analytic on $D \cup \Gamma$. Let $A$ be a subring of $R(D)$ which contains the constants. In order that $A$ be uniformly dense in $R(D)$ on $D \cup_{\Gamma}$ it is clearly necessary that: (i) $A$ separates points on $D \cup_{\Gamma}$, and (ii) If $p \in D$, there is some $f p$ in $A$ which is locally schlicht at $p$. Theorem: Assume (i) and (ii) and also assume that there is some $\phi$ in $A$ which is locally schlicht at each point of $\Gamma$. Then $A$ is uniformly dense in $R(D)$ on $D \cup \Gamma$. (Received July 11, 1957.)

\section{3t. John Wermer: Polynomial approximation on curves.}

Let $\Gamma$ be a simple closed Jordan curve in the space of $n$ complex variables $Z_{1} \cdots Z_{n}$. By the $h u l l$ of $\Gamma, h(\Gamma)$, we mean the set of all points $\left(Z_{1}^{0}, \cdots, Z_{n}^{0}\right)$ such that for all polynomials $P$ in $n$ variables: $\left|P\left(Z_{1}^{0}, \cdots, Z_{n}^{0}\right)\right| \leqq \max \left|P\left(Z_{1}, \cdots Z_{n}\right)\right|$ taken over $\Gamma$. It is trivial that $h(\Gamma)$ is a compact set containing $\Gamma$. Also, it is easy to give examples of curves $\Gamma$ with $h(\Gamma)=\Gamma$. Theorem 1: Assume $\Gamma$ admits an analytic parametrisation $Z_{i}=\phi_{i}(u),|u|=1, i=1, \cdots n$, where each $\phi_{i}$ is analytic on the circle $|u|=1$. Assume also $\phi_{1}^{1}(u) \neq 0$ for $|u|=1$. Then $h(\Gamma)-\Gamma$ is either empty or is a complex analytic variety of one complex dimension with at most finitely many singularities. Theorem 2: Let $T$ be a Jordan arc in the space of $n$ variables and let $T$ admit an analytic parametrisation $Z_{i}=\psi_{i}(t), 0 \leqq t \leqq 1, i=1, \cdots n$, and $\psi_{1}^{1}(t) \neq 0,0 \leqq t \leqq 1$. Then every 
continuous function on $T$ is uniformly approximable on $T$ by polynomials in $Z_{1}, \cdots, Z_{n}$. (Received July $11,1957$. )

\section{Harold Widom: Equations of Wiener-Hopf type.}

A general procedure is given for solving homogeneous integral equations of Wiener-Hopf type $\left(^{*}\right) \int_{0}^{\infty} k(x-y) f(y) d y=f(x)$, where the only growth restriction on the kernel is $k(x) \in L_{2}(-\infty, \infty)$. It is seen that the number and general nature of the solutions of $\left(^{*}\right)$ are determined by the behavior of $K(\xi)=\int_{-\infty}^{\infty} e^{i \xi x} k(x) d x$ near the zeros of $1-K(\xi)$. For example, if $1-K(\xi)$ has finitely many zeros $\alpha$ possessing multiplicities $m_{\alpha}$, the solutions of $\left(^{*}\right)$ (under certain additional assumptions) are all of the form $\sum_{\alpha} Q_{\alpha}(x) e^{i \alpha x}+g(x)$ where each $Q_{\alpha}(x)$ is a polynomial of degree $<m_{\alpha}$ and $g(x) \in L_{2}(0, \infty)$; if $K(\xi)$ is real, there are exactly $\sum m_{\alpha} / 2$ linearly independent solutions of this form. Again, if $K(\xi)$ is real, $1-K(\xi)=0$ at $\xi=0$ and nowhere else and $|\xi|^{-\sigma}(1-K(\xi))$ is bounded and bounded away from zero near $\xi=0$, then (again under certain additional assumptions) $r$ independent solutions of $\left(^{*}\right)$ are obtained, where $r=\sigma / 2$ if this is an integer and $r=[\sigma / 2]+1$ otherwise. (Received July 2, 1957.)

\section{Hidehiko Yamabe: $A$ kernel function of a parabolic differential equation.}

$D$ denotes an open domain with smooth boundary in a $d$-dimensional euclidean $R$. Given two integrable functions $\phi$ and $\psi$ over $R \times R,(\phi * \psi)(x, y)=\int_{D} \phi(x, z) \psi(z, y) d z$. $E_{t}(x, y)$ denotes the kernel function $\left(2(\pi t)^{1 / 2}\right)^{-d} \exp (-|x-y| / 4 t)$ of an equation $\partial U / \partial t=\Delta U$. Set $\left(E_{t / n} *\right)^{n}(x, y)=\left(E_{t / n} *(n) * E_{t / n}(x, y)\right.$. Then $\lim _{n}\left(E_{t / n} *\right)^{n}(x, y)$ $=K(x, y ; t)$ exists and coincides with the kernel function of an equation $\partial U / \partial t=\Delta U$ with 0 boundary. Here $\Delta$ denotes the ordinary Laplacian. (Received June 13,1957.)

\section{Applied Mathematics}

\section{6t. R. W. Bass: Stability of nonlinear control systems (I).}

Notations: Bull. Amer. Math. Soc. Abstracts 63-3-359 through 63-3-361. Let $S$ represent a control system, which is "stable" when the rest point $x_{0}$ is globally asymptotically stable. A NASC for this is criterion (iv), Abstract 361 . By strengthening (iv) we attain a sufficient criterion, easier to apply in specific cases than Lyapunov's Direct Method, and of wide usefulness. Let $P^{\prime}$ denote $P$ transposed, and curl $g$, the skew-symmetrized Jacobian matrix. Theorem. $S$ is stable if there exists a continuous matrix $P(x)$ such that in $E^{n}$ (i) the tensor curl $P(x) f(x) \equiv 0$; (ii) $P(x)$ is positive definite for all $x \neq 0$; (iii) $P^{\prime}(x) x \cdot f(x) \leqq-\epsilon\|x\|$ for some $\epsilon>0$ and all $\|x\| \geqq 1$. Corollaries. Routh-Hurwitz linear stability criteria; (b) criteria of Ayzerman, Erugin, Malkin; Barbasin, Simanov; and Cartwright for nonlinear systems of second, third, and fourth order; (c) general criteria ( $n$th order, nonlinear) of Hahn, Zubov, Krasovskii, and Kalman; (d) all general criteria for controls given in the books of Lur'e and Letov; (e) (after modifications) stability criteria for "pre-endpoint motion" of discontinuous systems, such as studied by e.g. Flügge-Lotz, and André and Seibert. (Received July 12, 1957.)

\section{E. K. Blum: On the Runge-Kutta fourth-order method.}

Consider the system of differential equations, $y^{\prime}=f(x, y)$, with initial value $y_{0}$ at $x_{0}$. The numerical solution of this initial value problem by the Runge-Kutta fourth order method takes the form, $y_{n+1}=y_{n}+\left(K_{1}+2 K_{2}+2 K_{3}+K_{4}\right) / 6$, where $K_{1}=h f\left(x_{n}, y_{n}\right)$, 
$K_{2}=h f\left(x_{n}+h / 2, y_{n}+K_{1} / 2\right), K_{3}=h f\left(x_{n}+h / 2, y_{n}+K_{2} / 2\right), K_{4}=h f\left(x_{n}+h, y_{n}+K_{3}\right) . \mathrm{A}$ variation of this method was proposed by S. Gill [Proc. Cambridge Philos. Soc. vol. 47 (1950) pp. 96-108]. The two advantages claimed for the Gill modification are: (1) In automatic computers, it requires $3 K+B$ storage registers whereas the RungeKutta formulas require $4 K+B$, where $K$ is the order of the differential system; (2) The computation can be arranged in such a manner that rounding errors are reduced appreciably. In the present paper, it is shown that (1) the Runge-Kutta formulas can also be arranged so that the process requires $3 K+B$ storage registers, and (2) the ingenious techniques employed by Gill to reduce rounding error can be applied to the rearranged Runge-Kutta formulas with equal effectiveness. All constants in this modification are rational, whereas in the Gill version there are irrational constants. (Received July 12, 1957.)

748. Ward Cheney and A. A. Goldstein (p): A finite algorithm for the linear inequality and Tchebycheff problems.

The problem of solving a system of linear inequalities and the Tchebycheff problem for an inconsistent system of linear equations reduce to the problem of minimizing (or making negative) the function $F(x)=\max _{i}\left[\left(A^{i}, x\right)-b_{i}\right]$ where $A^{i} \in E_{n}, x \in E_{n}$, $b \in E_{m}, i=1, \cdots, m$. An algorithm is given for accomplishing this, but a simpler version adequate for the usual case in practice will be outlined here. Define residuals $R^{i}(x)=\left(A^{i}, x\right)-b_{i}$. Let $x^{0}$ be a first approximation. Suppose that $F\left(x^{0}\right)=R^{1}\left(x^{0}\right)$ $>R^{i}\left(x^{0}\right), i \neq 1$. The minimum of $F$ on the ray of steepest descent $x^{0}-\lambda A^{1}$ is attained at a point $x^{1}$ which induces two maximum residuals, say $R^{1}\left(x^{1}\right)$ and $R^{2}\left(x^{1}\right)$. The relation $R^{1}=R^{2}$ serves to eliminate a variable from the original system and the process may be repeated in the reduced system. After $n-1$ eliminations, $F(x)$ becomes a function of one variable in the reduced system. At a minimum point in this system the eliminated variables are restored and this results in a vector inducing $n+1$ equal maximum residuals in the original system. A method is given for determining a ray $D$ of descent by solving an $n \times(n+1)$ system of inequalities. $F(x)$ is minimized on $D$ and the entire process may begin anew. The set of solution vectors is described. (Received June 24, 1957.)

749t. Nathanael Coburn: $A$ class of steady supersonic rotational axial symmetric flows.

In this paper, we study a class of rotational axial symmetric supersonic flows which possess a family of $\infty^{1}$ parallel planes as characteristic surfaces. By use of the theory of a previous paper [Intrinsic form of the characteristic relations in the steady supersonic flow of a compressible fluid, Quarterly of Applied Math., forthcoming], the basic equations are obtained for the case, $a_{23}=0$, of that paper. This case leads to a set of three partial differential equations for $M$, the Mach number, $h_{0}$, the stagnation enthalpy, and $r$, the radial distance, in terms of $z$, the distance along the axis of the flow, and another variable. Two of these equations are linear. For any proper choice of $M$ as a function of $h_{0}, r$, the linear equations can be solved to determine $h_{0}$ as a function of $z$ and $r$. The case $M=M(r)$ is treated in detail. (Received July 5,1957.)

750t. Nathanael Coburn: The characteristic relations in the nonsteady flow of a compressible gas. Preliminary report.

Since the Newtonian mechanics of continuous media is based on three space variables, with time playing the role of an independent parameter, it is very difficult to find the geometric relations between the velocity vector, the bicharacteristic and 
normal cones for the nonsteady flow of a compressible gas. Hence, the space-time metrics of special and general relativity are introduced and the theory of characteristic systems [for the steady case, see Characteristic relations for the steady flow of a compressible fluid, C. L. Dolph and N. Coburn, Proc. of the First Symposium of Applied Math., McGraw-Hill, New York, 1949, pp. 55-66] is studied. The partial differential equation satisfied by the characteristic manifolds is determined and it is shown that the velocity four-vector, a normal to a characteristic manifold, and the corresponding bicharacteristic are related in a similar manner to the steady case. The return to Newtonian mechanics is made via the usual first approximation. A complete characteristic system is yet to be found. (Received July 5,1957 .)

751t. R. T. Dames: Stability and convergence for a numerical solution of the Goursat problem.

This paper considers the notions of stability and convergence as applied to the numerical solution of systems of second order hyperbolic equations expressed in characteristic coordinates. Emphasis is placed on linear equations in two independent variables and boundary conditions that lead to the Goursat problem. The same methods of analysis however, are extended to the Cauchy problem and the initialboundary value problem. Stability and convergence are defined for a class of finite difference problems and an equivalence theorem is derived which relates the two concepts. Sufficient conditions for stability and convergence are obtained for a particular explicit difference scheme. By a method similar to that used by Fritz John for parabolic equations (Comm. on Pure and Appl. Math. vol. 5 (1952) pp. 155-211), existence and uniqueness theorems are derived. This provides a systematic method for bounding the truncation error. Conditions for stability and convergence for quasi-linear systems are also given. (Received April 15, 1957.)

752. W. K. Ergen and J. A. Nohel (p): Stability of the continuous medium reactor.

Criteria have been obtained recently for the stability of various types homogeneous and heterogeneous reactors by giving sufficient conditions for the stability of equilibrium solutions of the system of nonlinear ordinary differential equations which describe, under suitable restrictions, the dynamics of the reactor (W. K. Ergen, H. J. Lipkin, J. A. Nohel, Applications of Liapounov's second method in reactor dynamics, to appear, J. M. P., 57). In the continuous medium reactor-a limiting case of the heterogeneous reactor-the dynamic equations consist of a nonlinear differentialintegral equation and a diffusion type partial differential equation; here the methods developed previously cannot be applied directly. However, a generalization of these together with Welton's Criterion for stability (see W. K. Ergen, Kinetics of the circulating-fuel nuclear reactor, Journal of Applied Physics, June, 1954), does yield sufficient conditions for stability in this case. Unfortunately Welton's Criterion hinges on an assumption which though physically reasonable is yet to be rigorously justified. A proof of Welton's Criterion using this assumption is also included. (Received June 10, 1957.)

753. Abolghassem Ghaffari: On some mathematical properties of wedge solutions.

The notation and references are the same as in Bull. Amer. Math. Soc. Abstract 63-4-467. It was found that the solution for the stream function $\psi$ of a steady, irrota- 
tional, subsonic compressible flow past a wedge is either: (I) for $m=(n+1) / a$ and $\tau<\tau_{1}, \quad \psi=l g_{1} \sum_{n=0}^{\infty}\left(\psi_{m}(\tau) / \psi_{m}\left(\tau_{1}\right)\right)$ sin $((n+1) / a) \theta$ or (II) for $m=n / a$ and $\tau>\tau_{1}$, $\psi=l g_{1} \sum_{n=0}^{\infty}\left(\psi_{-m}(\tau) / \psi_{-m}\left(\tau_{1}\right)\right) \sin (n / a) \theta$, where $\psi_{m}(\tau)=\tau^{m / 2} F\left(a_{m}, b_{m}, c_{m} ; \tau\right), \psi_{-m}(\tau)$ $=\tau^{-m / 2} F\left(a_{m}+1-c_{m}, b_{m}+1-c_{m}, 2-c_{m} ; \tau\right)$ and $0 \leqq \tau \leqq 1 / 6$. It is proved that in the subsonic interval, $0 \leqq \tau \leqq 1 / 6, \psi_{m}(\tau)$ is a positive monotonically increasing function of $\tau$, and $\psi_{-m}(\tau)$ is a positive decreasing function of $\tau$. When $\tau$ exceeds $1 / 6, \psi_{m}(\tau)$ is an oscillatory function of $\tau$ and its period of oscillation decreases as $\tau$ increases. For large $m$, Shen Yun (F.M. 973-9873) obtained an asymptotic expansion of $\psi_{m}(\tau)$ and also showed that $\psi_{-m}(\tau)$ is numerically smaller than its value at $\tau=1 / 6$. One can show that in the supersonic interval, $1 / 6 \leqq \tau \leqq 1$, only the solution (II) is valid. (Received July 10,1957.)

754. Seymour Ginsburg: On the length of the smallest uniform experiment which distinguishes the terminal states of a machine.

The terminology used is the same as in Gedanken-experiments on sequential machines, by E. F. Moore, appearing in the Annals of Mathematical Studies, No. 34; Automata studies. The problem considered here is to obtain estimates of the length of the smallest experiment (on a machine) which is independent of the unknown initial state and which allows us, by observing the outputs, to distinguish the terminal state. The estimates obtained depend, of course, on the assumptions placed on the machine. In general, the bounds derived are less than $n k, n$ being the number of distinguishable states in the machine and $k$, the number of admissible initial states. For no really general class of machines is a best bound known. (Received June 13, 1957.)

755t. Peter Henrici: Symmetric Runge-Kutta methods for integrating ordinary differential equations of the first order.

The ordinary Runge-Kutta methods for integrating $y^{\prime}=f(x, y)$ are of the form $y_{n+1}-y_{n}=h \Phi_{n}$, where $\Phi_{n}$ is a combination of explicitly calculable values of $f(x, y)$ which differs from $y_{n}^{\prime}+(1 / 2 !) h y_{n}^{\prime \prime}+\cdots$ by $O\left(h^{k}\right), k$ being the order of the method. The paper exhibits "symmetric" Runge-Kutta methods of the form $y_{n+1}-y_{n-1}=2 h \psi_{n}$, where $\psi_{n}$ approximates $y_{n}^{\prime}+(1 / 3 !) h^{2} y_{n}^{(3)}+\cdots$. For $k=2$ there results the midpoint rule, $\psi_{n}=f\left(x_{n}, y_{n}\right)$. All possible forms of $\psi_{n}$ requiring four substitutions are given for $k=4$, and a two-parameter family of expressions $\psi_{n}$, requiring 12 substitutions, is constructed for $k=6$. Symmetric Runge-Kutta methods are only conditionally stable and are not self-starting, but there is the following advantage: If the starting values are judiciously chosen, the overall error ( $x$ fixed, $h \rightarrow 0$ ) satisfies $\epsilon(x)=\eta(x) h^{k}$ $+O\left(h^{k+2}\right), \eta(x)$ being the solution of a well-defined differential equation. Hence Richardson's deferred approach to the limit improves the accuracy by two orders of magnitude. Extensions to systems of equations are also given. (Sponsored by the Office of Ordnance Research.) (Received July 5, 1957.)

756t. Peter Henrici: On the error of a method of Hammer and Hollingsworth for integrating ordinary differential equations.

P. C. Hammer and J. W. Hollingsworth (MTAC vol. 9 (1955) pp. 92-96) proposed a step-by-step method for integrating $y^{\prime}=f(x, y)$ which is based on the two-point (fourth order) Gaussian quadrature formula. The error of the method after one step was implied to be (1) $h^{5} y^{(5)} \xi / 4320$. It is now shown that the overall error ( $x$ fixed, $h \rightarrow 0$ ) is of the form (2) $\epsilon(x)=h^{4} \eta(x)+O\left(h^{5}\right)$. Here $\eta(x)$ is the initially vanishing solution of (3) $\eta^{\prime}(x)=g(x) \eta(x)+\phi(x)$, where $g(x)=f_{y}(x, y(x))$ and $\phi(x)$ is defined in 
terms of derivatives of $f(x, y(x)), y(x)$ being the true solution. For $y^{\prime}=y, y(0)=1$ there results the overall error $\epsilon(x)=-(1 / 720) x e^{x} h^{4}+O\left(h^{5}\right)$, that is, about six times the error that would be present if (1) were correct. [For the ordinary fourth-order Runge-Kutta method the error is $-(1 / 120) x e^{x} h^{4}+O\left(h^{5}\right)$; for Simpson's rule, if properly started, $+(1 / 180) x e^{x} h^{4}+O\left(h^{6}\right)$.] The full accuracy of the Gaussian formula is not realized because the values of $y(x)$ at the irrational abscissas are predicted with insufficient accuracy. Analogous results are proved for the application of the Hammer-Hollingsworth method to systems of equations. (Sponsored by the Office of Ordnance Research.) (Received July 5, 1957.)

757t. Peter Henrici: Methods for integrating ordinary differential equations based on Gaussian quadrature.

If a Gaussian quadrature formula involving $N$ abscissas is used for evaluating $\int_{x_{n}}^{x_{n}+1} f(x, y(x)) d x$ in a step-by-step procedure for solving $y^{\prime}=f(x, y)$, and if the high accuracy of the formula is not to be spoiled, the values of $y(x)$ at the abscissas of integration must be predicted with an error of at most $O\left(h^{2 N+1}\right)$. For the one-point Gaussian formula (midpoint rule) a simplified Runge-Kutta formula can be used as a predictor. There results the formula $y_{n+1}-y_{n}=h f\left(x_{n}+h / 2, y_{n}+(h / 2) f\left(x_{n}+h / 4\right.\right.$, $\left.\left.y_{n}+(h / 4) f\left(x_{n}, y_{n}\right)\right)\right)$. This formula is explicit, self-starting, and strongly stable; it requires 3 substitutions per step. The overall error is $\epsilon(x)=h^{2} \eta(x)+O\left(h^{3}\right)$, where $\eta(x)$ is given by (3) of preceding abstract with $\phi(x)=-(1 / 24) y^{(3)}(x)$ [as contrasted to $-(1 / 12) y^{(3)}$ for the trapezoidal rule and $-(1 / 6) y^{(3)}$ for $\left.y_{n 1}-y_{n-1}=2 h f_{n}\right]$. Similar results are obtained for the two-point Gaussian rule used with the ordinary fourth order Runge-Kutta formula as a predictor. The error then satisfies (2) and (3) of preceding abstract with $\phi(x)=-(1 / 4320) y^{(5)}(x)$, at the expense of 9 substitutions per step. Example: For $y^{\prime}=y, y(0)=1$ the error is $\epsilon(x)=-(1 / 4320) x e^{x} h^{4}+O\left(h^{5}\right)$. Methods and results concerning error generalize to systems of equations. (Sponsored by the Office of Ordnance Research.) (Received July 5, 1957.)

\section{8t. Erwin Kreyszig: On relaxation oscillations.}

The van der Pol equation can be generalized in different ways. Of particular interest are those generalized equations whose solutions can be investigated by special methods which yield more detailed knowledge than the general theory of relaxation oscillation does. The equation (1) $\ddot{q}-(\operatorname{sgn} \dot{q}) \frac{1}{2}\left(a-b \dot{q}^{2}\right)+2^{-1} c f(q)=0$ is of this type; here $q(t)$ is the elongation, $a, b$, and $c$ are positive constants. (1) can be transformed into (2) $d V / d q-(\operatorname{sgn} \dot{q})(a-b V)+c f(q)=0$ where $V=\dot{q}^{2}$. Let $f(q)$ be non-negative for positive values of $q$ and nonpositive for negative values of $q$. Then the oscillations tend to a limit cycle. If $f(q)=\sum_{s=0}^{n} c_{s} q^{s}$, the solution $V(q)$ of $(2)$ can be represented in terms of elementary functions and the sequence of maximum amplitudes of the oscillation can be determined. In particular, if $f(q)=q^{2 m+1}, m=0,1, \cdots$, the maximum amplitude of the limit cycle is the root of the equation $\left(\sum_{s=0}^{m}((2 s+1) !)^{-1} x^{2 s+1}\right)$ $\cdot\left(k_{m}+\sum_{s=1}^{m}((2 s) !)^{-1} x^{2 s}\right)^{-1}=\tanh x$, where $x=b q$ and $k_{m}$ is a constant, depending on $a$, $b, c$, and $m$. If $f(q)$ satisfies the above condition and is not an odd function, then the positive and negative maximum amplitude of the limit cycle are different from each other. (Received March 28, 1957.)

759t. H. W. Kuhn: A matrix game equivalent to the Transportation Problem.

The Hitchcock-Koopmans Transportation Problem asks for the minimum of the linear form $\sum c_{i j} x_{i j}$ subject to $x_{i j} \geqq 0, \sum_{j} x_{i j}=a_{i}, \sum_{i} x_{i j}=b_{j}$, where $i=1, \cdots, m$, 
$j=1, \cdots, n$ and we may assume $a_{i}>0, b_{j}>0$, and $\sum_{i} a_{i}=\sum_{i} b_{j}$. The following matrix game $\Gamma$ is based on the same data: Player A chooses an index $i$ from $1, \cdots, m$. Independently, Player $\mathrm{B}$ chooses, for each $j=1, \cdots, n$, an index $k(j)$ from $1, \cdots, m$. Player B pays Player A the amount $C a_{i}+\sum_{j} b_{j}\left(c_{k(j), j}-\left(C \delta_{k(j), i}\right)\right.$ where $C$ is a constant greater than $(m-1) \max _{j} \max _{k l},\left(c_{k j}-c_{l j}\right), \delta_{k i}=1$ if $k=i$, and $\delta_{k i}=0$ if $k \neq i$. Theorem: The matrix game $\Gamma$ is equivalent to the Transportation Problem. The $n$ distributions $q_{i j} \geqq 0, \sum_{i} q_{i j}=1$ constitute an optimal (behavior) strategy for $B$ if and only if $x_{i j}=b_{j} q_{i j}$ solve the Transportation Problem. The distribution $p_{i} \geqq 0, \sum_{i} p_{i}=1$ is an optimal (mixed) strategy for $A$ if and only if $u_{i}=C p_{i}$ and $v_{j}=\min _{k}\left(c_{k j}-u_{k}\right)$ solve a program dual to the Transportation Problem, namely: Maximize $\sum_{i} a_{i} u_{i}$ $+\sum_{j} b_{j} v_{j}$ subject to $u_{i}+v_{j} \leqq c_{i j}, u_{i} \geqq 0$, and $\sum_{i} u_{i}=C$. Preliminary computational experience using fictitious play favors this game over other matrix game versions of the problem. (Received July 16, 1957.)

760. H. M. Lieberstein: On a mixed problem for the Euler-PoissonDarboux equation.

Solution of the following boundary value problem is given here. Find a regular solution $u^{(k)},-\infty<k<1$, of the two dimensional EPD equation $u_{x x}^{(k)}=u_{y y}^{(k)}+(k / y) u_{y}^{(k)}$, such that $u^{(k)}(x, 0)=f(x)$ and $u^{(k)}(x, x)=g(x)$. Here $f(x)$ and $g(x)$ are defined on $[0, a],[0, b]$, respectively, have a specified number of continuous derivatives there and another specified number of vanishing derivatives at the origin, both numbers depending on $k$. This problem was solved earlier for $g=0$ and for somewhat more restrictive conditions on $f$ by A. Weinstein [Summa Brasiliensis Mathematicae vol. 3, September, 1955]. Uniqueness considerations given by the author in another paper cover this problem. The general solutions of E. K. Blum [Duke Math. J. vol. 21, pp. 257-274] and certain Riemann-Liouville integrals are used to obtain solutions for $-2<k<1$, and solutions for all negative $k$ are generated using the recursion, $u^{(k)}=(y /(k+1)) u_{y}^{(k+2)}+u^{(k+2)}$, due to L. Payne. It is found that polynomial data yield polynomial solutions if and only if $k$ is a negative even integer though poly. nomial solutions do exist for other values of $k$. A generalized Huyghens' principle is noted for $k=0$ only. (Received February 18, 1957.)

\section{Walter Littman: On Struik's gravity waves.}

Struik's proof for the existence of two dimensional periodic gravity waves in a canal of finite depth (Math. Ann. vol. 95 (1926) pp. 595-634) does not offer a convenient means of studying its own range of validity in terms of the physical variables involved. In particular, it is not clear that the interval of convergence of the series expansion (on which the proof is based) remains bounded away from zero as $h \rightarrow \infty$ ( $h=$ depth) thus making it impossible to obtain the case of infinite depth (treated earlier by Levi Civita) in the limit as $h \rightarrow \infty$. Replacing the power series expansion of Struik's proof by an iteration scheme (cf. Stoker, Water waves, Interscience Publishers, to appear, section 12.2) results in a somewhat simplified proof whose range of validity can be studied more easily, in terms of the physical variables. In particular the case of infinite depth is obtained in the limit as $h \rightarrow \infty$. Approximate formulas for various physical quantities are derived. This proof also extends to flows over certain periodic bottoms which are "approximately horizontal." (Received July 12,1957.)

762. Mark Lotkin: Integration of heat conduction equations for composite media.

The quasi-linear parabolic differential equation $c^{(m)} \rho^{(m)}(\partial u / \partial t)=\partial / \partial x\left(k^{(m)} \partial u / \partial x\right)$, 
$m=1,2, \cdots$, together with the accessory boundary, initial, and interface conditions governing the conduction of heat through composite media are approximated by a tri-diagonal system of finite difference equations. By considering the propagation of the local truncation error the recurrence relations for this error are established. Assuming the boundedness in the basic region of definition of certain derivatives of the heat function $u$ up to the fourth order, the boundedness of the overall truncation error is assured, thus proving the convergence of the solution of the difference equation to that of the differential equation. The treatment is in some respects similar to that discussed by Douglas, Pacific J. Math. vol. 6 (1956). (Received May 13, 1957.)

\section{Walter Noll: Relativistic aspects in classical mechanics.}

Classical mechanics must nowadays be regarded as essentially identical with the mechanics of continuous media. A satisfactory axiomatic foundation of continuum mechanics has never been given, and an attempt in this direction is made here. Precise definitions in terms of vector measures of such notions as force, mutual force, contact force, body force, and external force are given. It turns out that a basic axiom is a principle of relativity, which states that the mutual action between bodies is independent of the frame of reference. The particular role of Galilean frames of reference disappears in an appropriate formulation of the theory. The principle of relativity has far reaching consequences in the form of invariance requirements for general constitutive equations, i.e. equations describing the mechanical properties of general classes of ideal materials. These general materials have been the object of much research recently. (Received July 5, 1957.)

\section{R. W. Preisendorfer: Radiative transfer and measure theory.}

It is shown that four structure axioms $\mathrm{C}_{1}, \mathrm{M}_{1}, \mathrm{R}_{1}, \mathrm{~T}_{1}$, formulated in terms of modern measure theory can serve as a mathematical foundation for radiative transfer theory. $\mathrm{C}_{1}$ defines a carrier space: a measure space with totally sigma-finite measure on a sigma-algebra: $(X, S, \nu) . M_{1}$ deals with a non-negative cone $\Re \eta_{\nu}$ of $\nu$-continuous measures on $S$ (the radiative measures). $R_{1}$ and $T_{1}$ employ abstract variants of the major premise of Huygens' Principle: $R_{1}$ defines a family $R_{\phi}$ (the radiative process) of linear transformations $R_{t, t^{\prime}}$ on $\mathfrak{T}_{\nu}$ into $\mathfrak{T}_{\nu} ; \mathrm{T}_{1}$ defines a family $\mathfrak{J}_{\psi}$ (the transfer process) of measure preserving transformations $T_{t, t^{\prime}}$ on $(X, S, \nu)$ onto $(X, S, \nu)$. The pair $\left(\cap_{\phi}, J_{\psi}\right)$ is the radiative transfer process. Polarization is included in the formulations. Results: solution of the long-standing problem of Hopf; connections between r.t. and electromagnetic theory via generalized harmonic analysis; connections between r.t. and Mueller phenomenological optics algebra; subsumption of classical r.t. and neutron transport theories. Reference: R. Preisendorfer, $A$ mathematical foundation for radiative transfer theory. Dissertation, University of California at Los Angeles, May, 1956. (Received July 2, 1957.)

\section{5t. I. F. Ritter: The optimum linear program.}

The theory of the Linear Programming problem-to maximize a linear form of many real variables constrained by linear inequalities-together with its dual minimum problem, is derived by most elementary algebra. On this is based a practical method of computing simultaneously the solutions of the dual problems. Running parallel with the well-known Simplex Method, the present algorithm employs a rectangular array that differs essentially from the Simplex Tableau, particularly by 
the explicit appearance of the edge vectors leading away from any vertex of the polyhedron of admissible vectors. Besides some simplification of the computational routine, advantages derived from using the new array are: The difficulties of the Simplex Method in securing an initial "basic feasible solution" and in dealing with "degeneracy" are avoided. A correction method is readily available for the reduction of rounding errors. If the problem is not too large, it can be solved under the additional constraint that the solution must consist of integers. (Received July 1,1957.)

\section{6t. H. E. Salzer: Formulas for hyperosculatory interpolation, direct and inverse.}

A convenient new procedure for direct "hyperosculatory" interpolation for $f(x)=f\left(x_{0}+p h\right) \equiv f$, when given $f\left(x_{i}\right) \equiv f_{i}, f^{\prime}\left(x_{i}\right) \equiv f_{i}^{\prime}$ and $f^{\prime \prime}\left(x_{i}\right) \equiv f_{i}^{\prime \prime}$ at $n$ equally spaced points $x_{i}=x_{0}+i h, i=-[(n-1) / 2]$ to $[n / 2]$, is derived from Hermite's $(3 n-1)$ th degree osculatory interpolation formula, for $n=2(1) 7$, (i.e. up to 20th degree accuracy). Certain fixed auxiliary quantities $a_{i}, b_{i}$ and $c_{i}$, which are independent of $p, f_{i}, f_{i}^{\prime}$ and $f_{i}^{\prime \prime}$, are tabulated exactly. The method is an extension of the author's earlier adaptation of the simple osculatory interpolation formula, employing both a decomposition and uniqueness property of Hermite's general formula. The remainder term indicates the vast increase in accuracy and permissible size of $h$, when compared with simple osculatory interpolation formulas which are the next most accurate. For inverse interpolation the coefficients of the first ten powers of $r=\left(f-f_{0}\right) / h f_{0}^{\prime}$, for $n=2(1) 5$, (i.e. up to 14 th degree accuracy in the direct function) are given in terms of $f_{i}, f_{i}^{\prime}$ and $f_{i}^{\prime \prime}$. Hyperosculatory interpolation is specially suitable in (1) practical problems in astronautics involving rocket or missile flight, where the acceleration data, or $f_{i}^{\prime \prime}$ are available as well as position and velocity data, or $f_{i}$ and $f_{i}^{\prime}$, and where (2) higher mathematical functions that are tabulated with their first derivatives, are solutions of simple second order differential equations, so that $f_{i}^{\prime \prime}$ is readily obtained. (Received June 20, 1957.)

767t. H. E. Salzer: Toward a Gibbsian approach to the problems of growth and cancer, I: Growth potentials.

Certain sections of Josiah Willard Gibbs' thermodynamics papers might be applicable to biological equilibrium and growth, normal or abnormal. Gibbs added terms $\sum \mu_{i} d m_{i}$ to the differential of the internal energy $d \epsilon=t d \eta-p d v$, ( $t=$ temperature, $p=$ pressure, $\eta=$ entropy, $v=$ volume) where $\mu_{i}=\partial \epsilon / \partial m_{i}$ is the potential of substance $m_{i}$, to provide for chemical as well as thermal and mechanical equilibrium. In this article a further generalization is suggested, to include biological equilibrium by adding to $d \epsilon$ terms of the form $G d N$, the variable $N$ being the number of cells, where $G=\partial \epsilon / \partial N$ is a "growth potential" that measures exactly the resistance toward spontaneous growth. The function $G$, like $\mu_{i}$, is intensive in nature (i.e. depends on intensive variables only) except for a conversion factor $d M / d N, M=\sum m_{i}$, affording possible insight into why incipient abnormal growth is often independent of the number of cells. Useful applications might follow from identities between $\partial G / \partial \eta$, $\partial G / \partial v$ or $\partial G / \partial m_{i}$ and $\partial t / \partial N,-\partial p / \partial N$ or $\partial \mu_{i} / \partial N$ respectively. The following new function is studied, $\bar{\zeta}=\zeta-G N$, a natural generalization of the Gibbs free energy function $\zeta$, the possibility of measuring it electrically, and comparison of its role with that of $\zeta$ for the possible experimental determination of $G$. (Received July 15, 1957.) 
768t. H. E. Salzer: Toward a Gibbsian approach to the problems of growth and cancer, II : Equilibrium, stability, pressure, surface tension.

Gibbs' necessary and sufficient conditions for heterogeneous equilibrium of $n$ components in $m$ phases are generalized and also modified to include broader restraining conditions like $\sum_{i=1}^{m} \delta N_{j}^{(i)} \geqq 0, j=1, \cdots, n$, the $>$ being characteristic of only living cellular phases. Careful appraisal of the term "biological stability" is followed by new criteria for stability, instability, and limits of stability (neutral equilibrium) in terms of derivatives of $G$, with possible medical applications. Three different sections of Gibbs' works tend to indicate that, for a biological phase, lower pressure usually increases its stability. The equation $p^{\prime \prime}-p^{\prime}=\sigma\left(1 / r+1 / r^{\prime}\right)$, where $\sigma=$ surface tension, $p^{\prime \prime}, p^{\prime}=$ pressures, $r, r^{\prime}=$ radii of curvature, is applied to possible control of tissue growth at interfaces. Methods of altering the equilibrium between three phases $A, B, C$ by varying the interfacial tensions $\sigma_{A B}, \sigma_{B C}, \sigma_{A C}$, using relations like $\sigma_{A B}<\sigma_{A C}$ $+\sigma_{B C}$ for stability of the $A, B$ interface, suggest different means for shifting biological equilibrium between normal and abnormal cells through the introduction of new third phases at the interface. Various devices are mentioned for possible control of growth through proper channeling of surface or other equivalent forms of energy. (Received July $15,1957$.

769. D. E. Spencer: Exact and approximate solutions of integral equations.

Approximate solutions of the Fredholm-type integral equations of interflection theory have previously been obtained (P. Moon and D. E. Spencer, J. Franklin Inst., vol. 242 (1946) p. 111; vol. 250 (1950) p. 151. Exponential approximations to the kernels were employed and exact solutions of the resulting approximate integral equations were obtained. A new method permits an exact solution to be obtained, by solving an auxiliary integral equation. The method is applied to the infinitely long cylindrical black body and to finite cylinders of various cross sections. A theoretical basis is thus provided for determining the accuracy of the earlier approximate solutions. (Received July 15, 1957.)

\section{0t. R. L. Sternberg, J. S. Shipman, and L. S. Rose: Multiple Fourier analysis in rectifier problems.}

The nonlinear problem of the multiple Fourier analysis of the output from a cutoff power law rectifier responding to a several-frequency input is reviewed for the one- and two-frequency problems and is briefly investigated for the three-frequency problem. The solutions for the modulation product amplitudes or multiple Fourier coefficients are obtained in exact although transcendental form. An account of the mathematical properties of these multiple Fourier coefficients or Bennett functions, including hypergeometric representations and power series expansions for them as well as recurrence relations satisfied by them, is given in the paper together with numerical tables of the first ten basic functions for the one-frequency problem and of the first fifteen basic functions for the two-frequency problem. Further applications of the theory are also given to the computation of average output power with the aid of the multiple Fourier coefficients or Bennett functions tabulated in the paper, and the work is concluded with some brief remarks concerning the interpretation of the results in terms of the theory of almost periodic functions and the generalized Fourier series of Bohr under appropriate conditions. The entire theory is based on the original 
method of the expansion of the rectifier output in multiple Fourier series introduced by Bennett in 1933 and 1947. (Received June 14, 1957.)

771. R. S. Varga: The p-color problem: A generalization of the Young-Frankel successive overrelaxation scheme.

The Young-Frankel method of successive overrelaxation, which has been shown by Young [Trans. Amer. Math. Soc. vol. 76, pp. 92-111] to correspond to a two color problem, is generalized to a method of successive overrelaxation for a $p$ color problem, $p \geqq 2$, the results holding for either point or line relaxation. The generalization, based on works of Romanovsky and Frobenius concerning nonnegative matrices, makes use of the properties of cyclic matrices of index $p$. The fundamental equation relating eigenvalues of iteration matrices becomes $(\lambda+\omega-1)^{p}$ $=\lambda^{p-1} \omega^{p} \mu^{p}$. An analogous formula for the optimum overrelaxation factor $\omega$ is derived, and an application is given to the solution of elliptic difference equations on a triangular mesh. (Received June 10, 1957.)

\section{Paul Weiss: On the evaluation of the general bialternant.}

Consider $n$ functions $f_{1}(x), \cdots, f_{n}(x)$ of one independent variable $x$. Let $x$ take on $n$ distinct values $x_{1}, \cdots, x_{n}$. Form the general alternant of the $f_{i}\left(x_{k}\right)$, i.e. the determinant $\left|f_{i}\left(x_{k}\right)\right|$. Divide this determinant by the simple alternant $\left|x_{k}^{(i-1)}\right|$. The resulting bialternant can be expressed as a $(n \times n)$ determinant whose element in the $i$ th row and $k$ th column is the $(n-1)$ st divided difference of the function $x^{n-k} f_{i}(x)$. This result reduces (1) to Jacobi's classical bialternant theorem if the functions $f_{i}(x)$ are monomials $x^{r_{i}},(2)$ to the expression of the $(n-1)$ st divided difference as a quotient of two $(n \times n)$ determinants (see e.g. W. E. Milne: Numerical calculus, Princeton 1949, p. 206) if $f_{i}(x)=x^{(i-1)}, i=1,2, \cdots, n-1 ; f_{n}(x)=F(x) ;(3)$ by a limiting operation it leads to a general mean-value theorem due to Schwarz and Stieltjes (T. J. Stieltjes: Oeuvres, vol. 2, p. 110; G. Pólya und G. Szegö: Aufgaben und Lehrsatze II, ex. V, 95, p. 54). (Received July 11, 1957.)

\section{GEOMETRY}

773. D. C. Benson and L. E. Dubins (p): On subsets of the unit sphere, which are sufficiently small to be subsets of some hemisphere.

The authors show that if an arcwise connected subset of the surface of the unit sphere, $S^{2}$, has a diameter less than $2 \pi / 3$, then it is a subset of some open hemisphere of $S^{2}$. Furthermore $2 \pi / 3$ is best possible. (By the diameter of a subset $A$ of $S^{2}$ is meant, as usual, the least upper bound of the distances between pairs of points of $A$ where the distance is the length of the smaller great circular arc joining the pair of points.) (Received July 12, 1957.)

\section{4t. D. W. Crowe: On the geometry of quaternions.}

It is known that the plane $\pi(A)$ in real euclidean 4 -space $E^{4}$ defined by the complex equation $w=A z,(w=u+i v, A=a+i b, z=x+i y)$ (i.e. by the two real equations $u=a x-b y, v=b x+a y)$ is isocline to the plane $\pi(0)$ at the origin. We consider the analogous properties of the 4-dimensional subspace $P(R)$ of $E^{8}$ defined by the quaternion equation $p=R q, p, R, q$ quaternions (i.e. by four real equations). It is shown that $P(R)$ is isocline to $P(0)$ at the origin and that the angle $\alpha$ between two such 4-spaces $P(R), P(S)$ is given by $\cos ^{2} \alpha=\left|1+R S^{*}\right|^{2} /\left(1+R R^{*}\right)\left(1+S S^{*}\right)$ where $R^{*}$, 
$S^{*}$ denote the quaternion conjugates of $R, S$. This is the same formula as that for the angle between two planes in $E^{4}$ (with "quaternion conjugate" replaced by "complex conjugate"). This implies that the sphere model and stereographic projection property of isocline planes in $E^{4}$ (Bull. Amer. Math. Soc. Abstract 63-1-53) can be extended to the case of isocline 4-spaces in $E^{8}$. Similar results hold for 4-spaces defined by $p=R q^{*}$. (Received July 1,1957 .)

\section{Michael Goldberg: Intermittent rotors.}

Rotors include noncircular shapes which may be rotated through all orientations in the plane while keeping contact with a number of fixed elements such as points, lines or circles. Since only three contacts are sufficient to constrain the motion, the rotors may be modified to remove some of the excess constraints. The rotor then makes contact, not continuously with all the fixed elements, but intermittently with different sets of the elements. Intermittent rotors derived in other ways are also shown. Of particular interest is the shape of least area which, when placed at random on a square lattice of points, always includes at least one of the points. This was shown by J. J. Schäffer [Math. Ann. vol. 129 (1955) pp. 265-273] and D. B. Sawyer [Quart. J. Math. vol. 4 (1953) pp. 284-292] to be a square to which has been added the areas enclosed by two parabolic arcs. This shape is an intermittent rotor which may be rotated while passing through at least three vertices of a fixed square. (Received July 3, 1957.)

\section{V. G. Grove: Closed convex surfaces.}

Let $S$ be a closed, convex, orientable surface of class $C^{\prime \prime \prime}$. Let the second fundamental form of $S$ be chosen as positive definite. Let $F$ be a positive definite form on $S$ whose coefficients $b_{\alpha \beta}$ are such that $b_{\alpha \beta, \gamma}-b_{\alpha \gamma, \beta}=0$. Such a form will be called trisymmetric on $S$. Let the discriminants of $F$ and of the first and second fundamental forms be $b, g$ and $d$ respectively, and let $J=b / g, K=d / g$. Let $\bar{S}$ be a second closed, convex orientable surface of class $C^{\prime \prime \prime}$, whose second fundamental form is positive definite. Let $h$ be a differentiable homeomorphism of $S$ into $\bar{S}$. Let $\bar{F}$ be the image of $F$ under $h, \bar{F}$ being assumed trisymmetric on $\bar{S}$. Then if $\bar{J}=J, \bar{K}=K$ at corresponding points under $h$, and if $h$ is orientation preserving then $h$ is a rigid motion. (Received July 1, 1957.)

\section{David Hertzig: On simple algebraic groups. Preliminary re-} port.

Let $G$ be a connected linear algebraic group defined over a field $k$; an algebraic group $G^{\prime}$ defined over $k$ is a $k$-form of $G$ split by $K$ if there is an isomorphism between $G$ and $G^{\prime}$ defined over an algebraic extension $K$ of $k$. When $K$ is a Galois extension of $k$ with Galois group $\Gamma$ the distinct $k$-forms of $G$ split by $K$ are in one-to-one correspondence with the 1-dimensional cohomology set $H^{1}\left(\Gamma\right.$, Aut $\left._{K} G\right)$ where Aut $_{K} G$ is the group of automorphisms of $G$ defined over $K$ (particular case of a theorem of Weil). The following is derived from a theorem of Chevalley: Let $G^{\prime}$ be a simple linear algebraic group defined over $k$. Then there exists a group $G$ defined over the prime field such that $G^{\prime}$ is a $k$-form of $G$; $G$ is separably isogenous to a pseudo-classical or pseudo-exceptional group. The pseudo-classical and pseudo-exceptional groups are certain groups described by Dieudonne which are inseparably isogenous to classical and exceptional groups. Their Lie algebras are constructed, following Chevalley; the $k$-forms are then obtained, with a few exceptions. Due to the principle of triality new simple Lie algebras, hence new simple algebraic groups, are constructed as forms of the classical Lie algebra of type $D_{4}$. (Received July $15,1957$. ) 
778. C. C. Hsiung: Curvature and Betti numbers of compact Riemannian manifolds with boundary.

The purpose of this paper is to study harmonic and Killing tensor fields on compact orientable Riemannian manifolds $M^{n}$ of dimension $n(\geqq 2)$ and class $C^{3}$ with connected boundary $B^{n-1}$ of dimension $n-1$. By using a duality theorem of Lefschetz and a theorem of Duff and Spencer (Ann. of Math. vol. 56 (1952), pp. 128-156), a typical result can be stated as follows: On a $M^{n}$ not homeomorphic to an n-dimensional submanifold, with an (n-1)-dimensional boundary, of an n-dimensional Euclidean space, if the Ricci curvature is positive definite everywhere; then there exists no harmonic vector field, other than the zero vector field, with zero tangential or normal component on $B^{n-1}$, and the absolute first Betti number of $M^{n}$ and the relative first Betti number of $M^{n}$ $\bmod B^{n-1}$ are zero. For the case of empty $B^{n-1}$, this result was proved first by S.B. Myers (Duke Math. J. vol. 8 (1941) pp. 401-404) by calculus of variations, and again by S. Bochner (Bull. Amer. Math. Soc. vol. 52 (1946) pp. 776-797) by an entirely different method with the use of a result of $E$. Hopf on linear elliptic differential equations of the second order. In this paper, Bochner's method is modified by replacing the use of Hopf's result by constructing some differential forms and applying Stokes' theorem. (Received June 18, 1957.)

779. W. R. Hutcherson: Oscillating inequality, Fibonacci sequence, and quadratic transformations.

This paper gives a general proof of an inequality [J. C. Morelock, N. C. Perry, and W. R. Hutcherson, Fibonacci sequence applied to quadratic transformations, Bull. Amer. Math. Soc. Abstract 62-6-596] which oscillates in sense, as one uses the Fibonacci sequence related to quadratic transformations. (May 23, 1957.) (Received May 27, 1957.)

780. T. K. Pan: Existence of surfaces in a certain conformal correspondence.

Let $v$ and $\bar{v}$ be corresponding unit vector fields associated with two conformal surfaces $S$ and $\bar{S}$, respectively. Let the indicatric torsions of $v$ and $\bar{v}$ along two corresponding curves $C$ and $\bar{C}$ be equal (T. K. Pan, Torsion of a vector field, Proc. Amer. Math. Soc. vol. 7 (1956) p. 453). The existence of such surfaces is investigated. Theory of exterior differential system is applied for the study. It is found that the general solution depends on one arbitrary function of two variables. Singular solution, characteristic and Cauchy problem are discussed. (Received July 11, 1957.)

\section{LOGIC AND FOUNDATIONS}

781t. J. W. Addison: Separation principles in the hierarchies of classical and effective descriptive set theory.

Let $\Re$ be the product of $n$ copies of the set $N$ of natural numbers with the discrete topology and $f$ copies of $N^{N}$ under the product topology, where $n, f \in N$ and $n+f>0$. If $C \subseteq N^{N}$ the $C$-arithmetical ( $C$-analytical) hierarchies on $\mathscr{N}$ are those obtained by quantifying over $N$ (over $N^{N}$ ) predicates on $\Re$ recursive in functions of $C$. Thus in particular the $\phi$-arithmetical ( $\phi$-analytical) hierarchy is the arithmetical (analytic) hierarchy of recursive function theory (cf. Kleene, Bull. Amer. Math. Soc. vol. 61 (1955) pp. 193-213) and the $N^{N}$-arithmetical ( $N^{N}$-analytical) hierarchy is the finite Borel (projective) hierarchy of classical descriptive set theory. By a uniform method 
the reduction (1st and 2nd separation) principle(s) is (are) shown to hold (fail) on the $\exists$ and fail (hold) on the $\forall$ side of the $C$-arithmetical and $C$-analytical hierarchies with the exception of the 1st level of the $C$-analytical hierarchies where the situation is exactly reversed, a consequence of the axiom of constructibility being assumed in the proof for the 3rd and higher levels of the $C$-analytical hierarchies. Thus in particular we obtain the result announced without proof by Novikov (cf. Trudy Mat. Inst. Steklova vol. 38 (1951) pp. 279-316) that it is consistent with the axioms of set theory that for some $k$ at the $k$ th and higher levels of the projective hierarchy the separation principles behave as they do at the 2nd level (our result yielding further that $k$ can be chosen as 3). (Received July 3, 1957.)

\section{Ronald Harrop: On the existence of finite models and decision procedures for propositional calculi.}

In this paper certain general properties of propositional calculi are considered. Two forms of the definition of a finite model of a propositional calculus with a finite number of axiom schemes and of rule schemes are discussed, these forms differing in the manner prescribed for the satisfaction of the rule schemes. In the first case it is insisted that when the premises of an application of such a scheme are valid then the conclusion of that application of the scheme must also be valid. In the second case the stronger condition is used that when the premises of a rule scheme take designated values for a certain substitution of values for the variables for arbitrary formulae in the scheme, then, for that substitution of values for the variables, the conclusion also takes a designated value. It is in general much easier to test for satisfaction of the second condition than of the first condition. The methods of definition are shown to be equivalent for the application of the finite model method for the demonstration of the unprovability of formulae in the calculus. It is further proved that, although the decidability of a calculus follows from the existence of a finite model counterexample for each unprovable formula of the calculus, the converse result is not true. (Received June 27, 1957.)

\section{3t. S. C. Kleene: Recursive functionals and quantifiers of finite types. I.}

Natural numbers are of type 0 , functions $\alpha^{j+1}\left(\alpha^{j}\right)$ from type-j objects $\alpha^{j}$ to natural numbers of type $j+1$. The primitive recursive functions $\phi(\mathfrak{A})$, for $\mathfrak{A}$ a list of variables of these types, are those definable by eight schemata S1-S8, e.g.: S8 $\phi\left(\alpha^{j}, \mathfrak{l}\right)$ $=\alpha^{i}\left(\lambda \alpha^{j-2} \chi\left(\alpha^{j}, \alpha^{j-2}, \mathfrak{R}\right)\right)$. They have as indices numbers codifying the schema applications. Partial recursive functions $\phi(\mathfrak{R})$ are those definable by S1-S8 and: S9 $\phi(a, \mathfrak{B}, \mathfrak{E}) \cong\{a\}(\mathfrak{R})$ where $\{a\}(\mathfrak{R})$ is the value if defined of the function with index $a$ for arguments $\mathfrak{R}$ (and S9 is included in the indexing). A completely defined partial recursive function is general recursive. Partial $\mu$, and $\mu$-, recursive functions are obtained when S9 is replaced by: $\mathrm{S} 10 \phi(\mathfrak{H}) \cong \mu y[\chi(\mathfrak{A}, y)=0]$. For $\mathfrak{A}$ of types $\leqq 1$, the notions coincide with the former ones. Otherwise, $\mu$-recursiveness is narrower than general recursiveness. Indeed, let $\mathrm{E}\left(\alpha^{1}\right)=0$ if $(E a)\left[\alpha^{1}(a)=0\right],=1$ otherwise; then for number variables $\mathfrak{R}$, the functions $\lambda \mathfrak{R} \phi(\mathfrak{R}, \mathrm{E})$ for $\phi\left(\mathfrak{R}, \alpha^{2}\right) \mu$ - (general) recursive are exactly the arithmetical (hyperarithmetical) functions. The general recursive functions are closed under substitution of functionals $\lambda \alpha^{j} \theta\left(\mathfrak{A}, \alpha^{j}\right)$ ( $\theta$ general recursive). For $\mathfrak{A}$ of types $\leqq r$, if $\phi$ is partial recursive with index $z, \phi(\mathfrak{R}) \cong w$ is expressible by either $\left(\beta^{r-1}\right)\left(E \xi^{-2}\right)$ or $\left(E \beta^{r-1}\right)\left(\xi^{r-2}\right)$ prefixed to a primitive recursive predicate of $z$, etc., if $r>2$, (but if $r=2$, by only the first). (Received June 13, 1957.) 
784t. Richard Montague: An extension of Gödel's incompleteness theorem.

For terminology see Tarski, Mostowski, Robinson, Undecidable Theories. If $S, T$ are theories without common (nonlogical) constants and there is a one-place predicate belonging to neither, then $A$ is said to be an interpretation of $S$ in $T$ if $A$ is a set of possible definitions in $T$ such that $S^{(\pi)} \subseteq T+A$, for some one-place predicate $\pi$ which belongs to neither $S$ nor $T$, and $A$ contains at most one possible definition for each constant. The extension of this notion to arbitrary theories is obvious. Let $P$ be Peano's arithmetic. Rosser's version of Gödel's theorem may be stated as follows: For any recursively axiomatizable and consistent theory $T$ and any interpretation $A$ of $P$ in $T$, there is a sentence $\phi$ of $P$ such that neither $\vdash{ }_{T+A} \phi$ nor $\vdash_{T+A} \sim \phi$. Extension: For any recursively axiomatizable and consistent theory $T$, there is a sentence $\phi$ of $P$ such that, for any interpretation $A$ of $P$ in $T$, neither $\vdash_{T+A}$ nor $\vdash_{T+A} \sim \phi$; further, $P$ may be replaced here by a certain finitely axiomatizable subtheory of $P$. Thus the choice of an undecidable sentence need not depend on the choice of definitions for the constants of arithmetic. (Received July 12,1957.)

\section{Statistics and Probability}

785. D. G. Austin and R. V. Chacon (p): The continuity postulate for Markov processes-Abstract space case. Preliminary report.

We show that a set of necessary and sufficient conditions for the continuity of the transition probabilities of an abstract space, continuous parameter Markov process (i.e. the transition probabilities $P_{t}(x, A)$ are defined for $t \in(0,+\infty), x \in X$ and $A \in B$ where $B$ is a Borel field of subsets of the abstract space $X$ ) is: (i) for all $x$ and $A P .(x, A)=0$ a.e. $\Rightarrow P .(x, A) \equiv 0$, (ii) for all $x$ and $A P .(\cdot, A)$ is measurable $(0,+\infty) \times X$ and $P .(x, A)$ is measurable $(0,+\infty)$. We then use this result to show that the process is continuous under several modifications of Doob's continuity postulate, for all $x, \lim _{t \rightarrow 0} P_{t}(x,\{x\})=1$. (Received August 5, 1957.)

\section{G. E. Baxter: An operator identity.}

For any matrix $N$ let $N^{+}$be the matrix formed from $N$ by setting equal to zero all elements of $N$ which lie on or below the diagonal, and let $N^{-}=N-N^{+}$. Define sequences $\left\{P_{k}\right\}$ and $\left\{Q_{k}\right\}$ by $P_{0}=Q_{0}=I, P_{n+1}=\left(M P_{n}\right)^{+}$, and $Q_{n+1}=\left(Q_{n} M\right)^{-}$. If $P$ and $Q$ denote the generating functions (in $s$ ) of $\left\{P_{k}\right\}$ and $\left\{Q_{k}\right\}$, then (i) $P=I$ $+s(M P)^{+}$, (ii) $Q=I+s(Q M)^{-}$, (iii) $P Q=(I-s M)^{-1}$, and (iv) if $\left(M^{k}\right)^{+} M=M\left(M^{k}\right)^{+}$ for all $k=1,2, \cdots$, then $P=\exp \left(\sum_{1}^{\infty} s^{k}\left(M^{k}\right)^{+} / k\right)$ and $Q=\exp \left(\sum_{1}^{\infty} s^{k}\left(M^{k}\right)-/ k\right)$. These results have application to probability theory. If $M$ is the transition probability matrix of a Markov chain $\left\{X_{k}, k \geqq 0\right\}$, with states $\alpha_{1}<\cdots<\alpha_{K}$, and if $\left({ }_{n} p_{i j}\right)=P_{n}$ and $\left.{ }_{n} q_{i j}\right)=Q_{n}$, then ${ }_{n} p_{i j}=P\left\{\max \left(X_{0}, \cdots, X_{n-1}\right)<X_{n}, X_{n}=\alpha_{j} \mid X_{0}=\alpha_{i}\right\}$ and ${ }_{n} q_{i, j}=P\left\{\max \left(X_{0}, \cdots, X_{n}\right)=X_{0}, X_{n}=\alpha_{j} \mid X_{0}=\alpha_{i}\right\}$. If (iv) holds, the $P$ and $Q$ form an operator analogue of Spitzer's identity (Trans. Amer. Math. Soc. vol. 82 (1956) pp. 323-339). These results have been extended to hold for certain integral operators of the form $M f=\int_{-\infty}^{\infty} f(y) m(x ; d y)$. (Received July 8,1957 .)

787t. A. T. Bharucha-Reid and L. W. Anderson: Ergodic projections for periodic Markov chains and processes.

This paper is concerned with the study of the ergodic properties of periodic Markov chains and processes. Let $P=\left(p_{i j}\right)$ denote the matrix of transition probabil- 
ities associated with a temporally homogeneous Markov chain with a denumerable set of states. The Markov matrices satisfy the relation $P^{m+n}=P^{m} P^{n}$, and form a semi-group of operators $\subseteq=\left\{P_{n}: n=0,1, \cdots\right\}$ on the Banach space $l$. We assume the Markov chain is periodic with period $\tilde{\omega}$; hence $P^{n+\tilde{\omega}}=P^{n}$. Let $\pi^{n}=n^{-1} \sum_{i-1}^{n} P^{i-1}$. As $n \rightarrow \infty \pi^{n}$ converges to the operator $\pi(\tilde{\omega})=\tilde{\omega}^{-1}(I+P+\cdots+P \bar{\omega}-1)$, with $\pi P=P \pi$ $=\pi$, and $\pi=\pi^{2}$. Now let $P(t)=\left(p_{i j}(t)\right)$ denote the matrix of transition probabilities associated with a temporally homogeneous Markov process with a denumerable set of states. The Markov matrices satisfy the relations $P(0)=I, P(s+t) P(s) P(t)$, and form a semi-group of operators $S=\{P(t): t \geqq 0\}$ on $l$ which has the properties $P(t) x \geqq 0$, and $\|P(t) x\|=\|x\|$ whenever $0 \leqq x \in l$, and $\|P(t) x-x\| \rightarrow 0$ as $t \downarrow 0$, for each $x \in l$. For periodic processes we have $P(t+\tilde{\omega})=P(t)$. Let $\pi(T)=T^{-1} \int_{0}^{T} P(t) d t$. As $T \rightarrow \infty, \pi(T)$ converges to the integral operator $\pi(\tilde{\omega})=\tilde{\omega}^{-1} \int_{0}^{\tilde{\omega}} P(t) d t$. Fixed point theorems are also obtained. Examples, and applications to some problems in applied stochastic processes are given. (Received July 11, 1957.)

\section{8t. I. B. Fleischer: The entropy of an infinite probability space.}

Let $f(t)$ be a nonincreasing function on the unit interval. Define the entropy of a finite probability space as the expectation of $f(p)$ and that of an infinite space $X$ as the supremum of entropies of the finite spaces obtained by restricting $p$ to a finite Borel subfield of $X$. Theorem: let $\{A\}$ be the atoms of the measure algebra of $X$ and $N$ be their complement. Suppose $f(t)$ is $O\left(t^{-1}\right)$ at zero. Then the entropy of $X$ is $\sum p(A) f(p(A))+p(N) \lim _{t \rightarrow 0+} f(t)$. In particular, Shannon's logarithmic entropy can be finite only for a purely atomic space. This work was partly done while the author was employed at the Bell Telephone Laboratories. (Received July 10,1957.)

\section{H. M. Gurk: Single-server, time-limited queues.}

Lindley (The Theory of Queues with a Single Server, Proc. Cambridge Phil. Soc. vol. 48 (1952) pp. 277-289) has solved the general case of a single server conservative queuing system with general arrival interval and service time distributions. His method provides the waiting time distribution of a customer (steady-state conditions assumed) by means of an integral equation. His results are extended here so as to cover the general case of time-limited queues, i.e., a customer will wait no more than a fixed time $T_{0}$. Assuming independent arrival intervals and service times, then if $A(t), S(s)$, and $W(x)$ are respectively the arrival interval, service time, and waiting time distribution functions, then for $0 \leqq x<T_{0}, W(x)=\int_{0}^{x}[S(x-t)$

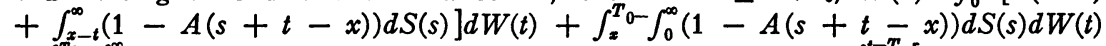
$+\int_{0}^{T_{0}-t} \int_{T_{0}-}^{\infty} I\left(s+t, T_{0}, x\right) d S(s) d W(t)$ where for $t>T_{0} I\left(t, T_{0}, x\right)=\int_{0}^{t-T_{0}} \cdot[1-A(t-\xi-x)$ $\left.+I\left(t-\xi, T_{0}, x\right)\right] d A(\xi)$. If $S(s)$ is an exponential distribution, then the last double integral in the equation for $W(x)$ can be replaced by $+\left[1-W\left(T_{0^{-}}\right)\right]$ - $\int_{0}^{\infty}\left(1-A\left(T_{0}+s-x\right)\right) d S(s)$. $W(x)$ has been determined for several examples of $S(s)$ and $A(t)$. In particular the Poisson arrival, exponential service case is given. (Received July $8,1957$.

\section{J. G. Kemeny and J. L. Snell (p): Semimartingales of Markov chains.}

An absorbing Markov chain is one that has a set of "boundary" states, where the process ends with probability 1 . There is given a finite absorbing Markov chain, and non-negative values assigned to the elements of the boundary. An upper semimartingale is a function on the states of the chain, such that the expected value of 
the function after one step from any state is greater than or equal to the value of the function at the state. A martingale is a semimartingale for which equality holds. A representation theorem is developed for all non-negative semimartingales with the prescribed boundary values in terms of martingales of modified chains. A modified chain is one obtained by adding interior states to the boundary, and assigning zero to these. The representation is unique and leads to a simple geometric interpretation. With the exception of certain degenerate cases, the non-negative upper semimartingales form a convex cubic polyhedron. In degenerate cases the polyhedron reduces to smaller polyhedra of the same dimension, including a simplex in the most degenerate case. Certain extensions and applications are discussed. In particular, the representation theorem yields a representation of discrete subharmonic functions in terms of harmonic functions. (Received May 22, 1957.)

791t. J. G. Kemeny and J. L. Snell: A fundamental matrix for regular Markov chains.

Let $P$ be transition matrix of a regular finite Markov chain. Let $L$ be the limit of $P^{n}$. The matrix $Z$ is defined as $(I-P+L)^{-1}$. Let $a_{j}$ be the limiting probability of being in state $j$. Let $s_{i j}^{n}$ be the mean number of times that the process is in state $j$, given that it starts in state $i$. It is shown that $s_{i j}^{n}-n a_{j}$ approaches a limit, and this limit is the $i j$-entry of $Z-I$. It is also shown that other basic quantities for the Markov chain can be expressed simply in terms of $Z$. For example, if $W$ is the matrix whose $i j$-entry is $z_{j j}$, and $D$ the diagonal matrix with $1 / a_{j}$ as $j j$-entry, then the mean number of steps to go from state $i$ to state $j$ is the $i j$-entry of $(I-Z+W) D$. A similar expression exists for the variance of this quantity. It is well known that the variance of the function giving the number of times that the process is in state $j$ in the first $n$ steps, normalized by $n^{1 / 2}$, approaches a limit independent of the starting state. It is shown that this limiting value is $a_{j}\left(2 z_{j j}-a_{j}-1\right)$. (Received June 26, 1957.)

792t. D. J. Newman and M. S. Klamkin: Expectations for sums of powers.

Numbers are chosen from the interval $(0,1)$ in a random unbiased way, and the process is continued until the sum of their $n$th powers exceeds 1 . The expected number of such choices is then determined. For $n=1$, the problem has been solved by $\mathrm{W}$. Weissblum in which case the answer is $e$. In this note we solve the general problem in terms of definite integrals and find, thereby, the asymptotic behavior as $n \rightarrow \infty$. Heuristically, it might be reasoned, the "average" number is $1 / 2$ and so the expected number should be around $2^{n}$. This is not the case and in fact it turns out that the expected number $E_{n}$ is asymptotic to $n e^{--\gamma}$. (Received July 9, 1957.)

\section{3t. Valdemars Punga: Random sampling applied to the solutions of systems of linear equations.}

The paper discusses essentially the Monte Carlo type method devised by J. von Neumann and S. M. Ulam for the inversion of matrices, but slightly modifying and simplifying the method applies it directly to the solutions of systems of linear equations of the type: $x_{i}=a_{i 1} x_{1}+a_{i 2} x_{2}+\cdots,+a_{i i-1} x_{i-1}+a_{i i+1} x_{i+1}+\cdots,+a_{i n} x_{n}+A_{i}$, $i=1,2, \cdots, n$, where coefficients $a_{i j}$ are positive and $a_{i 1}+a_{i 2}+\cdots,+a_{i i-1}+a_{i i+1}$ $+\cdots,+a_{i n}<1 . A_{i}$ can be any positive or negative numbers. (Received July 10, 1957.) 


\section{Edgar Reich: Asymptotic properties of queueing times when the} arrival epochs are arbitrary.

Given the sequences $\left\{s_{n}\right\}, s_{n} \geqq 0$ (the service periods), and $\left\{t_{n}\right\}, 0=t_{1} \leqq t_{2} \leqq \cdots$ (the arrival instants), one obtains the sequence of queueing times by the relations $q_{0}=0, q_{n+1}=\max \left(0, q_{n}+s_{n}+t_{n}-t_{n+1}\right)$. The prime problem is to find asymptotic results for $\left\{q_{n}\right\}$ in terms of $\left\{t_{n}\right\}$ and $\left\{s_{n}\right\}$. Classisal results of Lindley, and KieferWolfowitz deal with the case where $\left\{t_{n+1}-t_{n}\right\},\left\{s_{n}\right\}$ are independent sequences of mutually independent equidistributed random variables. We study the case where the classical assumptions are relaxed, and $\left\{t_{n}\right\}$ and $\left\{s_{n}\right\}$ are arbitrary sequences of real numbers, for which only certain averages exist. The results yield additional information in the classical case, and may also be applied to situations where the classical assumptions are violated, e.g., when $\left\{t_{n}\right\}$ are the events of a Poisson process of variable density. Here one finds conditions under which (i) $E q_{n}=O(1)$, and (ii) $q_{n}=O(n)$ w. p. 1. (Received June 14, 1957.)

795. J. M. Shapiro: Sums of powers of independent random variables.

For $n=1,2, \cdots$, let $S_{n}=x_{n 1}+\cdots+x_{n k_{n}}-A_{n}$ be the sum of independent infinitesimal random variables minus suitably chosen constants $A_{n}$ and let $F_{n}(x)$ be the distribution function of $S_{n}$. For $r>1$ let $S_{n}^{r}=\left|x_{n 1}\right| r+\cdots+\left|x_{n k_{n}}\right| r-B_{n}(r)$ where $B_{n}(r)$ are suitably chosen constants and let $F_{n}^{*}(x)$ be the distribution function of $S_{n}^{r}$. Let $F(x), F r(x)$ and $H(x)$ be distribution functions. Suppose $\left(^{*}\right) \lim _{n \rightarrow \infty} F_{n}^{*}(x)=\operatorname{Fr}(x)$ and $\lim _{r \rightarrow \infty} \operatorname{Fr}^{r}(x)=H(x)$. Then it is shown that $H(x)$ is the distribution function of the sum of two independent random variables, one Gaussian and the other Poisson (including their degenerate cases). Other results are obtained. In particular let $F_{n}(x) \rightarrow F(x)$ and let $G(x)$ be the nondecreasing function determined by the LévyKhintchine formula for the characteristic function of (the infinitely divisible) distribution $F(x)$. Suppose $G(x)$ is nonincreasing for $x<-1$ and $x>1$. Then if either (1) the random variables $\left(x_{n k}\right)$ are symmetric, $(2)\left(x_{n k}\right)$ are positive random variables and $A_{n}=0$, or (3) for each $n, x_{n 1}, \cdots, x_{n k_{n}}$ are identically distributed and $A_{n}=0$, it follows that for suitably chosen constants $B_{n}(r),\left(^{*}\right)$ holds where $H(x)$ is a Poisson distribution (or an improper distribution). (Received July 8, 1957.)

\section{ToPOLOGY}

796t. F. W. Anderson and R. L. Blair: Characterizations of certain lattices of functions.

Let $K$ be a chain in its interval topology and let $X$ be a compact Hausdorff space. A sublattice $L$ of $C(X, K)$, the lattice of all $K$-valued continuous functions on $X$, is characterizing in case whenever $x \neq y$ in $X$ and $f, g \in L$, there is an $h \in L$ such that $h(x)>f(x)$ and $h(y)<g(y)$. If $K$ is conditionally complete and dense-in-itself, then a sublattice $L$ of $C(X, K)$ is a $Q$-sublattice in case $L$ contains a set $Q$ of constant functions order-dense in $K$. Necessary and sufficient conditions are given in order that an abstract lattice be lattice-homomorphic to a characterizing $Q$-sublattice of $C(X, K)$ for some $X$ and $K$. Also given are necessary and sufficient conditions that this homomorphism be an isomorphism. With certain further restrictions on $X$ and $K$ a characterization of $C(X, K)$ itself is obtained. In particular, a new solution of Birkhoff's Problem 81 [Lattice theory, rev. ed., 1948] is given. (Received July 11, 1956.) 


\section{Steve Armentrout: A study of certain plane-like spaces without the use of arcs.}

In this paper, a space $T$ satisfying certain axioms, denoted by 0, I, II, III, IV, and $\mathrm{V}$, is studied, and it is shown that certain propositions which hold in a space satisfying axioms 0 and 1-5 of R. L. Moore's Foundations of point set theory also hold in the space $T$. No argument in the development makes use of an arc. Axioms 0 , I, and III are axioms $0,1^{\prime}(4)$ [Axiom $1^{\prime}$ with condition (5) omitted], and 3, respectively, of Foundations, and Axiom II is the following: If $P$ is a point of a region $R$, there is a connected domain $D$, lying in $R$ and containing $P$, such that if $X$ is a point of $D$, there is a compact continuum lying in $R$ and containing $P$ and $X$. A closed chain of compact continua is a certain finite collection of compact continua; a $J$-set is a continuum which is the sum of the sets of such a collection. The $J$-sets are, in a certain sense, substitutes for simple closed curves. Axioms IV and V are statements about $J$-sets and their complementary domains, analogous to axioms 4 and 5 of Foundations. (Received July 5, 1957.)

\section{8t. Joseph Auslander: Mean-L-stable systems.}

Let $X$ be a compact metric space with metric $\rho$ and let $T$ be a self-homeomorphism of $X$. The system $(X, T)$ is called mean- $L$-stable provided that for every $\epsilon>0$ there exists $\delta>0$ such that $\rho(x, y)<\delta$ implies $\rho\left(x T^{n}, y T^{n}\right)<\epsilon$ for all $n$ except a set of upper density less than $\epsilon$. If $\delta$ can be chosen so that $E$ is vacuous, $(X, T)$ is called uniformly- $L$-stable. The points $x$ and $y$ are called proximal if $\inf _{n} \rho\left(x T^{n}, y T^{n}\right)=0$; otherwise they are called distal. It is shown that the set of distinct proximal pairs in a mean- $L$-stable system has invariant measure zero in $X \times X$. "Proximal" is an equivalence relation, and the quotient system is shown to be uniformly- $L$-stable. A minimal mean- $L$-stable system containing a point distal to all other points is locally almost periodic. The following decomposition theorem is obtained: $X=\mathrm{U}_{\alpha} N_{\alpha}$, where each $N_{\alpha}$ is closed invariant and contains precisely one minimal set $M_{\alpha}$. Every equivalence class of mutually proximal points is represented in just one $M_{\alpha}$, and $\mathrm{U}_{\alpha} M_{\alpha}$ has invariant measure one. (Received July $15,1957$. )

799. W. D. Barcus and J. P. Meyer (p): On the suspension of the loop space. Preliminary report.

Let $X$ be a 1-connected topological space, $\Omega$ the space of loops on $X, S \Omega$ the suspension of $\Omega$. Define a map $p: S \Omega \rightarrow X$ by $p(t, \omega)=\omega(t), t \in I, \omega \in \Omega ; p$ is dual to the inclusion $i$ : $X \subset \Omega S X$ used to study the homotopy suspension. Transform $p$ into a fibre map by the standard method. Then it can be shown that the fibre has the (weak) homotopy type of the join $\Omega * \Omega$. The homology sequence of the fibering is closely related to G. W. Whitehead's sequence containing the homology suspension (Ann. of Math. vol. 62 (1955) p. 254), but has an extra term. One application is the following corollary, which extends Whitehead's result by one dimension: $A$ cohomology operation of type $(n, q ; \pi, G), q \leqq 3 n$, is additive if and only if it is a suspension. (Received July $12,1957$.

\section{R. H. Bing: Conditions under which a surface in $E^{3}$ is tame.}

Suppose $X_{0}, X_{1}, X_{2}, \cdots$ is a sequence of homeomorphic sets in a metric space. Then $X_{1}, X_{2}, \cdots$ is said to converge homeomorphically to $X_{0}$ if there is a sequence of numbers $\epsilon_{1}, \epsilon_{2}, \cdots$ converging to 0 and a sequence of homeomorphisms $h_{1}, h_{2}, \cdots$ such that $h_{i}$ takes $X_{i}$ onto $X_{0}$ and moves no point by more than $\epsilon_{i}$. Suppose $S$ is a 
continuum (closed connected set) in $E^{3}$ which is a 2-manifold (locally topologically like $E^{2}$ ). Then $E^{3}-S$ has two components $D_{1}, D_{2}$. It is shown that $S$ is tame (there is a homeomorphism of $E^{3}$ onto itself taking $S$ onto a polyhedron) if there is a sequence of 2-manifolds in $D_{1}$ converging homeomorphically to $S$ and a sequence of 2-manifolds in $D_{2}$ converging homeomorphically to $S$. (Received July 9, 1957.)

801t. R. H. Bing: $E^{3}$ does not contain uncountably many mutually exclusive wild surfaces.

A surface $S$ (closed set which is a 2-manifold) is wild in $E^{3}$ if there is no homeomorphism of $E^{3}$ onto itself that takes $S$ onto a polyhedron (sum of a locally finite collection of simplexes). It is shown that if $G$ is an uncountable collection of mutually exclusive surfaces in $E^{3}$, then not more than a countable number of elements of $G$ are wild. This is proved as follows: If an element of $G$ is wild, this element has a component that is wild because locally tame sets are tame. Hence if $G$ contains uncountably many wild elements, there exists an uncountable collection $G^{\prime}$ of mutually exclusive wild connected surfaces. Some element of $G^{\prime}$ would contain sequences converging homeomorphically to it from both sides. But a surface is tame if there are sequences converging to it homeomorphically from both sides. (Received July 9, 1957.)

\section{2t. William Browder: Homology of loops of certain H-spaces.}

Let $X$ be a simply connected $H$-space whose Pontrjagin ring $H_{*}(X)$ is an exterior algebra on odd dimensional generators $x_{1}, x_{2}, \cdots$, where $\operatorname{dim} x_{i} \leqq \operatorname{dim} x_{i+1}$ and the number of $x_{i}$ 's such that $\operatorname{dim} x_{i}<n$ is finite for any $n$, (coefficients will always be assumed to be an integral domain or a field). Then in the spectral sequence of homology for the space of paths of $X, E^{n}$ is an anti-commutative algebra (Hopf algebra for field coefficients) for $n \geqq 2$, in particular $E^{2}=H_{*}(\Omega) \otimes H_{*}(X)$ (see J. C. Moore, Boletin de la Sociedad Matematica Mexicana vol. 1 (1956) pp. 28-37). Theorem: $H_{*}(\Omega)$ is a polynomial ring generated by $y_{1}, y_{2}, \cdots$, with the property that $\sigma\left(y_{i}\right)=x_{i}$, where $\sigma$ is the homology suspension associated with an acyclic fibre space. The theorem is first proved for fields, using the structure theorem of Borel for Hopf algebras over perfect fields (A. Borel, Ann. of Math. vol. 57 (1953) pp. 115-206). The result is then extended to integral domains using the homomorphism induced by the canonical homomorphism of the integral domain into its quotient field. (Received July 8, 1957.)

803t. Morton Brown: Continuous collections of higher-dimensional hereditarily indecomposable continua.

R. H. Bing has shown Trans. Amer. Math. Soc. vol. 71 (1951) pp. 267-273 that there exists in Euclidean $N$-space an $N-1$ dimensional hereditarily indecomposable continuum with exactly two complementary domains which separates Euclidean $\mathrm{N}$-space irreducibly. The author shows that Euclidean $\mathrm{N}$-space with the origin deleted is the sum of a continuous collection of such continua. (Received July 15, 1957.)

\section{C. E. Burgess: Chainable continua and indecomposability.}

Let $M$ denote a compact connected metric space. If for each positive number ${ }^{\epsilon}$ there exists a finite sequence of open sets $D_{1}, D_{2}, \cdots, D_{n}$ covering $M$ such that each $D_{i}$ has a diameter less than $\epsilon$ and $D_{i} \cdot D_{j} \neq 0$ only if $|i-j|<2$, then $M$ is said to be linearly chainable. If this is modified so that $D_{1} \cdot D_{n} \neq 0$, then $M$ is said to be circularly 
chainable. In order that $M$ should be either indecomposable or 2-indecomposable, it is sufficient that any one of the following four conditions be satisfied: (1) $M$ is both circularly chainable and unicoherent; (2) $M$ is circularly chainable and is irreducible between some two points; (3) $M$ is circularly chainable and is not multicoherent; (4) every proper subcontinuum of $M$ is circularly chainable. If $M$ is linearly chainable, then in order that $M$ should be circularly chainable, it is necessary and sufficient that $M$ should be either indecomposable or 2-indecomposable. (Received July 10,1957.)

805. Eugenio Calabi: Fibre bundles techniques in the modification of complex analytic structure. Preliminary report.

Let $\operatorname{Gr}(p, q ; C)$ denote the complex Grassmann manifold of $p$-dimensional linear subspaces $V_{p}$ of a $(p+q)$-dimensional vector space $V_{p+q}$ over the complex field $C$. The complement of a $\operatorname{Gr}(n+1, n-1 ; C)$ in $\operatorname{Gr}(n+1, n ; C)(n=1,2, \cdots)$ is naturally a fibre bundle over $\operatorname{Gr}(n, n ; C)$ with a complex affine $n$-space $A_{n}$ as fibre, and the group $V_{n}$ of translations of $A_{n}$ as structure group. The restriction $B_{n}$ of this bundle to a suitable open, everywhere dense submanifold $H_{n}$ of $\operatorname{Gr}(n, n ; C)$ is a trivial bundle in terms of its real analytic structure, but not in terms of its complex one. In fact, if $\Lambda$ is a subgroup of $V_{n}$ generated by $2 n$ real-linearly independent translations, then $\Lambda$ acts on $B_{n}$. Factoring $\Lambda$ out, one obtains a $2 n$-torus bundle over $H_{n}$; the restriction of the latter to a compact, complex submanifold $K_{n}$ of $H_{n}$ for $n \geqq 2$ yields a compact, complex manifold whose Chern classes are distinct from those of the product of $K_{n}$ by a complex $2 n$-toral group. The complex structure of other complex vector- and toral bundles can be modified by similar techniques. (Received July 10,1957.)

806. M. L. Curtis: A note on Kosinski's r-spaces.

Following Kosihski (Fund. Math. vol. 43 (1955) 111-124) we call a point $x$ in a space $X$ an $r$-point if $x$ has arbitıarily-small neighborhoods $U$ such that for each $y \in U$ there is a deformation retraction of $\bar{U}-y$ onto $\bar{U}-U$. A space $X$ is an $r$-space if it is finite dimensional, compact metric and each point is an $r$-point. Problem 7 in Kosinski's paper asks if $(a, b) \in A \times B$ being an r-point of $A \times B$ implies that $a$ and $b$ are $r$-points of $A$ and $B$ respectively. We answer this question in the negative by giving a 4-dimensional finite polyhedron $P^{4}$ which is not an $r$-space but is such that its cartesian product $P^{4} \times S^{1}$ with a circle $S^{1}$ is an r-space. It is not known if $P^{4} \times S^{1}$ $i^{\text {s a topological manifold. (Received July } 5,1957 \text {.) }}$

807. M. R. Demers and Herbert Federer (p): On 2 dimensional Lebesgue area. II.

Suppose $f$ is any continuous map of a 2 dimensional finitely triangulable space into Euclidean $n$-space. It is proved that the Lebesgue area of $f$ does not exceed the sum of the Lebesgue areas of the $C_{n, 2}$ principal projections of $f$ into 2 -space. The argument uses a new result concerning the local structure of monotone images of finitely triangulable spaces, and a general theorem connecting the stable values of a map into $k$-space with the stable values of the sections of this map corresponding to a fibering of $k$-space into parallel $k-1$ dimensional planes. (Received May 21, 1957.)

\section{8t. Edward Fadell: On extending a theorem to Serre fiber spaces.}

The objective of this paper is to extend the following theorem (Hurewicz-Fadell, Proc. Nat. Acad. Sci. vol. 43 (1957) pp. 241-245) to fiber spaces in the sense of Serre. Let $\mathfrak{F}=\{X, B, p\}$ denote a fiber space with fiber $F$ such that $B$ is $(r-1)$-connected, $r>1$. Then in the associated spectral sequence $\left\{E_{j}, d_{j}\right\}, E_{j}=H(B, H(F))$ for 
$2 \leqq j \leqq r, d_{j}=0$ for $2 \leqq j \leqq r-1$, and the differential operator $d_{r}$ is given by a cap product $d_{r}(h)=\gamma \cap h, h \in H(B, H(F))$, where $\gamma$ is the characteristic cohomology class of $B$ and $\pi_{r}(B)$ and $H(F)$ are suitably paired. In the above cited reference crucial use was made of continuous maps $\tilde{\lambda}: \widetilde{B} \times F \rightarrow X(\widetilde{B}$ is the space of paths starting at the base point in $B$ ) and $\bar{\lambda}: X^{I} \rightarrow X^{I}$ induced by a lifting function $\lambda$ for $\mathcal{F}$. For Serre fiber spaces, lifting functions are no longer available and the extension of the above theorem requires the use of the Covering Homotopy Theorem to define, directly at the singular chain level, chain mappings analogous to those induced by the continuous maps $\bar{\lambda}$ and $\bar{\lambda}$. (Received June 14, 1957.)

809t. R. H. Fox and J. W. Milnor: Singularities of 2-spheres in 4space and equivalence of knots.

The boundary $\dot{\sigma}$ of a small 4 -simplex $\sigma$ around a point $x$ of an oriented polyhedral surface $F$ in oriented 4 -space will intersect $F$ in an oriented simple closed curve $C$. If $C$ is knotted in the 3-sphere $\dot{\sigma}$ then $x$ is a singular point and the knot type $k$ of $C$ is the singularity at $x$. Let $k^{-1}$ denote the knot type obtained from $k$ by reversing the orientation of $C$ and taking its mirror image. Define $k$ and $l$ to be equivalent if there exists a polyhedral 2-sphere in 4-space having only two singular points, one of type $k$ and one of type $l^{-1}$. Then the equivalence classes of knots form an abelian group $G$ under the usual product operation. A collection $k_{1}, \cdots, k_{n}$ of knot types occurs as the collection of singularities of some 2 -sphere iff the product $k_{1} \cdots k_{n}$ is equivalent to the trivial knot. In order that $k$ and $l$ be equivalent it is necessary that the product of their Alexander polynomials have the form $a(t) a(1 / t)$ for some integral polynomial $a(t)$. Consequently $G$ is not finitely generated. $G$ contains elements of order 2 ; it is conjectured that $G$ also contains elements of order $>2$. (Received July 15, 1957.)

810. E. E. Grace: The existence of cut sets in totally nonaposyndetic continua.

Results of F. Burton Jones (Amer. J. Math. vol. 70 (1948) pp. 403-413) on the existence of weak cut points in certain nonaposyndetic compact metric continua are extended to a class of connected topological spaces which have a certain generalized completeness property. Stronger cutting properties are obtained and the use of an extension of the idea of nonaposyndesis yields weak cut sets in some cases where there are no cut points. (Received July 8, 1957.)

\section{1t. Edward Halpern: On the cohomology of certain loop spoces.}

Let $J$ be a principal ideal domain. A monogenic twisted polynomial $J$-algebra of binomial type and height $h,(2 \leqq h \leqq \infty)$, is free $J$-module generated by a sequence of elements $x_{0}, x_{1}, \cdots, x_{h-1}$ and multiplication defined by $x_{m} x_{n}=(m, n) x_{m+n}$ for $m+n<h,((m, n)$ is the binomial coefficient $(m+n !) /(m ! n !))$, and $x_{m} x_{n}=0$ for $m+n \geqq h$; it is denoted by $J\left[x_{1} ; h,(m, n)\right]$. Let $X$ be a topological space with (singular) cohomology $H^{*}(X, J)=J[x] /\left(x^{h}\right), 2 \leqq h \leqq \infty$, with $x$ of even degree $d$. Let $\Omega$ denote the space of loops at a base point of $X$. (a) If $J$ has characteristic zero then $H^{*}(\Omega, J)$ $\cong \wedge J\left(z_{1}\right) \otimes J\left[z_{2} ; \infty,(m, n)\right]$, where $z_{1}, z_{2} \in H^{*}(\Omega, J)$ have degrees $d-1$ and $h d-2$ respectively. (b) If $J$ has characteristic $p \neq 0$ then $H^{*}(\Omega, J) \cong \wedge J\left(z_{1}\right) \otimes_{i \geq 0} J\left[z_{2 p^{i}} ; p,(m, n)\right]$, where $z_{1}, z_{2 p^{i}} \in H^{*}(\Omega, J)$ have degrees $d-1$ and $p^{i}(h d-2)$ respectively, $\left(\otimes_{i \gtrless 0}\right.$ denotes the "weak" tensor product). In particular, the theorem applies to complex and quaternionic projective $n$-spaces $(1 \leqq n \leqq \infty)$ and the Cayley plane. (Received June 7, 1957.) 
812. Melvin Henriksen (p) and J. R. Isbell: Some properties of compactifications. I.

A compactification of a space $X$ is a compact space containing a dense subspace homeomorphic with $X$. (All spaces mentioned herein are assumed to be completely regular.) We are concerned with those properties $P$ of spaces such that for all spaces $X\left(^{*}\right)$ if the complement of $X$ in one of its compactifications has $P$, then the complement of $X$ in any of its compactifications has $P$. These include compactness, local compactness, $\sigma$-compactness, countable compactness, the Lindelöf property, paracompactness, and countable paracompactness. A continuous onto mapping $f: X \rightarrow Y$ that has a continuous extension over some compactification $A X$ of $X$ onto some compactification $B Y$ of $Y$ that maps $A X-X$ homeomorphically onto $B Y-Y$ is called a meshing map. A closed continuous onto mapping $f: X \rightarrow Y$ such that for each $y \in Y, f^{-1}(y)$ is compact is called a fitting map. A property $P$ such that whenever $f: X \rightarrow Y$ is a meshing (resp. fitting) map, then $X$ has $P$ iff $Y$ has $P$ is called a meshing (resp. fitting), property. $\mathcal{P}$ satisfies $\left({ }^{*}\right)$ iff it is a meshing property. Every meshing map (resp. fitting property) is a fitting map (resp. meshing property), but not conversely. All of the properties listed above are actually fitting properties. (Received July 5, 1957.)

813t. Melvin Henriksen and J. R. Isbell: Some properties of compactifications. II.

(Terminology is as in the preceding abstract.) $\beta X$ denotes the Stone-Cech compactification of the space $X$. A space $X$ is said to have property $\odot$ at infinity if $\beta X-X$ has property $P$. If $P$ is a meshing property, then so is $P$ at infinity. $P$ is a fitting property iff $P$ at infinity is. Compactness at infinity is just local compactness. $X$ is locally compact at infinity iff the set $R(X)$ of all points of $X$ at which $X$ fails to be locally compact is compact. In general, for any meshing property $P, X$ has property $\mathcal{P}$ at infinity iff $R(X)$ has property $\odot . X$ is $\sigma$-compact at infinity iff $X$ is an absolute $G_{\delta} . X$ is Lindelöf at infinity iff every compact subset of $X$ is contained in a compact set of countable character. Thus every metrizable space is Lindelöf at infinity. A countable product of spaces $\sigma$-compact (resp. Lindelöf) at infinity is $\sigma$-compact (resp. Lindelöf) at infinity. (Received July 5, 1957.)

\section{4t. J. G. Horne, Jr.: On $O_{\omega}$-ideals in $C(X)$.}

Let $S$ be a set with relation $R$. An $R$-ideal is a subset $J \subset S$ satisfying (1) $f \in J$, $h \in S$ and $h R f$ imply $h \in J$; (2) $f_{1}, f_{2} \in J$ imply there exists $e \in J$ such that $f_{i} R e$ for $i=1,2$. The family of all proper $R$-ideals admits the dual Stone topology. Now suppose $S$ is a commutative semigroup with identity. In previous notes (see Bull. Amer. Math. Soc. Abstract 63-1-140) we have considered $O$-ideals where $O=\{(f, e): f e=f\}$. In this note we consider $O_{\omega}$-ideals where $O_{\omega}=\{(f, e): f \in e S\}$. If $S$ is the multiplicative semigroup of a ring with identity then the $O_{\omega}$-ideals are precisely the ring ideals which are $O_{\omega}$-directed. Let $X$ be a topological space and let $C(X)$ denote the ring of real-valued continuous functions on $X$. THEOREM 1. If $J \subset C(X)$ is an ideal such that $f \in J$ implies $|f|^{1 / 2} \in J$, then $J$ is an $O_{\omega}$-ideal. COR. 1. The prime ideals and the m-closed ideals of $C(X)$ are $O_{\omega}$-ideals. COR. 2. The image under a multiplicative isomorphism of a prime or m-closed ideal is ditto. THEOREM 2. Every ideal of $C(X)$ is an $O_{\omega}$-ideal iff $x \in X$ and $f \in C(X)$ imply there is a neighborhood $U$ of $x$ such that either $f \mid U \geqq 0$ or $f \mid U \leqq 0$. (See Bull. Amer. Math. Soc. Abstracts 60-4-462 and 61-2-183 of Gillman and Henriksen). (Received July 8, 1957.) 


\section{5t. J. G. Horne, Jr.: On primary ideals in $C(X)$.}

We use the notation of the previous abstract. A primary ideal is an ideal which is contained in at most one maximal ideal. For $N C C(X)$ let $L(N)=\{f: f e=f$ for some $e \in N\}$. The collection of maximal ideals of $C(X)$ is denoted by $\mathfrak{R}$, the topological space consisting of $\mathfrak{N}$ and the dual Stone topology by $\mathfrak{N}_{\Gamma}$ (see above abstract). An ideal $P$ is prime-like if the conditions $f \notin P, f e=f$ and $k e \in P$ imply $k \in P$. If $N$ is a prime-like ideal therı $L(N)$ is a maximal $O$-ideal. Gillman and Henriksen proved that the maximal $O$-ideals and the prime ideals of $C(X)$ are primary (Trans. Amer. Math. Soc. vol. 77 (1956) p. 342). THEOREM 1. For an ideal $P \subset C(X)$ these are equivalent: (1) $P$ contains a maximal $O$-ideal; (2) $P$ is a primary ideal; (3) $P$ is a prime-like ideal. An ideal $N \subset C(X)$ is $f x e d$ if there exists $x \in X$ such that $f(x)=0$ for all $f \in N$. Suppose $X$ is a completely regular $G_{\delta}$-space. THEOREM 2 . The following conditions are equivalent for a maximal ideal $N \subset C(X):$ (1) $N$ is fixed; (2) $N$ is the union of its principal primary ideals; (3) $N$ is an isolated point of $\mathfrak{N}_{\Gamma} ;(4) L(N)$ is contained in a proper principal ideal. $N$ is bounded by $f$ in the sense of Pursell (Pacific J. Math. vol. 5 (1955) p. 964) iff the principal ideal $[f]$ contains $L(N)$. (Received July $8,1957$. )

\section{W. E. Malbon: Invariants for quasi-compact mappings.}

A study is made of topological properties which are invariant under quasi-compact mappings where the image space is $T_{1}$ and has properties $M$ and/or $E$. A $T_{1}$ space has property $M$ provided every sequence converges to at most one point. A $T_{1}$ space has property $E$ provided for any limit point $x$ of a set $K$ there exists a sequence $S$ of infinitely many distinct points of $K$ such that $S$ converges to $x$. While Hausdorff spaces have property $M$ and weakly separable spaces property $E$, bicompact $T_{1}$ spaces with properties $M$ and $E$ exist which are neither weakly separable nor Hausdorff. Examples of the results are: (1) for any compact metric space $A$ each associated decomposition space $A^{*}$ is compact and metric if and only if $A^{*}$ has property $M$; (2) for any open (quasi-compact with compact, connected point inverses) mapping $f(A)=B$, the property of being locally compact, separable and metric is invariant if and only if $B$ has property $M$; (3) for $f(A)=B, A$ having properties $M$ and $E$ and $B$ property $E, f$ compact (in the limit point sense) is equivalent to $f$ closed and $f^{-1}(b)$, $b$ in $B$, compact (in the limit point sense). (Received July 3,1957 .)

\section{Frank Raymond: Duality theorem for homology manifolds.}

Let $X$ be a compact, connected, finite dimensional, Hausdorff space. Let $H_{p}$ denote the $p$ th singular homology with coefficients in a group or field, $G$. If (1) for each $x$ in $X, H_{p}(X, X-x)=0, p \neq n$, and $=G$, for $p=n$, (2) for each $x$ in $X$, $H_{n}(X) \rightarrow H_{n}(X, X-x) \rightarrow 0$ exact, (3) for each proper closed subset $F$ of $X$, the injection of $H_{n}(F)$ into $H_{n}(X)$ is 0 , then there exists an isomorphism of the Cech cohomology sequence, coefficients in $G, A$ closed, $\rightarrow H^{n-p}(X, A) \rightarrow H^{n-p}(X) \rightarrow H^{n-p}(A)$ $\rightarrow H^{n-p+1}(X, A) \rightarrow$ onto the singular homology sequence, coefficients in $G \rightarrow H_{p}(X-A)$ $\rightarrow H_{p}(X) \rightarrow H^{p}(X, X-A) \rightarrow H_{p-1}(X-A) \rightarrow$ and the corresponding vertical diagram is commutative. This is essentially $H$. Cartan's duality theorem. It follows that: $X$ is a Peano space, has the invariance of domain property, and interior and frontier points are characterized by dimension. $X$ is a Cantor manifold, if $n$-dimensional. If $X$ is $n$-dimensional, $l c_{1}^{n-1}$, field coefficients, then $X$ is an orientable $n$ - $\mathrm{gcm}$ in the sense of Wilder. If $X$ is an $L C_{1}^{n}$ (homotopy) orientable metric $n$-gcm (Wilder), then $X$ is a homology manifold in the above sense. The nonorientable case can also be treated. A similar duality theorem can be obtained using Cech homology theory. 
This work was sponsored in part by Air Force Contract No. A.F. 49(638)-104. (Received July 12, 1957.)

\section{J. H. Roberts: Arcs of homeomorphisms.}

Suppose $X$ is compact, metric, and of finite dimension $\leqq n$. For each positive integer $k$ let $H_{k}$ be the space of all homeomorphisms of $X$ into $I_{k}$, the $k$-dimensional interval. The topology for $H_{k}$ is the metric topology given by the formula: $d(f, g)$ $=\max d(f(x), g(x)), x X$. Theorem: If $k \geqq 2 n+2$ then $H_{k}$ is arc-wise connected, both locally and globally. Simple examples show that the theorem is false for $k<2 n+2$. (Received July 10, 1957.)

\section{R. H. Rosen: On tree-like continua and irreducibility.}

The following theorems, which are generalizations of theorems proved by $R$. $H$. Bing for snake-like continua, are proved. I. Every monotone upper-semi-continuous decomposition of a compact metric tree-like continuum gives a compact metric treelike continuum in the hyper-space. Definition: A tree-like continuum $M$ is said to be $k$-branched if for each $\epsilon$ greater than $0, M$ can be covered by an $\epsilon$-tree-chain with exactly $k$ end links and $k$ is the least such integer. II. Every compact metric tree-like continuum which is $k$-branched is irreducible about some set of $k$ points. In order to prove II. the following result is used: III. If $M$ is a compact metric nondegenerate continuum such that $M$ contains a subcontinuum $K$ such that $M$ is not the sum of two proper subcontinua both of which intersect $K$, then there is a point $q$ so that for any point $p$ in $K, M$ is irreducible from $p$ to $q$. (Received May 22,1957.)

\section{0t. Walter Rudin: Perfect subsets of compact spaces.}

If a compact Hausdorff space $X$ contains a perfect subset $P$ (i.e., $P$ is compact, nonempty, and has no isolated point), then $X$ contains a separable perfect set. (Equivalently, $X$ contains a countable set which is dense in itself.) PROOF:There exists a continuous real function $f$ on $X$ which maps $P$ onto the unit interval $I$, and there exists a compact subset $E$ of $P$, minimal with respect to the property that $f(E)=I$. This $E$ is perfect. For every rational $r \in I$, choose one $x_{r} \in E$ for which $f\left(x_{r}\right)=r$, and let $S$ be the set of all $x_{r}$ so chosen. Then $S$ is countable, the minimality of $E$ implies that $E$ is the closure of $S$, so that $E$ is separable. REMARK: Nevertheless, the set of all $x \in E$ such that $f(x)=t$ may have $2^{c}$ points, for every $t \in I$, where $c$ denotes the power of the continuum. This happens, for instance, when $X$ is the Čech compactification of a countable discrete space. (Received June 12,1957.)

821. W. L. Strother (p) and L. E. Ward, Jr.: Fixed points from neighborhood retracts.

The space of subsets of an absolute retract is known to be an absolute retract and hence to have the fixed point property. This note establishes that the space of subsets of a connected neighborhood retract has the fixed point property. Whether this space of subsets is an absolute retract remains an open question. (Received July 3, 1957.)

822t. L. E. Ward, Jr.: A fixed point theorem for multi-valued functions.

A continuum (=compact connected Hausdorff space) is said to be topologically chained if each pair of distinct points is contained in a subcontinuum with exactly two 
non-cutpoints. The topologically chained and hereditarily unicoherent continua are characterized in terms of their inherent order properties. Such continua contain the generalized trees (and hence the dendrites) as a subclass. Using their order properties it is shown that each topologically chained, hereditarily unicoherent continuum has the fixed point property for continuous multi-valued functions. This is a type of Scherrer fixed point theorem which extends recent results of Wallace, Capel and Strother, Borsuk, Plunkett, and the author. (Received July 10, 1957.)

823. R. F. Williams: Lebesgue area zero, dimension, zero, and finecyclic elements.

If $f: X \rightarrow E_{n}$ is a map where $X$ is compact, metric and 2-dimensional, then $f$ can be uniformly approximated by "quasi-linear" maps $\phi=g h$, where $h$ is a barycentric $\alpha$-map of $X$ onto a realized nerve $\alpha(X)$, and $g: \alpha(X) \rightarrow E_{n}$. Thus Lebesgue area, $L(f, X)$, can be defined. Theorem: $L(f, X)=0$ if and only if, $f$ can be uniformly approximated by maps $f^{\prime}$ such that either (1) $f^{\prime}(X)$ is the sum of a finite number of line intervals, (2) $f^{\prime}$ can be factored through a space of dimension $\leqq 1$, or (3) $f^{\prime}$ is quasi-linear and the elementary area, $a(f, X)$, is zero. Theorem: if $X$ is a continuous curve of finite degree of multi-coherence, then $L(f, X)=0$ if and only if the middle space of the monotone, light factorization of $f$, is of dimension $\leqq 1$, or, equivantly, has no finecyclic element. This last generalizes a theorem of T. Rado, Length and area, p. 467. For definitions and properties of fine-cyclic elements, see L. Cesari [Bull. Amer. Math. Soc. Abstract 61-6-698] and C. Neugebauer [Bull. Amer. Math. Soc. Abstract 63-2-340]. (Received June 21, 1957.)

\section{C. T. Yang: On maps of spheres into euclidean spaces.}

In this note it is proved that given any map of an $(n+1)$-sphere into the euclidean $n$-space there exist three points of the sphere which are mapped into a single point and such that the spherical distance between any two of these points is equal to a preassigned number $d, 0<d \leqq 2 \pi / 3$. This result includes a theorem of Kakutani (Ann. of Math., 1942) as a special case. Moreover, this result can be generalized just as the well-known Borsuk-Ulam's theorem generalized by Hopf (Portugaliae Math., 1944). (Received July 10, 1957.) 